\title{
Universality of the Hall conductivity in Interacting electron systems
}

Giuliani, Alessandro ; Mastropietro, Vieri ; Porta, Marcello

\begin{abstract}
We prove the quantization of the Hall conductivity for general weakly interacting gapped fermionic systems on two-dimensional periodic lattices. The proof is based on fermionic cluster expansion techniques combined with lattice Ward identities, and on a reconstruction theorem that allows us to compute the Kubo conductivity as the analytic continuation of its imaginary time counterpart.
\end{abstract}

DOI: https://doi.org/10.1007/s00220-016-2714-8

Posted at the Zurich Open Repository and Archive, University of Zurich

ZORA URL: https://doi.org/10.5167/uzh-128376

Journal Article

Accepted Version

Originally published at:

Giuliani, Alessandro; Mastropietro, Vieri; Porta, Marcello (2017). Universality of the Hall conductivity in Interacting electron systems. Communications in Mathematical Physics, 349(3):1107-1161.

DOI: https://doi.org/10.1007/s00220-016-2714-8 


\title{
Universality of the Hall conductivity in interacting electron systems
}

\author{
Alessandro Giuliani \\ University of Roma Tre, Department of Mathematics and Physics, \\ L.go S. L. Murialdo 1, 00146 Roma, Italy \\ Vieri Mastropietro \\ University of Milano, Department of Mathematics "F. Enriquez", \\ Via C. Saldini 50, 20133 Milano, Italy \\ Marcello Porta \\ University of Zürich, Mathematics Department, \\ Winterthurerstrasse 190, 8057 Zürich, Switzerland
}

\begin{abstract}
We prove the quantization of the Hall conductivity for general weakly interacting gapped fermionic systems on two-dimensional periodic lattices. The proof is based on fermionic cluster expansion techniques combined with lattice Ward identities, and on a reconstruction theorem that allows us to compute the Kubo conductivity as the analytic continuation of its imaginary time counterpart.
\end{abstract}

\section{INTRODUCTION}

Two-dimensional condensed matter systems often present remarkable transport properties. A famous example is the Integer Quantum Hall Effect (IQHE): the Hall conductivity of thin samples at very low temperatures, exposed to strong transverse magnetic fields, is equal to an integer times the von Klitzing constant $e^{2} / h,[43]$. This measurement is amazingly sharp: the observation of the Hall plateaux is by now used to measure the fine structure constant, at a very high level of accuracy. In view of the complexity of the underlying microscopic Hamiltonian, which depends on several material- and sample-dependent parameters, the universality of the Hall conductivity is a very remarkable phenomenon.

The quantization of the Hall conductivity for non-interacting fermions has a deep topological interpretation $[4,53]$, and the intrinsic robustness of a topological quantity offers a natural qualitative explanation of the observed universality. The universality of the Hall conductivity in the presence of disorder has later been established in full mathematical rigor in $[2,6,8]$.

A similar universality property is expected to be valid in the presence of many-body interactions, as well. However, while in the non-interacting case the features of the many-body problem can be deduced from the single-particle Schrödinger operator, in the interacting case one needs to consider the full $N$-particle Schrödinger equation, which is much harder to 
study. This explains why a mathematical proof of the quantization of the Hall conductance for interacting electrons remained open [5] for many years.

Effective field theories $[15,26-29,55,56]$, have been used for explaining a possible topological mechanisms underlying both the integral and the fractional QHE in interacting electron systems. However, they are based on certain phenomenological assumptions, such as the incompressibility of the "quantum Hall fluid", which may be very hard to check from first principles in concrete models.

More recently, the quantization of the Hall conductivity has been rigorously proved [38]. The proof of [38] is based on the hypothesis that the interacting ground state is nondegenerate and, as in the effective field theory approach, incompressible, which amounts to say that the interacting ground state is gapped, uniformly in the system size. This assumption is unproven in most physically relevant cases, at least in the context of interacting fermions. As far as we know, the only cases for which it is proved are perturbations of "topologically trivial" classical reference states [24, 25], or of "frustration free" Hamiltonians [16-18, 48], that is of Hamiltonians that can be written as sums of projectors geometrically localized around the sites of the underlying lattice.

In this work, by using a different approach, we prove the quantization of the Hall conductivity for general interacting fermionic systems, under the assumptions that the reference non-interacting system is gapped, and that the interaction is weak and short-ranged. In particular, our result applies to the interacting versions of the Hofstadter [1, 39] and Haldane [37] models. See also [54] and [41] for numerical and experimental results on the interacting Haldane model. We stress that our proof does not require any a priori assumption on the interacting spectrum of the system. It is based on constructive cluster expansion techniques combined with lattice Ward Identities. We write a convergent power series expansion for the conductivity, defined in terms of the Kubo formula, and we show that all the interactiondependent corrections vanish exactly, in the infinite volume and zero temperature limits.

The idea that the universality of Hall conductance follows from Ward Identities is well known [23, 40]. However, their implementation was so far limited to continuum effective quantum field theory models plagued by ultraviolet divergences, and their use was combined with formal manipulations of non-convergent Feynman graph expansions. Here, we consider lattice Hamiltonian models, and we develop a strategy similar to [23, 40], based on lattice Ward Identities. The convergence of the perturbative series is achieved by re-summing the usual Feynman diagram expansions in the form of a suitable determinant expansion, which admits improved combinatorial estimates. Similar techniques, combined with an infrared Renormalization Group analysis, were used earlier for constructing the ground state of several low-dimensional interacting Fermi systems, and for proving universality relations among critical exponents, amplitudes and conductivities [9-14, 35, 36, 47]. In this paper we apply these ideas for the first time to the study of the transverse (Hall) conductivity.

An informal statement of our main result is the following. 
Consider a fermionic system on a two-dimensional periodic lattice, with grand canonical Hamiltonian $H_{0}+U V$, where $H_{0}$ is a quadratic gapped Hamiltonian, $V$ is a density-density interaction, decaying faster than any power at large distances, and $U$ is its strength. If $U$ is small enough, then the interacting correlation functions are analytic in $U$ and decay faster than any power at large distances, uniformly in the system size and in the temperature. The conductivity matrix, defined by the Kubo formula, is analytic as well, and its infinite volume and zero temperature limit is independent of $U$. In particular, the longitudinal conductivity is zero, while the transverse one is quantized.

The rest of the paper is devoted to the proof of this result. In section 2 we define the general class of Hamiltonians we consider, we define the current observable and the conductivity, and state our main theorem in a mathematically precise way. In section 3, we introduce the imaginary-time counterpart of the conductivity and state a "reconstruction theorem" that guarantees its equivalence with the standard (real-time) Kubo conductivity. In section 4 , we prove the quantization of the conductivity, under the assumption of analyticity and smoothness of the multipoint current correlations at imaginary times. The key ingredient in the proof is the use of Ward Identites, which are nothing but the restatement of the continuity equation for the density at the level of correlation functions. In section 5 we prove the analyticity and smoothness of the imaginary-time/Matsubara frequency correlations, by using multiscale fermionic cluster expansion techniques. Strictly speaking, the content of section 5 is a straightforward adaptation of previous results, but we include it here for the sake of self-containedness. In section 6 we prove the reconstruction theorem stated in section 3, thus concluding the proof of our main result. In the appendices we collect some auxiliary results: in appendix A we reproduce the well known result that the non-interacting Kubo conductivity is equal to the Chern number of the filled Bloch bands; in appendix B we apply our main result to the interacting Haldane model, and show that it displays a non-trivial topological phase diagram; in appendix $\mathrm{C}$ we discuss the existence of the infinite volume dynamics, required in the proof of the reconstruction theorem.

\section{THE MODEL AND THE MAIN RESULT}

In this section, we give a mathematically precise formulation of the class of models we consider. First, we introduce the periodic lattice and the fermionic operators associated with its sites. Next, we define the grand-canonical Hamiltonian and state our main assumptions on its quadratic and interaction parts, including the gap condition for the non-interacting theory. We proceed by introducing the current and conductivity observables, and finally we state our main result. 


\section{A. Lattice fermionic operators}

We let $\Lambda=\left\{n_{1} \vec{\ell}_{1}+n_{2} \vec{\ell}_{2}, n_{i} \in \mathbb{Z}\right\}$ be the Bravais lattice generated by the two linearly independent vectors $\vec{\ell}_{1}, \vec{\ell}_{2} \in \mathbb{R}^{2}$. Given $L \in \mathbb{N}$, we also let $\Lambda_{L}=\Lambda / L \Lambda$ be the corresponding finite torus of side $L$, which can be thought of as the set

$$
\Lambda_{L}=\left\{\vec{x} \mid \vec{x}=n_{1} \vec{\ell}_{1}+n_{2} \vec{\ell}_{2}, n_{i} \in \mathbb{Z}, 0 \leq n_{i}<L\right\}
$$

with periodic boundary conditions (i.e., endowed with the euclidean distance on the torus, denoted by $\left.|\vec{x}-\vec{y}|_{L}=\min _{\vec{n} \in \mathbb{Z}^{2}}\left|\vec{x}-\vec{y}+n_{1} \vec{\ell}_{1} L+n_{2} \overrightarrow{\ell_{2}} L\right|\right)$. The number of sites of $\Lambda_{L}$ is denoted by $\left|\Lambda_{L}\right|=L^{2}$. With each site $\vec{x} \in \Lambda_{L}$, we associate fermionic creation and annihilation operators $\psi_{\vec{x}, \sigma}^{ \pm}$, with $\sigma \in I$, and $I$ a finite set of indices, which can be thought of as "color" labels, possibly corresponding to the spin, or to different sublattices. In particular, the fermion labeled by $\sigma$ can be thought of as living on a physical lattice obtained by translating $\Lambda_{L}$ by a fixed amount $\vec{r}_{\sigma} \in \mathbb{R}$ (possibly equal to $\overrightarrow{0}$, in the case that, e.g., $\sigma$ is a spin index).

The fermionic operators satisfy the usual canonical anticommutation relations:

$$
\left\{\psi_{\vec{x}, \sigma}^{\varepsilon}, \psi_{\vec{y}, \sigma^{\prime}}^{\varepsilon^{\prime}}\right\}=\delta_{\varepsilon,-\varepsilon^{\prime}} \delta_{\vec{x}, \vec{y}} \delta_{\sigma, \sigma^{\prime}}
$$

where $\varepsilon, \varepsilon^{\prime}= \pm, \vec{x}, \vec{y} \in \Lambda_{L}, \sigma, \sigma^{\prime} \in I$, and $\delta$., is the Kronecker delta. Consistently with the periodic boundary conditions, we identify the fermionic operators obtained by translating $\vec{x}$ by an integer multiple of $L \vec{\ell}_{i}$. We let $\vec{G}_{1}, \vec{G}_{2}$ be a basis of the reciprocal lattice $\Lambda_{L}^{*}$ of $\Lambda$, i.e., $\vec{G}_{i} \cdot \vec{\ell}_{j}=2 \pi \delta_{i, j}$, and we define the finite-volume Brillouin zone as

$$
\mathcal{B}_{L}:=\left\{\vec{k} \mid \vec{k}=\frac{n_{1}}{L} \vec{G}_{1}+\frac{n_{2}}{L} \vec{G}_{2}, n_{i} \in \mathbb{Z}, 0 \leq n_{i}<L\right\} .
$$

We will also denote $\mathcal{B}=\mathcal{B}_{\infty}$. We let the Fourier transforms of the fermionic operators be:

$$
\psi_{\vec{x}, \sigma}^{ \pm}=\frac{1}{L^{2}} \sum_{\vec{k} \in \mathcal{B}_{L}} e^{ \pm i \vec{k} \cdot \vec{x}} \hat{\psi}_{\vec{k}, \sigma}^{ \pm}, \quad \forall \vec{x} \in \Lambda_{L}, \quad \Longleftrightarrow \quad \hat{\psi}_{\vec{k}, \sigma}^{ \pm}=\sum_{\vec{x} \in \Lambda_{L}} e^{\mp i \vec{k} \cdot x} \psi_{\vec{x}, \sigma}^{ \pm}, \quad \forall \vec{k} \in \mathcal{B}_{L}
$$

Note that, with this definition, the fermionic operators in momentum space are periodic over the first Brillouin zone, that is $\hat{\psi}_{\vec{k}, \sigma}^{ \pm}=\hat{\psi}_{\vec{k}+\vec{G}_{i}, \sigma}^{ \pm}, i=1,2$. Moreover,

$$
\left\{\hat{\psi}_{\vec{k}, \sigma}^{\varepsilon}, \hat{\psi}_{\vec{k}^{\prime}, \sigma^{\prime}}^{\varepsilon^{\prime}}\right\}=L^{2} \delta_{\varepsilon,-\varepsilon^{\prime}} \delta_{\vec{k}, \vec{k}^{\prime}} \delta_{\sigma, \sigma^{\prime}}
$$

\section{B. The Hamiltonian and the Gibbs state}

The grand-canonical Hamiltonian of the system is assumed to be of the form:

$$
\mathcal{H}_{L}-\mu \mathcal{N}_{L}=\mathcal{H}_{L}^{(0)}+U \mathcal{V}_{L}-\mu \mathcal{N}_{L}
$$


with

$$
\begin{aligned}
& \mathcal{H}_{L}^{(0)}=\sum_{\vec{x}, \vec{y} \in \Lambda_{L}} \sum_{\sigma, \sigma^{\prime} \in I} \psi_{\vec{x}, \sigma}^{+} H_{\sigma \sigma^{\prime}}^{(0)}(\vec{x}-\vec{y}) \psi_{\vec{y}, \sigma^{\prime}}^{-}, \\
& \mathcal{V}_{L}=\sum_{\vec{x}, \vec{y} \in \Lambda_{L}} \sum_{\sigma, \sigma^{\prime} \in I} n_{\vec{x}}^{\sigma} v_{\sigma \sigma^{\prime}}(\vec{x}-\vec{y}) n_{\vec{y}}^{\sigma^{\prime}}, \quad \text { where } n_{\vec{x}}^{\sigma}=\psi_{\vec{x}, \sigma}^{+} \psi_{\vec{x}, \sigma}^{-}, \\
& \text {and } \quad \mathcal{N}_{L}=\sum_{\vec{x} \in \Lambda_{L}} \sum_{\sigma \in I} n_{\vec{x}}^{\sigma} .
\end{aligned}
$$

The operator $\mathcal{H}_{L}^{(0)}$ is called the free Hamiltonian, while $U \mathcal{V}_{L}$ is the many-body interaction, and $U$ plays the role of the interaction strength. The constant $\mu$ is the chemical potential, or Fermi level.

We assume the hopping function $H_{\sigma \sigma^{\prime}}^{(0)}(\vec{x})=H_{L ; \sigma \sigma^{\prime}}^{(0)}(\vec{x})$ to be a function on the torus (i.e., a periodic function on $\left.\Lambda_{L}\right)$, such that $H_{\sigma \sigma^{\prime}}^{(0)}(\vec{x})=\sum_{\vec{n} \in \mathbb{Z}^{2}} H_{\infty ; \sigma \sigma^{\prime}}^{(0)}\left(\vec{x}+n_{1} \vec{\ell}_{1} L+n_{2} \vec{\ell}_{2} L\right)$, and $H_{\sigma \sigma}^{(0)}(\overrightarrow{0})=0$. In order for the free Hamiltonian to be self-adjoint, we require $\left[H_{\sigma \sigma^{\prime}}^{(0)}(\vec{x})\right]^{*}=$ $H_{\sigma^{\prime} \sigma}^{(0)}(-\vec{x})$. Moreover, we assume that $H_{\infty ; \sigma \sigma^{\prime}}^{(0)}$ decays faster than any power at large distances, so that:

$$
\left\|H^{(0)}(\vec{x})\right\| \leq \frac{C_{N}}{1+|\vec{x}|_{L}^{N}}, \quad \forall N \geq 0 .
$$

As a consequence of these assumptions, we see that the Bloch Hamiltonian

$$
\hat{H}^{(0)}(\vec{k}):=\sum_{\vec{x} \in \Lambda_{L}} e^{i \vec{k} \cdot \vec{x}} H^{(0)}(\vec{x})
$$

is a self-adjoint matrix, so that the spectrum $\sigma\left(\hat{H}^{(0)}(\vec{k})\right)=\left\{\varepsilon_{\sigma}(\vec{k})\right\}_{\sigma \in I}$ is real. The functions $\vec{k} \mapsto \varepsilon_{\sigma}(\vec{k})$ are called the energy bands. We let

$$
\mathfrak{e}_{0}=\sup _{\vec{k} \in \mathcal{B}}\left\|\hat{H}^{(0)}(\vec{k})\right\|
$$

which sets the energy scale. Note also that the infinite volume limit of $\hat{H}^{(0)}(\vec{k})$ is infinitely differentiable in $\vec{k}$.

Concerning the interaction, we assume, similarly, that $v_{\sigma \sigma^{\prime}}(\vec{x})$ is periodic on $\Lambda_{L}$, equal to the sum over the images of its infinite volume limit, such that $v_{\sigma \sigma}(\overrightarrow{0})=0, v_{\sigma \sigma^{\prime}}(\vec{x}-\vec{y})=$ $v_{\sigma^{\prime} \sigma}(\vec{y}-\vec{x})$ and

$$
\|v(\vec{x})\| \leq \frac{C_{N}}{1+|\vec{x}|_{L}^{N}}, \quad \forall N \geq 0 .
$$

In particular, the infinite volume limit of

$$
\hat{v}_{\sigma \sigma^{\prime}}(\vec{p})=\sum_{\vec{x} \in \Lambda_{L}} e^{i \vec{p} \cdot \vec{x}} v_{\sigma \sigma^{\prime}}(\vec{x})
$$

is infinitely differentiable in $\vec{p}$. 
Finally, concerning the choice of the Fermi level, we assume the following gap condition:

$$
\delta_{\mu}=\inf _{\vec{k} \in \mathcal{B}} \operatorname{dist}\left(\mu, \sigma\left(\hat{H}^{(0)}(\vec{k})\right)\right)>0 .
$$

One of the important implications of the gap condition is that the projector over the "ffilled bands", i.e., over the bands with energy smaller than $\mu$, is smooth in $\vec{k}$ : more precisely, the operator $P_{-}(\vec{k})=\sum_{\alpha: \varepsilon_{\alpha}(\vec{k})<\mu} P_{\alpha}(\vec{k})$, with $P_{\alpha}(\vec{k})$ the projector over the $\alpha$-th energy band, is a projector itself and, in the infinite volume limit, it is infinitely differentiable in $\vec{k}$.

Remark. The assumptions that $H^{(0)}(\vec{x})$ and $v(\vec{x})$ decay faster than any power are far from being optimal. It is easy to adapt the following discussion to the case of sufficiently fast power-law decay of the hopping matrix and of the interaction. Since we are not interested in optimal bounds, for simplicity we illustrate our method in the case of faster-than-any-power decay. Note also that, if the hopping matrix decays exponentially fast at large distances, then the Bloch Hamiltonian and the projector $P_{-}(\vec{k})$ are analytic in $\vec{k}$, rather than just infinitely differentiable.

In the following, we construct the grand-canonical Gibbs state associated with (2.6), which is characterized by its correlation functions, defined as follows. Given an observable O, that is a self-adjoint operator on the fermionic Fock space $\mathcal{F}$, its expectation value is:

$$
\langle O\rangle_{\beta, \mu, L}:=\operatorname{Tr}_{\mathcal{F}} \rho_{\beta, \mu, L} O, \quad \rho_{\beta, \mu, L}=\frac{e^{-\beta\left(\mathcal{H}_{L}-\mu \mathcal{N}_{L}\right)}}{\operatorname{Tr}_{\mathcal{F}} e^{-\beta\left(\mathcal{H}_{L}-\mu \mathcal{N}_{L}\right)}},
$$

where $\mathcal{F}$ is the fermionic Fock space. The chemical potential $\mu$ should be thought of as being fixed once and for all, so that (2.13) is verified. Therefore, for notational convenience, we shall drop the label $\mu$ from the symbol for the Gibbs state and for the density matrix: $\langle\cdot\rangle_{\beta, \mu, L} \equiv\langle\cdot\rangle_{\beta, L}$ and $\rho_{\beta, \mu, L} \equiv \rho_{\beta, L}$.

\section{The current and the conductivity}

Let us preliminarily define the current observable on the infinite lattice $\Lambda$. Given $\vec{x} \in \Lambda$, we let $\vec{x}_{\sigma}:=\vec{x}+\vec{r}_{\sigma}$ be the location of the fermion labeled by $\sigma \in I$, see the discussion following (2.1). The total position operator is defined as $\vec{X}=\sum_{\sigma \in I} \sum_{\vec{x} \in \Lambda} \vec{x}_{\sigma} n_{\vec{x}}^{\sigma}$, while the d.c. current is

$$
\vec{J}:=i[\mathcal{H}, \vec{X}]
$$

where $\mathcal{H}$ is the formal infinite volume limit of $\mathcal{H}_{L}$. Note that $\vec{J}=i\left[\mathcal{H}^{(0)}, \vec{X}\right]$, because $\mathcal{V}$ commutes with $\vec{X}$. Moreover, $\vec{X}$ can be naturally decomposed as $\vec{X}=\vec{X}^{(1)}+\vec{X}^{(2)}$, where $\vec{X}^{(1)}=\sum_{\vec{x}, \sigma} \vec{x} n_{\vec{x}}^{\sigma}$ represents the position of the "centers" of the cells of the Bravais lattice, and $\vec{X}^{(2)}$ the displacement with respect to the centers. The current $\vec{J}$ inherits this decomposition. As we shall see below, the "displacement current" $\vec{J}^{(2)}$ does not contribute to the conductivity, in the infinite volume and zero temperature limits. 
By using the definition of $\mathcal{H}$, we can rewrite $\vec{J}$ more explicitly as

$$
\vec{J}=\frac{1}{2} \sum_{\vec{x}, \vec{y} \in \Lambda} \sum_{\sigma, \sigma^{\prime} \in I}\left(\vec{y}_{\sigma^{\prime}}-\vec{x}_{\sigma}\right) J_{\vec{x} \vec{y}}^{\sigma \sigma^{\prime}}
$$

where

$$
J_{\vec{x} \vec{y}}^{\sigma \sigma^{\prime}}=i\left[\psi_{\vec{x}, \sigma}^{+} H_{\sigma \sigma^{\prime}}^{(0)}(\vec{x}-\vec{y}) \psi_{\vec{y}, \sigma^{\prime}}^{-}-\psi_{\vec{y}, \sigma^{\prime}}^{+} H_{\sigma^{\prime} \sigma}^{(0)}(\vec{y}-\vec{x}) \psi_{\vec{x}, \sigma}^{-}\right]
$$

is the bond current flowing from $\vec{x}_{\sigma}$ to $\vec{y}_{\sigma^{\prime}}$. The bond current satisfies a natural continuity equation, which is reviewed in the next section. The finite volume current (to be still denoted by $\vec{J}$ in the following, with some abuse of notation) is defined by an expression analogous to (2.16), with the sums over $\vec{x}$ and $\vec{y}$ restricted to $\Lambda_{L}$, and the vector $\left(\vec{y}_{\sigma^{\prime}}-\vec{x}_{\sigma}\right)$ to be interpreted as $(\vec{y}-\vec{x})_{L}+\vec{r}_{\sigma^{\prime}}-\vec{r}_{\sigma}$, where, if $\vec{y}-\vec{x}=\left(n_{1} \vec{\ell}_{1}, n_{2} \vec{\ell}_{2}\right)$, then $(\vec{y}-\vec{x})_{L}=\left(\left\{n_{1}\right\}_{L} \vec{\ell}_{1},\left\{n_{2}\right\}_{L} \vec{\ell}_{2}\right)$, with $\{n\}_{L}=n-L\left\lfloor\frac{n}{L}+\frac{1}{2}\right\rfloor$.

The conductivity matrix in the infinite volume and zero temperature limits is defined via the Kubo formula [44] as:

$$
\sigma_{i j}(U)=\frac{1}{A} \lim _{\omega \rightarrow 0^{+}} \frac{1}{\omega}\left(i \int_{-\infty}^{0} d t e^{\omega t}\left\langle\left[e^{i \mathcal{H} t} J_{i} e^{-i \mathcal{H} t}, J_{j}\right]\right\rangle_{\infty}-\left\langle\left[\left[\mathcal{H}, X_{i}\right], X_{j}\right]\right\rangle_{\infty}\right),
$$

where $A=\left|\vec{\ell}_{1} \wedge \vec{\ell}_{2}\right|$ is the area of the fundamental cell, and $\langle O\rangle_{\infty}=\lim _{\beta \rightarrow \infty} \lim _{L \rightarrow \infty} L^{-2}\left\langle O_{L}\right\rangle_{\beta, L}$. The second term in parentheses is known as the diamagnetic term, or Schwinger term. This formula describes the response of the system at $t=0$ after adiabatically switching on at $t=-\infty$ a time-dependent external field, whose amplitude is damped by a factor $e^{\omega t}$, see $[2$, Eq.(A.7)].

\section{The main result}

We are finally in the position of stating our main result in a mathematically precise way.

Theorem 2.1 [Universality of the conductivity matrix] Let $\sigma_{i j}(U)$ be the conductivity matrix of the model with Hamiltonian (2.6), as defined in (2.18). Under the assumptions on the Hamiltonian spelled out after (2.7) (see (2.8), (2.11) and (2.13)), there exists $U_{0}>0$ such that

$$
\sigma_{i j}(U)=\sigma_{i j}(0), \quad \forall i, j=1,2,
$$

as long as $U \in\left(-U_{0}, U_{0}\right)$.

\section{Remarks.}

- The non-interacting conductivity $\sigma_{i j}(0)$ is well-known to be quantized $[2,4,53]$. The proof that the non-interacting conductivity is equal to the first Chern number is reproduced for completeness in appendix A. Therefore, theorem 2.1 tells us that, if 
$|U| \leq U_{0}, \sigma_{i j}(U) \in \mathbb{Z} /(2 \pi)$, which is the usual quantization formula of the Hall conductivity, in units where $e=\hbar=1$. Moreover, the computation in appendix A shows that $\vec{J}^{(2)}$, which was defined after $(2.15)$, gives a vanishing contribution to $\sigma_{i j}(0)$ and, therefore, in light of theorem 2.1, it gives vanishing contribution to the interacting conductivity, as well.

- The infinite volume and zero temperature current correlations entering the definition of conductivity are defined by first sending the volume to infinity and then the temperature to zero. However, in the situation we are considering, the two limits can be interchanged, thanks to the gap condition.

\section{IMAGINARY TIMES AND MATSUBARA FREQUENCIES}

In this section we introduce the notion of Euclidean (imaginary-time) correlation functions, which are the most natural class of correlations that can be studied by the many-body thermodynamic formalism. We also introduce their Fourier transform, which are known as the correlations at imaginary (or "Matsubara") frequencies. Next, we define the imaginary time/Matsubara frequency counterpart of the Kubo conductivity, and state a "reconstruction theorem" about the equivalence between the two definitions of conductivity, to be proved in section 6 .

\section{A. Euclidean correlation functions}

Given an observable $O$, we let $O_{t}$ with $t \in[0, \beta)$ be its imaginary time evolution, namely

$$
O_{t}:=e^{t\left(\mathcal{H}_{L}-\mu \mathcal{N}_{L}\right)} O e^{-t\left(\mathcal{H}_{L}-\mu \mathcal{N}_{L}\right)} .
$$

Given $n$ observables $O_{t_{1}}^{(1)}, \ldots, O_{t_{n}}^{(n)}$, each of which can be written as a polynomial in the time-evolved creation and annihilation operators $\psi_{(t, \vec{x}), \sigma}^{ \pm}=e^{t\left(\mathcal{H}_{L}-\mu \mathcal{N}_{L}\right)} \psi_{\vec{x}, \sigma}^{ \pm} e^{-t\left(\mathcal{H}_{L}-\mu \mathcal{N}_{L}\right)}$, we define their time-ordered average as:

$$
\left\langle\mathbf{T} O_{t_{1}}^{(1)} \cdots O_{t_{n}}^{(n)}\right\rangle_{\beta, L}:=\frac{\operatorname{Tr}_{\mathcal{F}} e^{-\beta\left(\mathcal{H}_{L}-\mu \mathcal{N}_{L}\right)} \mathbf{T}\left\{O_{t_{1}}^{(1)} \cdots O_{t_{n}}^{(n)}\right\}}{\operatorname{Tr}_{\mathcal{F}} e^{-\beta\left(\mathcal{H}_{L}-\mu \mathcal{N}_{L}\right)}},
$$

where the (linear) operator $\mathbf{T}$ is the fermionic time-ordering, acting on a product of fermionic operators as:

$$
\mathbf{T}\left\{\psi_{\left(t_{1}, \vec{x}_{1}\right), \sigma_{1}}^{\varepsilon_{1}} \cdots \psi_{\left(t_{n}, \vec{x}_{n}\right), \sigma_{n}}^{\varepsilon_{n}}\right\}=\operatorname{sgn}(\pi) \psi_{\left(t_{\pi(1)}, \vec{x}_{\pi(1)}\right), \sigma_{\pi(1)}}^{\varepsilon_{\pi(1)}} \cdots \psi_{\left(t_{\pi(n)}, \vec{x}_{\pi(n)}\right), \sigma_{\pi(n)}}^{\varepsilon_{\pi(n)}},
$$

where $\pi$ is a permutation of $\{1, \ldots, n\}$ with signature $\operatorname{sgn}(\pi)$ such that $t_{\pi(1)} \geq \ldots \geq t_{\pi(n)}$. If some operators are evaluated at the same time, the ambiguity is solved by normal ordering.

Moreover, we denote by $\left\langle\mathbf{T} O_{t_{1}}^{(1)} ; \cdots ; O_{t_{n}}^{(n)}\right\rangle_{\beta, L}$ the time-ordered truncated correlation function, or cumulant, of $O_{t_{1}}^{(1)}, \ldots, O_{t_{n}}^{(n)}$. If the observables are all even, i.e., if they are linear 
combinations of even monomials in the creation and annihiliation operators, the cumulant is defined as follows [3]:

$$
\left\langle\mathbf{T} O_{t_{1}}^{(1)} ; O_{t_{2}}^{(2)} ; \cdots ; O_{t_{n}}^{(n)}\right\rangle:=\left.\frac{\partial^{n}}{\partial \lambda_{1} \cdots \partial \lambda_{n}} \log \left\{1+\sum_{I \subseteq\{1,2, \ldots, n\}} \lambda(I)\langle\mathbf{T} O(I)\rangle\right\}\right|_{\boldsymbol{\lambda}=\mathbf{0}},
$$

where $\lambda(I)=\prod_{i \in I} \lambda_{i}$ and $O(I)=\prod_{i \in I} O_{t_{i}}^{(i)}$. For $n=1$, this definition reduces to $\left\langle O_{t_{1}}^{(1)}\right\rangle=$ $\left\langle O^{(1)}\right\rangle$. For $n=2$ one gets $\left\langle\mathbf{T} O_{t_{1}}^{(1)} ; O_{t_{2}}^{(2)}\right\rangle=\left\langle\mathbf{T} O_{t_{1}}^{(1)} O_{t_{2}}^{(2)}\right\rangle-\left\langle O_{t_{1}}^{(1)}\right\rangle\left\langle O_{t_{2}}^{(2)}\right\rangle$, and so on. A similar definition of time-ordered truncated expectation is valid in the case that $O_{t_{i}}^{(i)}$ is replaced by an operator depending on multiple times, e.g., by $O_{t_{i}}^{(i)} \tilde{O}_{t_{i}^{\prime}}^{(i)}$, with $\tilde{O}^{(i)}$ another even observable.

We also introduce the notion of Fourier transform with respect to the imaginary time. Consider, again, the case of $n$ even observables $O_{t_{1}}^{(1)}, \ldots, O_{t_{n}}^{(n)}$, with $t_{i} \in[0, \beta)$. We denote by $\widehat{O}_{\omega_{i}}^{(i)}:=\int_{0}^{\beta} d t e^{-i \omega_{i} t} O_{t}^{(i)}$ their Fourier transforms, where $\omega_{i} \in \frac{2 \pi}{\beta} \mathbb{Z}$ are called Matsubara frequencies. By using the definition of time-ordered correlations and the cyclicity of the trace, it is straightforward to check that

$$
\begin{aligned}
\int_{0}^{\beta} d t_{1} \cdots & \int_{0}^{\beta} d t_{n} e^{-i \omega_{1} t_{1} \cdots-i \omega_{n} t_{n}}\left\langle\mathbf{T} O_{t_{1}}^{(1)} ; \cdots ; O_{t_{n}}^{(n)}\right\rangle_{\beta, L}= \\
& =\delta_{\omega_{1}+\cdots+\omega_{n}, 0}\left\langle\mathbf{T} \widehat{O}_{\omega_{1}}^{(1)} ; \cdots ; \widehat{O}_{\omega_{n-1}}^{(n-1)} ; \widehat{O}_{-\left(\omega_{1}+\cdots+\omega_{n-1}\right)}^{(n)}\right\rangle_{\beta, L},
\end{aligned}
$$

which sets our convention on the Fourier transform of the truncated correlations.

\section{B. The continuity equation}

As anticipated in section $2 \mathrm{C}$, the (imaginary-time) evolution of the bond current satisfies a natural continuity equation, which reads as follows: if $n_{(t, \vec{x})}^{\sigma}$ and $\left(J_{\vec{x}}^{\sigma \sigma^{\prime}}\right)_{t}$ are the imaginary time evolutions of $n_{\vec{x}}^{\sigma}$ and of $J_{\vec{x}}^{\sigma \sigma^{\prime}}$, respectively, then

$$
\partial_{t} n_{(t, \vec{x})}^{\sigma}=i \sum_{\vec{y} \in \Lambda_{L}} \sum_{\sigma^{\prime} \in I}\left(J_{\vec{x} \vec{y}}^{\sigma \sigma^{\prime}}\right)_{t} .
$$

The continuity equations (3.6) for different values of $\sigma$ can be conveniently combined in a single equation, by letting

$$
\tilde{J}_{0,(t, \vec{p})}:=\sum_{\vec{x} \in \Lambda_{L}} \sum_{\sigma \in I} e^{-i \vec{p} \cdot \vec{x}_{\sigma}} n_{(t, \vec{x})}^{\sigma},
$$

with $\vec{p} \in\left\{\vec{k}: \vec{k}=\frac{n_{1}}{L} \vec{G}_{1}+\frac{n_{2}}{L} \vec{G}_{2}, n_{i} \in \mathbb{Z}\right\}$. The observable $\tilde{J}_{0,(t, \vec{p})}$ satisfies

$$
\partial_{t} \tilde{J}_{0,(t, \vec{p})}+\vec{p} \cdot \overrightarrow{\tilde{J}}_{(t, \vec{p})}=0
$$

where

$$
\overrightarrow{\tilde{J}}_{(t, \vec{p})}=\frac{1}{2} \sum_{\vec{x}, \vec{y} \in \Lambda_{L}} \sum_{\sigma, \sigma^{\prime} \in I} e^{-i \vec{p} \cdot \vec{x}_{\sigma}}\left(\vec{y}_{\sigma^{\prime}}-\vec{x}_{\sigma}\right) \eta_{\vec{x} \vec{y}}^{\sigma \sigma^{\prime}}(\vec{p})\left(J_{\vec{x}}^{\sigma \sigma^{\prime}}\right)_{t}
$$


and

$$
\eta_{\vec{x} \vec{y}}^{\sigma \sigma^{\prime}}(\vec{p})=\frac{1-e^{-i \vec{p} \cdot\left(\vec{y}_{\sigma^{\prime}}-\vec{x}_{\sigma}\right)}}{i \vec{p} \cdot\left(\vec{y}_{\sigma^{\prime}}-\vec{x}_{\sigma}\right)},
$$

with the understanding that $\eta_{\vec{x} \vec{y}}^{\sigma \sigma^{\prime}}(\overrightarrow{0})=1$, if $\vec{y}_{\sigma^{\prime}} \neq \vec{x}_{\sigma}$, and $\eta_{\vec{x} \vec{y}}^{\sigma \sigma^{\prime}}(\vec{p})=0$, if $\vec{y}_{\sigma^{\prime}}=\vec{x}_{\sigma}$. Note that, in general, $\tilde{J}_{0,(t, \vec{p})}$ and $\overrightarrow{\tilde{J}}_{(t, \vec{p})}$ are not periodic in $\vec{p}$ over the Brillouin zone.

\section{The conductivity at imaginary frequency. A reconstruction theorem}

The natural counterpart of the Kubo conductivity (2.18) at imaginary time/Matsubara frequency is defined as follows (cf., e.g., [46, Eqs.(3.388) to (3.391)] and [52, Eqs.(6) to (10)]):

$$
\bar{\sigma}_{i j}(U):=-\lim _{\omega \rightarrow 0^{+}} \frac{1}{A} \frac{1}{\omega}\left[\widehat{K}_{i j}(\omega, \overrightarrow{0})-\widehat{K}_{i j}(0, \overrightarrow{0})\right]
$$

where

$$
\widehat{K}_{i j}(\omega, \vec{p})=\lim _{\beta \rightarrow \infty} \lim _{L \rightarrow \infty} \frac{1}{\beta L^{2}}\left\langle\mathbf{T} \hat{J}_{i,(\omega, \vec{p})} ; \hat{J}_{j,(-\omega,-\vec{p})}\right\rangle_{\beta, L}
$$

and $\hat{J}_{i,(\omega, \vec{p})}=\int_{0}^{\beta} d t e^{-i \omega t} \tilde{J}_{i,(t, \vec{p})}$ is the Fourier transform of the current-current correlation. Note that the labels $i, j \in\{1,2\}$ in the previous two equations refer to the basis $\hat{e}_{1}=(1,0)$, $\hat{e}_{2}=(0,1)$. Now, if the infinite-volume current-current correlation function $\widehat{K}_{i j}(\omega, \overrightarrow{0})$ is differentiable at $\omega=0$, then $(3.11)$ reduces to:

$$
\bar{\sigma}_{i j}(U)=-\frac{1}{A} \frac{\partial \widehat{K}_{i j}}{\partial \omega}(0, \overrightarrow{0}), \quad i=1,2 .
$$

This is in fact the case for the class of systems we are considering, as we shall prove on the basis of fermionic cluster expansion methods, by taking advantage of the gap condition (2.13) on the non-interacting spectrum. See proposition 4.1 below.

Remarkably, for the class of gapped systems we are considering, the Kubo conductivity at imaginary frequency, in the limit of zero frequency, is the same as its real-time counterpart $\sigma_{i j}(U)$ defined in (2.18). This is summarized in the following proposition.

Theorem 3.1 [Reconstruction of the real-time Kubo formula] Under the same assumptions as theorem 2.1,

$$
\bar{\sigma}_{i j}(U)=\sigma_{i j}(U)
$$

for all $U \in\left(-U_{0}, U_{0}\right)$.

This theorem will proved in section 6. It allows us to study the Kubo conductivity via its imaginary time counterpart, which is more directly accessible to constructive many-body techniques, to be described in the following two sections. 


\section{UNIVERSALITY AND WARD IDENTITIES}

In this section, we prove theorem 2.1, by combining the use of exact lattice Ward Identities with the information that the multipoint density and current correlations are analytic in $U$ and smooth in the momenta. More in detail, we first introduce the definition of multipoint density and current correlations, and state a result, to be proved in section 5 , concerning the regularity of these correlations. Next, we introduce the notion of Ward Identities, and prove an important consequence thereof, in the form of an identity relating certain correlations to the derivatives of other correlation functions. We then proceed to prove the so-called Schwinger-Dyson equation for the correlation functions. Finally, we put together all these ingredients and prove that $\bar{\sigma}_{i j}(U)=\bar{\sigma}_{i j}(0)$, for $U$ small enough. In light of theorem 3.1, this implies theorem 2.1 for $\sigma_{i j}(U)$.

\section{A. Multipoint density and current correlation functions}

For $n \geq 2$, we let $\mathbf{p}_{i}=\left(\omega_{i}, \vec{p}_{i}\right) \in(2 \pi / \beta) \mathbb{Z} \times \mathcal{D}_{L}$ and $\alpha_{i} \in\{0,1,2\} \cup I$, with $\mathcal{D}_{L}=\{\vec{k}:$ $\left.\vec{k}=\frac{n_{1}}{L} \vec{G}_{1}+\frac{n_{2}}{L} \vec{G}_{2}, n_{i} \in \mathbb{Z}\right\}$ and $i=1, \ldots, n-1$, and we define:

$$
\widehat{K}_{\alpha_{1}, \ldots, \alpha_{n}}^{\beta, L}\left(\mathbf{p}_{1}, \ldots, \mathbf{p}_{n-1}\right):=\frac{1}{\beta L^{2}}\left\langle\mathbf{T} \hat{J}_{\alpha_{1}, \mathbf{p}_{1}} ; \cdots ; \hat{J}_{\alpha_{n-1}, \mathbf{p}_{n-1}} ; \hat{J}_{\alpha_{n},-\mathbf{p}_{1}-\ldots-\mathbf{p}_{n-1}}\right\rangle_{\beta, L}
$$

where, if $\alpha=\mu \in\{0,1,2\}$, then $\hat{J}_{\mu, \mathbf{p}}=\int_{0}^{\beta} d t e^{-i \omega t} \tilde{J}_{\mu,(t, \vec{p})}$, with $\tilde{J}_{\mu,(t, \vec{p})}$ as in (3.7) and (3.9), while, if $\alpha=\sigma \in I$, then $\hat{J}_{\sigma, \mathbf{p}}=\hat{n}_{\mathbf{p}}^{\sigma}=\int_{0}^{\beta} d t e^{-i \omega t} \tilde{n}_{(t, \vec{p})}^{\sigma}$, in which case the vector $\vec{p} \in \mathcal{D}_{L}$ should be identified with its image in $\mathcal{B}_{L}$ modulo vectors in $\Lambda_{L}^{*}$. If $n=1$, we introduce the one-point correlation as

$$
\widehat{K}_{\alpha}^{\beta, L}:=\frac{1}{\beta L^{2}}\left\langle\hat{J}_{\alpha, \mathbf{0}}\right\rangle_{\beta, L}
$$

Moreover, for $\mathbf{p}_{i} \in \mathbb{R}^{2}$, we let

$$
\widehat{K}_{\alpha_{1}, \ldots, \alpha_{n}}\left(\mathbf{p}_{1}, \ldots, \mathbf{p}_{n-1}\right):=\lim _{\beta \rightarrow \infty} \lim _{L \rightarrow \infty} \widehat{K}_{\alpha_{1}, \ldots, \alpha_{n}}^{\beta, L}\left(\mathbf{p}_{1}, \ldots, \mathbf{p}_{n-1}\right) .
$$

Note that these correlations are invariant under the exchange of the indices $\left(\alpha_{i}, \mathbf{p}_{i}\right)$ with $\left(\alpha_{j}, \mathbf{p}_{j}\right)$ (and, if either $i$ or $j$ are equal to $n, \mathbf{p}_{n}$ should be interpreted as $-\mathbf{p}_{2}-\cdots-\mathbf{p}_{n-1}$ ).

A crucial fact for the following is that the infinite volume and zero temperature correlations functions are analytic in $U$ and infinitely differentiable in the momenta, as summarized in the following proposition.

Proposition 4.1 [Existence and regularity of the interacting correlations] There exists $U_{0}>0$, independent of $\beta$ and $L$, such that, for $|U|<U_{0}$ and $\beta, L$ sufficiently large, the correlations $\widehat{K}_{\alpha_{1}, \ldots, \alpha_{n}}^{\beta, L}\left(\mathbf{p}_{1}, \ldots, \mathbf{p}_{n-1}\right)$ are analytic in $U$, uniformly in $\beta, L$ and in their arguments. Moreover, the infinite volume and zero temperature limits of the correlations in (4.3) exist, and define a sequence of functions $\widehat{K}_{\alpha_{1}, \ldots, \alpha_{n}}\left(\mathbf{p}_{1}, \ldots, \mathbf{p}_{n-1}\right)$ that are analytic in $U$ in $|U| \leq U_{0}$, and are $C^{\infty}$ in their arguments. 
The proof is given in section 5 . The $C^{\infty}$ regularity of $\widehat{K}_{\alpha_{1}, \ldots, \alpha_{n}}\left(\mathbf{p}_{1}, \ldots, \mathbf{p}_{n-1}\right)$ could be improved to analyticity, in the case that both $H^{(0)}(\vec{x})$ and $v(\vec{x})$ decay exponentially at large distances.

\section{B. Ward Identities}

The components of $\hat{J}_{\alpha, \mathbf{p}}$ with $\alpha=\mu \in\{0,1,2\}$ are related among each other by the continuity equation (3.8), which is an exact identity at finite volume and temperature. If plugged into the definition of correlation functions, this equation implies exact relations among the correlations, known as Ward Identities.

Proposition 4.2 [Ward Identities] Under the same hypotheses as proposition 4.1, if $n \geq$ 2 and $\mathbf{p}_{i}=\left(\omega_{i}, \vec{p}_{i}\right) \in(2 \pi / \beta) \mathbb{Z} \times \mathcal{D}_{L}, \forall i=1, \ldots, n-1$, the following identity holds:

$$
\sum_{\mu=0}^{2}(i)^{\delta_{\mu, 0}}\left(\mathbf{p}_{1}\right)_{\mu} \widehat{K}_{\mu, \alpha_{2}, \ldots, \alpha_{n}}^{\beta, L}\left(\mathbf{p}_{1}, \mathbf{p}_{2}, \ldots, \mathbf{p}_{n-1}\right)=\sum_{j=2}^{n} \widehat{S}_{\alpha_{j} ; \widehat{\alpha}_{j}}^{\beta, L}\left(\mathbf{p}_{1}, \ldots, \mathbf{p}_{n-1}\right)
$$

where $\underline{\widehat{\alpha}}_{j}$ denotes the sequence $\underline{\alpha}=\left(\alpha_{2}, \ldots, \alpha_{n}\right)$ with the element $\alpha_{j}$ removed, and

$$
\begin{aligned}
& \widehat{S}_{\alpha_{j} ; \underline{\alpha}_{j}}^{\beta, L}\left(\mathbf{p}_{1}, \mathbf{p}_{2}, \ldots, \mathbf{p}_{n-1}\right)= \\
& \quad=\frac{1}{\beta L^{2}}\left\langle\mathbf{T} \hat{\Delta}_{\alpha_{j}}\left(\mathbf{p}_{1}, \mathbf{p}_{j}\right) ; \hat{J}_{\alpha_{2}, \mathbf{p}_{2}} ; \cdots ; \hat{J}_{\alpha_{j-1}, \mathbf{p}_{j-1}} ; \hat{J}_{\alpha_{j+1}, \mathbf{p}_{j+1}} ; \cdots ; \hat{J}_{\alpha_{n},-\mathbf{p}_{1} \cdots-\mathbf{p}_{n-1}}\right\rangle_{\beta, L} .
\end{aligned}
$$

with

$$
\hat{\Delta}_{\alpha}\left(\mathbf{p}_{1}, \mathbf{p}_{2}\right)=\int_{0}^{\beta} d t e^{-i t\left(\omega_{1}+\omega_{2}\right)}\left[\tilde{J}_{0, \vec{p}_{1}}, \tilde{J}_{\alpha, \vec{p}_{2}}\right]_{t} .
$$

The identity (4.4) is also valid for the infinite volume and zero temperature limits of the correlation functions.

\section{Remarks.}

- In the right side of (4.5), if $j=n$, then the vector $\mathbf{p}_{n}$ in the argument of $\hat{\Delta}_{\alpha_{n}}$ should be interpreted as $-\mathbf{p}_{1}-\cdots-\mathbf{p}_{n-1}$. In (4.6), $\tilde{J}_{\alpha, \vec{p}}=\left.\tilde{J}_{\alpha,(t, \vec{p})}\right|_{t=0}$, and $\left[\tilde{J}_{0, \vec{p}_{1}}, \tilde{J}_{\alpha, \vec{p}_{2}}\right]_{t}$ denotes the imaginary time evolution of $\left[\tilde{J}_{0, \vec{p}_{1}}, \tilde{J}_{\alpha, \vec{p}_{2}}\right]$.

- The term in the right side of (4.4) is called the Schwinger term. It is, of course, absent if the observables $\tilde{J}_{0, \vec{p}_{1}}$ and $\tilde{J}_{\alpha, \vec{p}_{2}}$ commute, i.e., for $\alpha=0$ or $\alpha \in I$.

Proof of proposition 4.2. By integrating by parts with respect to $t_{1}$, we find (noting that the boundary terms cancel)

$$
i \omega_{1} \widehat{K}_{0, \underline{\alpha}}^{\beta, L}\left(\mathbf{p}_{1}, \ldots, \mathbf{p}_{n-1}\right)=\frac{1}{\beta L^{2}} \int_{0}^{\beta} d t_{1} e^{-i \omega_{1} t_{1}} \partial_{t_{1}}\left\langle\mathbf{T} \tilde{J}_{0,\left(t_{1}, \vec{p}_{1}\right)} ; \hat{J}_{\alpha_{2}, \mathbf{p}_{2}} ; \cdots ; \hat{J}_{\alpha_{n}, \mathbf{p}_{n}}\right\rangle_{\beta, L}
$$


where $\mathbf{p}_{n}=-\mathbf{p}_{1}-\cdots-\mathbf{p}_{n-1}$. Recalling the definition of time-ordered correlation function, we see that the derivative with respect to $t_{1}$ can act either on the observable $\tilde{J}_{0,\left(t_{1}, \vec{p}_{1}\right)}$, in which case we can apply the continuity equation (3.8), or on the characteristic functions entering the definition of time-ordering. Therefore, (4.7) can be rewritten as

$$
\begin{aligned}
& i \omega_{1} \widehat{K}_{0, \underline{\alpha}}^{\beta, L}\left(\mathbf{p}_{1}, \ldots, \mathbf{p}_{n-1}\right)=-\frac{1}{\beta L^{2}} \vec{p}_{1} \cdot\left\langle\mathbf{T} \overrightarrow{\hat{J}}_{\mathbf{p}_{1}} ; \hat{J}_{\alpha_{2}, \mathbf{p}_{2}} ; \cdots ; \hat{J}_{\alpha_{n}, \mathbf{p}_{n}}\right\rangle_{\beta, L}+\frac{1}{\beta L^{2}} \int_{0}^{\beta} d t_{1} \sum_{j=2}^{n} \times \\
& \times e^{-i\left(\omega_{1}+\omega_{j}\right) t_{1}}\left\langle\mathbf{T}\left[\tilde{J}_{0, \vec{p}_{1}} ; \hat{J}_{\alpha_{j}, \vec{p}_{j}}\right]_{t_{1}} ; \hat{J}_{\alpha_{2}, \mathbf{p}_{2}} ; \cdots ; \hat{J}_{\alpha_{j-1}, \mathbf{p}_{j-1}} ; \hat{J}_{\alpha_{j+1}, \mathbf{p}_{j+1}} ; \cdots ; \hat{J}_{\alpha_{n}, \mathbf{p}_{n}}\right\rangle_{\beta, L}
\end{aligned}
$$

where, for any collection of even observables $O^{(1)}, \ldots, O^{(n)}$,

$$
\begin{aligned}
\left\langle\mathbf{T}\left[O^{(1)} ; O^{(2)}\right]_{t_{1}} ; O_{t_{3}}^{(3)} ; \cdots ; O_{t_{n}}^{(n)}\right\rangle_{\beta, L} & =\left.\left\langle\mathbf{T} O_{t_{1}}^{(1)} ; O_{t_{2}}^{(2)} ; O_{t_{3}}^{(3)} ; \cdots ; O_{t_{n}}^{(n)}\right\rangle_{\beta, L}\right|_{t_{2}=t_{1}+0^{-}} \\
& -\left.\left\langle\mathbf{T} O_{t_{2}}^{(2)} ; O_{t_{1}}^{(1)} ; O_{t_{3}}^{(3)} ; \cdots ; O_{t_{n}}^{(n)}\right\rangle_{\beta, L}\right|_{t_{2}=t_{1}+0^{+}}
\end{aligned}
$$

Now, a straightforward implication of the definition of time-ordered truncated expectations is that

$$
\begin{aligned}
& \left\langle\mathbf{T} O_{t_{1}}^{(1)} ; O_{t_{2}}^{(2)} ; \cdots ; O_{t_{n}}^{(n)}\right\rangle_{\beta, L}=\left\langle\mathbf{T} O_{t_{1}}^{(1)} O_{t_{2}}^{(2)} ; \cdots ; O_{t_{n}}^{(n)}\right\rangle_{\beta, L} \\
& \quad-\sum_{\substack{\left\{i_{1}, \ldots, i_{p}\right\} \\
\left\{j_{1}, \ldots j_{q}\right\}}}^{*}\left\langle\mathbf{T} O_{t_{1}}^{(1)} ; O_{t_{i_{1}}}^{\left(i_{1}\right)} ; \cdots ; O_{t_{i_{p}}}^{\left(i_{p}\right)}\right\rangle_{\beta, L}\left\langle\mathbf{T} O_{t_{2}}^{(2)} ; O_{t_{j_{1}}}^{\left(j_{1}\right)} ; \cdots ; O_{t_{j_{q}}}^{\left(j_{q}\right)}\right\rangle_{\beta, L}
\end{aligned}
$$

where the sum in the second line is over all the partitions of $\{3, \ldots, n-1\}$ in two disjoint subsets, $\left\{i_{1}, \ldots, i_{p}\right\}$ and $\left\{j_{1}, \ldots, j_{q}\right\}$. By plugging this identity in the right side of (4.9), we obtain that

$$
\left\langle\mathbf{T}\left[O^{(1)} ; O^{(2)}\right]_{t_{1}} ; O_{t_{3}}^{(3)} ; \cdots ; O_{t_{n}}^{(n)}\right\rangle_{\beta, L}=\left\langle\mathbf{T}\left[O^{(1)}, O^{(2)}\right]_{t_{1}} ; O_{t_{3}}^{(3)} ; \cdots ; O_{t_{n}}^{(n)}\right\rangle_{\beta, L} .
$$

(Note the comma between $O_{t_{1}}^{(1)}$ and $O_{t_{2}}^{(2)}$ in the right side, instead of the semicolon). Finally, by using (4.11) in the second line of (4.8), we obtain (4.4). By taking the limit as the volume goes to infinity and the temperature to zero, and using the existence and analyticity of the limiting correlations stated in proposition 4.1, we obtain that (4.4) is also valid for the limiting correlations.

The Ward identities have important consequences on the momentum-dependence of the current-current correlations. The following corollary will play a crucial role in the proof of our main result.

Corollary 4.1 Under the same hypotheses as proposition 4.1, the infinite volume and zero temperature correlations satisfy the following identities:

(1) If $n \geq 2, j \in\{1,2\}$ and $\underline{\sigma}=\left(\sigma_{2}, \ldots, \sigma_{n}\right) \in I^{n-1}$,

$$
\widehat{K}_{j, \underline{\sigma}}\left((\omega, \overrightarrow{0}), \mathbf{p}_{2}, \ldots, \mathbf{p}_{n-1}\right)=-i \omega \frac{\partial \widehat{K}_{0, \underline{\sigma}}}{\partial p_{1, j}}\left((\omega, 0), \mathbf{p}_{2}, \ldots, \mathbf{p}_{n-1}\right) .
$$


(2) If $n \geq 3, j, j^{\prime} \in\{1,2\}$ and $\underline{\sigma}=\left(\sigma_{3}, \ldots, \sigma_{n}\right) \in I^{n-2}$,

$$
\begin{aligned}
\widehat{K}_{j, j^{\prime}, \underline{\sigma}}\left(\left(\omega_{1}, \overrightarrow{0}\right),\left(\omega_{2}, \overrightarrow{0}\right), \mathbf{p}_{3}, \ldots, \mathbf{p}_{n-1}\right) & -\frac{\partial \widehat{S}_{j^{\prime} ;}}{\partial p_{1, j}}\left(\left(\omega_{1}, \overrightarrow{0}\right),\left(\omega_{2}, \overrightarrow{0}\right), \mathbf{p}_{3}, \ldots, \mathbf{p}_{n-1}\right)= \\
= & -\omega_{1} \omega_{2} \frac{\partial^{2} \widehat{K}_{0,0, \underline{\sigma}}}{\partial p_{1, j} \partial p_{2, j^{\prime}}}\left(\left(\omega_{1}, \overrightarrow{0}\right),\left(\omega_{2}, \overrightarrow{0}\right), \mathbf{p}_{3}, \ldots, \mathbf{p}_{n-1}\right) .
\end{aligned}
$$

\section{Remarks.}

- These equations are just two special examples of relations among the correlations and their derivatives that can be obtained from the Ward Identities, by using the differentiability of the correlation functions stated in proposition 4.1. We limit ourselves to stating these two equations, because they are only the ones playing a role in the proof of our main result.

- Similar consequences of the Ward Identities have been used by Coleman and Hill [23] to prove that all the contributions to the topological mass of $\mathrm{QED}_{2+1}$ beyond one-loop vanish exactly.

Proof of corollary 4.1. In order to prove (4.12), consider the limit as $\beta, L \rightarrow \infty$ of (4.4) with $\left(\alpha_{2}, \ldots, \alpha_{n}\right)=\underline{\sigma}$, which reads

$$
i \omega_{1} \widehat{K}_{0, \underline{\sigma}}\left(\mathbf{p}_{1}, \ldots, \mathbf{p}_{n-1}\right)+\sum_{i=1}^{2} p_{1, i} \widehat{K}_{i, \underline{\sigma}}\left(\mathbf{p}_{1}, \ldots, \mathbf{p}_{n-1}\right)=0 .
$$

Recall that these correlations are differentiable, by proposition 4.1. Therefore, we can derive this equation with respect to $p_{1, j}$. If we do so, and then compute it at $\mathbf{p}_{1}=(\omega, \overrightarrow{0})$, we obtain (4.12).

In order to prove (4.13), let us proceed as follows. Consider the $\beta, L \rightarrow \infty$ of (4.4) with $\left(\alpha_{2}, \ldots, \alpha_{n}\right)=\left(j^{\prime}, \underline{\sigma}\right)$, which reads

$$
i \omega_{1} \widehat{K}_{0, j^{\prime}, \underline{\sigma}}\left(\mathbf{p}_{1}, \ldots, \mathbf{p}_{n-1}\right)+\sum_{i=1}^{2} p_{1, i} \widehat{K}_{i, j^{\prime}, \underline{\sigma}}\left(\mathbf{p}_{1}, \ldots, \mathbf{p}_{n-1}\right)=\widehat{S}_{j^{\prime} ; \underline{\sigma}}\left(\mathbf{p}_{1}, \ldots, \mathbf{p}_{n-1}\right) .
$$

By deriving it with respect to $p_{1, j}$, we obtain

$$
\begin{aligned}
& \widehat{K}_{j, j^{\prime}, \underline{\sigma}}\left(\mathbf{p}_{1}, \ldots, \mathbf{p}_{n-1}\right)-\frac{\partial}{\partial p_{1, j}} \widehat{S}_{j^{\prime} ; \underline{\sigma}}\left(\mathbf{p}_{1}, \ldots, \mathbf{p}_{n-1}\right)= \\
& =-i \omega_{1} \frac{\partial}{\partial p_{1, j}} \widehat{K}_{0, j^{\prime}, \underline{\sigma}}\left(\mathbf{p}_{1}, \ldots, \mathbf{p}_{n-1}\right)-\sum_{i=1}^{2} p_{1, i} \frac{\partial}{\partial p_{1, j}} \widehat{K}_{i, j^{\prime}, \underline{\sigma}}\left(\mathbf{p}_{1}, \ldots, \mathbf{p}_{n-1}\right)
\end{aligned}
$$

Similarly, consider $(4.4)$, with $\left(\alpha_{2}, \ldots, \alpha_{n}\right)=(0, \underline{\sigma})$. By using the invariance of $\widehat{K}_{\alpha_{1}, \ldots, \alpha_{n}}\left(\mathbf{p}_{1}, \ldots, \mathbf{p}_{n-1}\right)$ under the exchange of $\left(\alpha_{1}, \mathbf{p}_{1}\right)$ with $\left(\alpha_{2}, \mathbf{p}_{2}\right)$, we obtain

$$
i \omega_{2} \widehat{K}_{0,0, \underline{\sigma}}\left(\mathbf{p}_{1}, \ldots, \mathbf{p}_{n-1}\right)+\sum_{i=1}^{2} p_{2, i} \widehat{K}_{0, i, \underline{\sigma}}\left(\mathbf{p}_{1}, \ldots, \mathbf{p}_{n-1}\right)=0
$$


and by deriving this with respect to $p_{2, j^{\prime}}$, we find:

$$
\begin{aligned}
\widehat{K}_{0, j^{\prime}, \underline{\sigma}}\left(\mathbf{p}_{1}, \ldots, \mathbf{p}_{n-1}\right)= & -i \omega_{2} \frac{\partial}{\partial p_{2, j^{\prime}}} \widehat{K}_{0,0, \underline{\sigma}}\left(\mathbf{p}_{1}, \ldots, \mathbf{p}_{n-1}\right) \\
& -\sum_{i=1,2} p_{2, i} \frac{\partial}{\partial p_{2, j^{\prime}}} \widehat{K}_{0, i, \underline{\sigma}}\left(\mathbf{p}_{1}, \ldots, \mathbf{p}_{n-1}\right) .
\end{aligned}
$$

By plugging this equation into (4.16) and then setting $\mathbf{p}_{1}=\left(\omega_{1}, \overrightarrow{0}\right)$ and $\mathbf{p}_{2}=\left(\omega_{2}, \overrightarrow{0}\right)$, we obtain (4.13).

\section{Schwinger-Dyson equation}

In this section we derive an equation relating the two-point current-current correlation, which enters the definition of conductivity, with higher-point correlations, known as the Schwinger-Dyson equation. The equation will be expressed order by order in perturbation theory, which is not a limitation, since, in light of proposition 4.1, the correlations are analytic in $U$, if $|U| \leq U_{0}$.

The starting point is the convergent perturbative expansion of the current-current correlation. By using the Duhamel's formula, one can easily prove that

$$
\widehat{K}_{i, j}^{\beta, L}(\mathbf{p})=\sum_{k \geq 0}(-1)^{k} \frac{U^{k}}{k !} \widehat{K}_{i, j}^{\beta, L,(k)}(\mathbf{p}),
$$

where, if $\widetilde{\mathcal{V}}_{L}=\int_{0}^{\beta} d t\left(\mathcal{V}_{L}\right)_{t}$ is the integral of the imaginary-time evolution of the interaction,

$$
\widehat{K}_{i, j}^{\beta, L,(k)}(\mathbf{p})=\frac{1}{\beta L^{2}}\left\langle\mathbf{T} \hat{J}_{i, \mathbf{p}} ; \hat{J}_{j,-\mathbf{p}} ; \widetilde{\mathcal{V}}_{L}^{; k}\right\rangle_{\beta, L}^{(0)} .
$$

Here $\widetilde{\mathcal{V}}_{L}^{; k}$ is a shorthand notation for $\underbrace{\widetilde{\mathcal{V}}_{L} ; \widetilde{\mathcal{V}}_{L} ; \cdots ; \widetilde{\mathcal{V}}_{L}}_{k \text { times }}$, and the superscript ${ }^{(0)}$ is a shorthand for $\left.\right|_{U=0}$ : this means that both the Gibbs state and the time evolution of the operators in (4.20) are computed at $U=0$, i.e., with respect to the grand canonical Hamiltonian $\mathcal{H}_{L}^{(0)}-\mu \mathcal{N}_{L}$.

Note that $\widetilde{\mathcal{V}}_{L}$ can be conveniently rewritten in momentum space as

$$
\widetilde{\mathcal{V}}_{L}=\frac{1}{\beta L^{2}} \sum_{\mathbf{q} \in \frac{2 \pi}{\beta} \mathbb{Z} \times \mathcal{B}_{L}} \sum_{\sigma, \sigma^{\prime} \in I} \hat{n}_{\mathbf{q}}^{\sigma} \hat{\sigma}_{\sigma \sigma^{\prime}}(\vec{q}) \hat{n}_{-\mathbf{q}}^{\sigma^{\prime}}
$$

Now, plugging (4.21) in (4.20), we obtain that, for all $k \geq 1$,

$$
\widehat{K}_{i, j}^{\beta, L,(k)}(\mathbf{p})=\frac{1}{\left(\beta L^{2}\right)^{2}} \sum_{\mathbf{q} \in \frac{2 \pi}{\beta} \mathbb{Z} \times \mathcal{B}_{L}} \sum_{\sigma, \sigma^{\prime} \in I} \hat{v}_{\sigma \sigma^{\prime}}(\vec{q})\left\langle\mathbf{T} \hat{J}_{i, \mathbf{p}} ; \hat{J}_{j,-\mathbf{p}} ; \widetilde{\mathcal{V}}_{L}^{; k-1} ; \hat{n}_{\mathbf{q}}^{\sigma} \hat{n}_{-\mathbf{q}}^{\sigma^{\prime}}\right\rangle_{\beta, L}^{(0)}
$$


By using the combinatorial identity (4.10), we can further rewrite the average on the right side as

$$
\begin{aligned}
\langle\mathbf{T} & \left.\hat{J}_{i, \mathbf{p}} ; \hat{J}_{j,-\mathbf{p}} ; \widetilde{\mathcal{V}}_{L}^{k k-1} ; \hat{n}_{\mathbf{q}}^{\sigma} \hat{n}_{-\mathbf{q}}^{\sigma^{\prime}}\right\rangle_{\beta, L}^{(0)}=\left\langle\mathbf{T} \hat{J}_{i, \mathbf{p}} ; \hat{J}_{j,-\mathbf{p}} ; \widetilde{\mathcal{V}}_{L}^{; k-1} ; \hat{n}_{\mathbf{q}}^{\sigma} ; \hat{n}_{-\mathbf{q}}^{\sigma^{\prime}}\right\rangle_{\beta, L}^{(0)}+\sum_{m=0}^{k-1}\left(\begin{array}{c}
k-1 \\
m
\end{array}\right) \times \\
\times & {\left[\left\langle\mathbf{T} \hat{J}_{i, \mathbf{p}} ; \hat{J}_{j,-\mathbf{p}} ; \widetilde{\mathcal{V}}_{L}^{; m} ; \hat{n}_{\mathbf{q}}^{\sigma}\right\rangle_{\beta, L}^{(0)} \cdot\left\langle\mathbf{T} \widetilde{\mathcal{V}}_{L}^{; k-1-m} ; \hat{n}_{-\mathbf{q}}^{\left(\sigma^{\prime}\right)}\right\rangle_{\beta, L}^{(0)}+\left\langle\mathbf{T} \hat{J}_{i, \mathbf{p}} ; \widetilde{\mathcal{V}}_{L}^{; m} ; \hat{n}_{\mathbf{q}}^{\sigma}\right\rangle_{\beta, L}^{(0)} .\right.} \\
& \left.\cdot\left\langle\mathbf{T} \hat{J}_{j,-\mathbf{p}} ; \widetilde{\mathcal{V}}_{L}^{; k-1-m} ; \hat{n}_{-\mathbf{q}}^{\sigma^{\prime}}\right\rangle_{\beta, L}^{(0)}+\text { terms obtained by replacing } n_{\mathbf{q}}^{\sigma} \longleftrightarrow n_{-\mathbf{q}}^{\sigma^{\prime}}\right]
\end{aligned}
$$

The translation invariance of the Gibbs state implies that (denoting $\mathbf{q}=\left(\omega^{\prime}, \vec{q}\right)$ ):

$$
\begin{gathered}
\left\langle\mathbf{T} \hat{J}_{i, \mathbf{p}} ; \hat{J}_{j,-\mathbf{p}} ; \widetilde{\mathcal{V}}_{L}^{; m} ; \hat{n}_{\mathbf{q}}^{\sigma}\right\rangle_{\beta, L}^{(0)}=\delta_{\omega^{\prime}, 0} \delta_{\vec{q}, \overrightarrow{0}}\left\langle\mathbf{T} \hat{J}_{i, \mathbf{p}} ; \hat{J}_{j,-\mathbf{p}} ; \widetilde{\mathcal{V}}_{L}^{; m} ; \hat{n}_{\mathbf{0}}^{\sigma}\right\rangle_{\beta, L}^{(0)} \\
\left\langle\mathbf{T} \hat{J}_{i, \mathbf{p}} ; \widetilde{\mathcal{V}}_{L}^{; m} ; \hat{n}_{\mathbf{q}}^{\sigma}\right\rangle_{\beta, L}^{(0)}=\delta_{\omega^{\prime}+\omega, 0} \delta_{\vec{q}+\vec{p}, 0}\left\langle\mathbf{T} \hat{J}_{i, \mathbf{p}} ; \widetilde{\mathcal{V}}_{L}^{; m} ; \hat{n}_{-\mathbf{p}}^{\sigma}\right\rangle_{\beta, L}^{(0)} \\
\left\langle\mathbf{T} \widetilde{\mathcal{V}}_{L}^{; m} ; \hat{n}_{-\mathbf{q}}^{\sigma}\right\rangle_{\beta, L}^{(0)}=\delta_{\omega^{\prime}, 0} \delta_{\vec{q}, 0}\left\langle\mathbf{T} \widetilde{\mathcal{V}}_{L}^{; m} ; \hat{n}_{\mathbf{0}}^{\sigma}\right\rangle_{\beta, L}^{(0)}
\end{gathered}
$$

If we now substitute (4.23) and (4.24) into (4.22), we obtain the following remarkable identity, summarized here as a proposition.

Proposition 4.3 [Schwinger-Dyson equation] For all $k \geq 1, i, j \in\{1,2\}$, and $\mathbf{p} \in$ $\frac{2 \pi}{\beta} \mathbb{Z} \times \mathcal{D}_{L}$, the following identity holds:

$$
\begin{aligned}
\widehat{K}_{i, j}^{\beta, L,(k)}(\mathbf{p})= & \frac{1}{\beta L^{2}} \sum_{\mathbf{q} \in \frac{2 \pi}{\beta} \mathbb{Z} \times \mathcal{B}_{L}} \sum_{\sigma, \sigma^{\prime} \in I} \hat{v}_{\sigma \sigma^{\prime}}(\vec{q}) \widehat{K}_{i, j, \sigma, \sigma^{\prime}}^{\beta, L,(k-1)}(\mathbf{p},-\mathbf{p}, \mathbf{q}) \\
& +2 \sum_{m=0}^{k-1}\left(\begin{array}{c}
k-1 \\
m
\end{array}\right) \sum_{\sigma, \sigma^{\prime} \in I} \hat{v}_{\sigma \sigma^{\prime}}(\overrightarrow{0}) \widehat{K}_{i, j, \sigma}^{\beta, L,(m)}(\mathbf{p},-\mathbf{p}) \widehat{K}_{\sigma^{\prime}}^{\beta, L,(k-1-m)} \\
& +2 \sum_{m=0}^{k-1}\left(\begin{array}{c}
k-1 \\
m
\end{array}\right) \sum_{\sigma, \sigma^{\prime} \in I} \hat{v}_{\sigma \sigma^{\prime}}(-\vec{p}) \widehat{K}_{i, \sigma}^{\beta, L,(m)}(\mathbf{p}) \widehat{K}_{j, \sigma^{\prime}}^{\beta, L,(k-1-m)}(-\mathbf{p}) .
\end{aligned}
$$

Note that $\hat{v}_{\sigma \sigma^{\prime}}(-\vec{p})=\hat{v}_{\sigma^{\prime} \sigma}(\vec{p})$, and the argument of $\hat{v}_{\sigma \sigma^{\prime}}$ should be identified with its image in $\mathcal{B}_{L}$ modulo vectors in $\Lambda_{L}^{*}$.

\section{Proof of theorem 2.1}

We are finally in the position of proving our main result, theorem 2.1. The proof is based on a combination of the three results discussed in the previous subsections, namely: the analyticity of the correlation functions (proposition 4.1), the Ward identities (proposition 4.2 ), and the Schwinger-Dyson equation (proposition 4.3).

Proof of theorem 2.1. In light of theorem 3.1, it is enough to prove that $\bar{\sigma}_{i j}(U)=\bar{\sigma}_{i j}(0)$, for $U$ small enough. First of all, by the analyticity of the current correlations stated in 
proposition 4.1, we have that $\bar{\sigma}_{i j}(U)$ is analytic in $U$ as well, as long as $|U| \leq U_{0}$. In this domain, $\bar{\sigma}_{i j}(U)$ can be written in convergent perturbation series as

$$
\bar{\sigma}_{i j}(U)=\bar{\sigma}_{i j}^{(0)}+\sum_{k \geq 1}(-1)^{k} \frac{U^{k}}{k !} \bar{\sigma}_{i j}^{(k)}
$$

where

$$
\bar{\sigma}_{i j}^{(k)}=-\frac{1}{A} \lim _{\omega \rightarrow 0} \frac{\partial}{\partial \omega} \widehat{K}_{i j}^{(k)}(\omega, \overrightarrow{0}) .
$$

Since the series in (4.26) is convergent, in order to prove theorem 2.1 it sufficies to show that:

$$
\bar{\sigma}_{i j}^{(k)}=0, \quad \text { for all } k \geq 1 .
$$

This will be proved by showing that the derivative of $\widehat{K}_{i j}^{(k)}(\omega, \overrightarrow{0})$ with respect to $\omega$ vanishes linearly as $\omega \rightarrow 0$. To this aim, we plug the Schwinger-Dyson equation (4.25) into (4.27), thus getting, for all $k \geq 1, \bar{\sigma}_{i j}^{(k)}=\mathrm{I}^{(k)}+\mathrm{II}^{(k)}+\mathrm{III}^{(k)}$, with

$$
\begin{aligned}
\mathrm{I}^{(k)} & :=-\frac{1}{A} \lim _{\omega \rightarrow 0} \int_{\mathbb{R} \times \mathcal{B}} \frac{d \mathbf{q}}{(2 \pi)|\mathcal{B}|} \sum_{\sigma, \sigma^{\prime} \in I} \hat{v}_{\sigma \sigma^{\prime}}(\vec{q}) \frac{\partial}{\partial \omega} \widehat{K}_{i, j, \sigma, \sigma^{\prime}}^{(k-1)}((\omega, \overrightarrow{0}),(-\omega, \overrightarrow{0}), \mathbf{q}) \\
\mathrm{II}^{(k)} & :=-\frac{2}{A} \lim _{\omega \rightarrow 0} \sum_{m=0}^{k-1}\left(\begin{array}{c}
k-1 \\
m
\end{array}\right) \sum_{\sigma, \sigma^{\prime} \in I} \hat{v}_{\sigma \sigma^{\prime}}(\overrightarrow{0}) \frac{\partial}{\partial \omega} \widehat{K}_{i, j, \sigma}^{(m)}((\omega, \overrightarrow{0}),(-\omega, \overrightarrow{0})) \widehat{K}_{\sigma^{\prime}}^{(k-1-m)} \\
\mathrm{III}^{(k)} & :=-\frac{2}{A} \lim _{\omega \rightarrow 0} \sum_{m=0}^{k-1}\left(\begin{array}{c}
k-1 \\
m
\end{array}\right) \sum_{\sigma, \sigma^{\prime} \in I} \hat{v}_{\sigma \sigma^{\prime}}(\overrightarrow{0}) \frac{\partial}{\partial \omega}\left[\widehat{K}_{i, \sigma}^{(m)}(\omega, \overrightarrow{0}) \widehat{K}_{j, \sigma^{\prime}}^{(k-1-m)}(-\omega, \overrightarrow{0})\right] .
\end{aligned}
$$

Now, by using corollary 4.1, we prove that the three contributions are separately zero.

The contribution $\mathrm{I}^{(k)}$. First of all, note that

$\frac{\partial}{\partial \omega} \widehat{K}_{i, j, \sigma, \sigma^{\prime}}^{(k-1)}((\omega, \overrightarrow{0}),(-\omega, \overrightarrow{0}), \mathbf{q})=\frac{\partial}{\partial \omega}\left[\widehat{K}_{i, j, \sigma, \sigma^{\prime}}^{(k-1)}((\omega, \overrightarrow{0}),(-\omega, \overrightarrow{0}), \mathbf{q})-\frac{\partial \widehat{S}_{j ; \sigma, \sigma^{\prime}}^{(k-1)}}{\partial p_{1, i}}((\omega, \overrightarrow{0}),(-\omega, \overrightarrow{0}), \mathbf{q})\right]$,

simply because, by the very definition (4.5)-(4.6) of $\widehat{S}_{j ; \sigma, \sigma^{\prime}}\left(\mathbf{p}_{1}, \mathbf{p}_{2}, \mathbf{p}_{3}\right)$, this function depends on $\omega_{1}$ and $\omega_{2}$ only upon the combination $\omega_{1}+\omega_{2}$, so that, in particular, $\widehat{S}_{j ; \sigma, \sigma^{\prime}}((\omega, \overrightarrow{0}),(-\omega, \overrightarrow{0}), \mathbf{q})$ is independent of $\omega$. We can now use (4.13), understood as an order by order identity between convergent power series in $U$, and we thus obtain

$$
\begin{aligned}
\mathrm{I}^{(k)} & =-\frac{1}{A} \lim _{\omega \rightarrow 0} \int_{\mathbb{R} \times \mathcal{B}} \frac{d \mathbf{q}}{(2 \pi)|\mathcal{B}|} \sum_{\sigma, \sigma^{\prime} \in I} \hat{v}_{\sigma \sigma^{\prime}}(\vec{q}) \frac{\partial}{\partial \omega}\left[\omega^{2} \frac{\partial^{2} \widehat{K}_{0,0, \sigma, \sigma^{\prime}}^{(k-1)}}{\partial p_{1, i} \partial p_{2, j}}((\omega, \overrightarrow{0}),(-\omega, \overrightarrow{0}), \mathbf{q})\right] \\
& =-\frac{1}{A} \lim _{\omega \rightarrow 0} \int_{\mathcal{B}} \frac{d \vec{q}}{|\mathcal{B}|} \sum_{\sigma, \sigma^{\prime} \in I} \hat{v}_{\sigma \sigma^{\prime}}(\vec{q})\left[2 \omega F_{1}^{(k-1)}(\omega, \vec{q})+\omega^{2} F_{2}^{(k-1)}(\omega, \vec{q})\right],
\end{aligned}
$$

where

$$
\begin{aligned}
& F_{1}^{(k-1)}(\omega, \vec{q})=\int_{\mathbb{R}} \frac{d \omega^{\prime}}{2 \pi} \frac{\partial^{2} \widehat{K}_{0,0, \sigma, \sigma^{\prime}}^{(k-1)}}{\partial p_{1, i} \partial p_{2, j}}\left((\omega, \overrightarrow{0}),(-\omega, \overrightarrow{0}),\left(\omega^{\prime}, \vec{q}\right)\right), \\
& F_{2}^{(k-1)}(\omega, \vec{q})=\int_{\mathbb{R}} \frac{d \omega^{\prime}}{2 \pi}\left[\frac{\partial^{3} \widehat{K}_{0,0, \sigma, \sigma^{\prime}}^{(k-1)}}{\partial \omega_{1} \partial p_{1, i} \partial p_{2, j}}-\frac{\partial^{3} \widehat{K}_{0,0, \sigma, \sigma^{\prime}}^{(k-1)}}{\partial \omega_{2} \partial p_{1, i} \partial p_{2, j}}\right]\left((\omega, \overrightarrow{0}),(-\omega, \overrightarrow{0}),\left(\omega^{\prime}, \vec{q}\right)\right) .
\end{aligned}
$$


Now, the key remark is that $F_{1}^{(k-1)}(\omega, \vec{q})$ and $F_{2}^{(k-1)}(\omega, \vec{q})$ are bounded uniformly in $\omega$ and $\vec{q}$, for all $i=1,2,3$, which immediately implies that the second line of (4.31) is zero, as desired. In order to prove that $\left|F_{i}^{(k-1)}(\omega, \vec{q})\right| \leq C$, uniformly in $\omega$ and $\vec{q}$, we rewrite

$$
\begin{aligned}
& \widehat{K}_{0,0, \sigma, \sigma^{\prime}}^{(k-1)}\left(\mathbf{p}_{1}, \mathbf{p}_{2}, \mathbf{p}_{3}\right)=\sum_{\underline{\sigma}} \int d \underline{t} \sum_{\underline{\vec{x}}} e^{-i \omega_{1} t-i \omega_{2} t^{\prime}-i \omega_{3} t^{\prime \prime}} e^{-i \overrightarrow{p_{1}} \vec{x}-i \overrightarrow{p_{2}} \vec{y}-i \overrightarrow{p_{3}} \vec{z}}\left[\prod_{l=1}^{k-1} v_{\sigma_{l}, \sigma_{l}^{\prime}}\left(\vec{x}_{l}-\vec{y}_{l}\right)\right] \times \\
& \quad \times\left\langle n_{(t, \vec{x})}^{\sigma_{0}} ; n_{\left(t^{\prime}, \vec{y}\right)}^{\sigma^{\prime}} ; n_{\left(t^{\prime}, \vec{z}\right)}^{\sigma} ; n_{\mathbf{0}}^{\sigma^{\prime}} ; n_{\left(t_{1}, \vec{x}_{1}\right)}^{\sigma_{1}} n_{\left(t_{1}, \vec{y}_{1}\right)}^{\sigma_{1}^{\prime}} ; \cdots ; n_{\left(t_{k-1}, \vec{x}_{k-1}\right)}^{\sigma_{k-1}} n_{\left(t_{k-1}, \vec{y}_{k-1}\right)}^{\sigma_{k-1}^{\prime}}\right)^{(0)}
\end{aligned}
$$

where: (i) $\underline{\sigma}$ is a shorthand for $\left(\sigma_{0}, \sigma_{0}^{\prime}, \sigma_{1}, \sigma_{1}^{\prime}, \ldots, \sigma_{k-1}, \sigma_{k-1}^{\prime}\right)$, and is summed over $I^{2 k}$, (ii) $\underline{t}$ is a shorthand for $\left(t, t^{\prime}, t^{\prime \prime}, t_{1}, \ldots, t_{k-1}\right)$, and is integrated over $\mathbb{R}^{k+2}$, (iii) $\underline{\vec{x}}$ is a shorthand for $\left(\vec{x}, \vec{y}, \vec{z}, \vec{x}_{1}, \vec{y}_{1}, \ldots, \vec{x}_{k-1}, \vec{y}_{k-1}\right)$, and is summed over $\Lambda^{2 k+1}$, where $\Lambda=\left\{\vec{x}: \vec{x}=n_{1} \vec{\ell}_{1}+\right.$ $\left.n_{2} \vec{\ell}_{2}, n_{i} \in \mathbb{Z}\right\}$, (iv) $\langle\cdot\rangle^{(0)}$ stands for $\lim _{\beta \rightarrow \infty} \lim _{L \rightarrow \infty}\langle\cdot\rangle_{\beta, L}^{(0)}$. By using (4.33), we can rewrite $F_{1}^{(k-1)}$ as

$$
\begin{aligned}
& F_{1}^{(k-1)}(\omega, \vec{q})=-\sum_{\underline{\sigma}} \int d \underline{\underline{t}} \sum_{\underline{\underline{x}}} e^{-i \omega\left(t-t^{\prime}\right)} e^{-i \vec{q} \vec{z}}(\vec{x})_{i}(\vec{y})_{j}\left[\prod_{l=1}^{k-1} v_{\sigma_{l}, \sigma_{l}}\left(\vec{x}_{l}-\vec{y}_{l}\right)\right] \times \\
& \quad \times\left\langle n_{(t, \vec{x})}^{\sigma_{0}} ; n_{\left(t^{\prime}, \vec{y}\right)}^{\sigma_{0}^{\prime}} ; n_{(0, \vec{z})}^{\sigma} ; n_{\mathbf{0}}^{\sigma^{\prime}} ; n_{\left(t_{1}, \vec{x}_{1}\right)}^{\sigma_{1}} n_{\left(t_{1}, \vec{y}_{1}\right)}^{\sigma_{1}^{\prime}} ; \cdots ; n_{\left(t_{k-1}, \vec{x}_{k-1}\right)}^{\sigma_{k-1}} n_{\left(t_{k-1}, \vec{y}_{k-1}\right)}^{\sigma_{k-1}^{\prime}}\right\rangle^{(0)} .
\end{aligned}
$$

The truncated expectation value of the number operators in the second line can be computed via the Wick rule, which is the following. Write each number operator in the form $n_{(s, \vec{w})}^{\rho}=$ $\psi_{(s, \vec{w}), \rho}^{+} \psi_{(s, \vec{w}), \rho}^{-}$, and consider all the possible pairings of the creation/annihilation operators such that each annihilation operator $\psi_{(s, \vec{w}), \rho}^{-}$is paired with a creation operator $\psi_{\left(s^{\prime}, \vec{w}^{\prime}\right), \rho^{\prime}}^{+}$, with the additional constraint that the resulting pairing $p$ is "connected" in the following sense. Consider the directed graph $G_{p}=\left(V, E_{p}\right)$ whose vertex set is $V=\left\{\vec{x}, \vec{y}, \vec{z}, \overrightarrow{0}, \vec{x}_{1}, \vec{y}_{1}, \ldots, \vec{y}_{k-1}\right\}$, and whose edge set $E_{p}$ consists of the ordered pairs $\left(\vec{x}_{l}, \vec{y}_{l}\right), l=1, \ldots, k-1$, as well as of the ordered pairs $\left(\vec{w}, \vec{w}^{\prime}\right)$ associated with the elements $\ell=\left(\psi_{(s, \vec{w}), \rho}^{-}, \psi_{\left(s^{\prime}, \vec{w}^{\prime}\right), \rho^{\prime}}^{+}\right)$of the pairing $p$ : we shall say that the pairing $p$ is connected if the graph $G_{p}$ is connected. Then associate each connected pairing $p$ with a value, given by the sign $\alpha_{p}$ of the permutation required to move every creation operator to the immediate right of the annihilation operator it is paired with, times the product over the pairs of the corresponding propagators, where the propagator corresponding to the pair $\ell=\left(\psi_{(s, \vec{w}), \rho}^{-}, \psi_{\left(s^{\prime}, \vec{w}^{\prime}\right), \rho^{\prime}}^{+}\right)$is

$$
\begin{aligned}
& g_{\ell} \equiv g_{\rho, \rho^{\prime}}\left(s-s^{\prime}, \vec{w}-\vec{w}^{\prime}\right)=\lim _{\beta \rightarrow \infty L \rightarrow \infty} \lim _{L \rightarrow}\left\langle\left.\mathbf{T} \psi_{(s, \vec{w}), \rho}^{-} \psi_{\left(s^{\prime}, \vec{w}^{\prime}\right), \rho^{\prime}}^{+}\right|_{\beta, L} ^{(0)}=\right. \\
& =\int_{\mathcal{B}} \frac{d \vec{k}}{|\mathcal{B}|} e^{-i \vec{k}\left(\vec{w}-\vec{w}^{\prime}\right)}\left[e^{-\left(s-s^{\prime}\right)\left(\hat{H}^{(0)}(\vec{k})-\mu\right)}\left(\mathbb{1}\left(s>s^{\prime}\right) P_{+}(\vec{k})-\mathbb{1}\left(s \leq s^{\prime}\right) P_{-}(\vec{k})\right)\right]_{\rho, \rho^{\prime}},
\end{aligned}
$$

where $P_{-}(\vec{k})$ is the projector over the filled bands (see the lines after $(2.13)$ ), and $P_{+}(\vec{k})=$ $\mathbb{1}-P_{-}(\vec{k})$. As already observed after $(2.13)$, under the gap condition, $P_{-}(\vec{k})$ is infinitely differentiable in $\vec{k}$. Therefore, $g_{\sigma \sigma^{\prime}}\left(s-s^{\prime}, \vec{w}-\vec{w}^{\prime}\right)$ decays exponentially in $s-s^{\prime}$ and faster than any power in $\vec{w}-\vec{w}^{\prime}$. For later convenience, if $\ell=\left(\psi_{(s, \vec{w}), \rho}^{-}, \psi_{\left(s^{\prime}, \vec{w}^{\prime}\right), \rho^{\prime}}^{+}\right)$, we denote by $\Delta \mathbf{x}_{\ell}$ the space-time difference associated with $\ell$, namely $\Delta \mathbf{x}_{\ell}=\left(\Delta t_{\ell}, \Delta \vec{x}_{\ell}\right)=\left(s-s^{\prime}, \vec{w}-\vec{w}^{\prime}\right)$. 
On the basis of the Wick rule explained above, (4.34) can be rewritten as

$$
F_{1}^{(k-1)}(\omega, \vec{q})=-\sum_{\underline{\sigma}} \int d \underline{t} \sum_{\underline{\underline{x}}} e^{-i \omega\left(t-t^{\prime}\right)} e^{-i \vec{q} \vec{z}}(\vec{x})_{i}(\vec{y})_{j}\left[\prod_{l=1}^{k-1} v_{\sigma_{l}, \sigma_{l}^{\prime}}\left(\vec{x}_{l}-\vec{y}_{l}\right)\right] \sum_{p \in \mathcal{G}_{c}} \alpha_{p} \prod_{\ell \in p} g_{\ell},
$$

where $\mathcal{G}_{c}$ is the set of connected pairings. In order to bound this expression, for each pairing $p$ we arbitrarily choose a connected tree subgraph of $G_{p}$, denoted by $T_{p}$, consisting of all the pairs $\left(\vec{x}_{l}, \vec{y}_{l}\right)$, with $l=1, \ldots, k-1$, and of other $k+2$ edges of $G_{p}$. With some abuse of notation, we shall denote by $T_{p}$ also the subset of $p$ whose pairs are graphically associated with edges of $T_{p}$. Next, we decompose $(\vec{x})_{i}$ along the path $\mathcal{C}_{p}^{\vec{x} \rightarrow \overrightarrow{0}}$ on $T_{p}$ from $\vec{x}$ to $\overrightarrow{0}$. By walking along the path $\mathcal{C}_{p}^{\vec{x} \rightarrow \overrightarrow{0}}$ from $\vec{x}$ to $\overrightarrow{0}$, some of the edges $e \in \mathcal{C}_{p}^{\vec{x} \rightarrow \overrightarrow{0}}$ may be oriented in the same direction as the walk, in which case we set $\alpha_{e}=+1$, and some others in the opposite direction, in which case we set $\alpha_{e}=-1$. We can then rewrite $(\vec{x})_{i}=\sum_{e \in \mathcal{C}_{p}^{\vec{x} \rightarrow \overrightarrow{0}}} \alpha_{e}\left(\Delta \vec{x}_{\ell_{e}}\right)_{i}$, where $\ell_{e}$ is the pair graphically associated with $e$. We use a similar decomposition for $(\vec{y})_{j}$.

In terms of these definitions, we can finally bound (4.36) as

$$
\left|F_{1}^{(k-1)}(\omega, \vec{q})\right| \leq(2 k+1)^{2} \sum_{\underline{\sigma}} \sum_{p \in \mathcal{G}_{c}}\left[\prod_{l=1}^{k-1}\|v\|_{1,2}\right]\left[\prod_{\ell \in p \cap T_{p}}\left\|g_{\ell}\right\|_{1,2}\right]\left[\prod_{\ell \in p \backslash T_{p}}\left\|g_{\ell}\right\|_{\infty}\right],
$$

where $\|v\|_{1, m}=\sup _{\sigma, \sigma^{\prime}} \sum_{\vec{x} \in \Lambda}|\vec{x}|^{m}\left|v_{\sigma, \sigma^{\prime}}(\vec{x})\right|,\left\|g_{\ell}\right\|_{1, m}=\sup _{\sigma, \sigma^{\prime}} \int_{\mathbb{R}} d t \sum_{\vec{x} \in \Lambda}|\vec{x}|^{m}\left|g_{\sigma, \sigma^{\prime}}(t, \vec{x})\right|$, and $\left\|g_{\ell}\right\|_{\infty}=\sup _{\sigma, \sigma^{\prime}} \sup _{t, \vec{x}}\left|g_{\sigma, \sigma^{\prime}}(t, \vec{x})\right|$. Now, using the fact that $v$ and $g$ decay to zero at large distances faster than any power, as well as the fact that number of terms in the sums over $\underline{\sigma}$ and over $p \in \mathcal{G}_{p}$ are bounded, respectively, by $|I|^{2 k}$ and by $(2 k+2)$ !, we obtain that

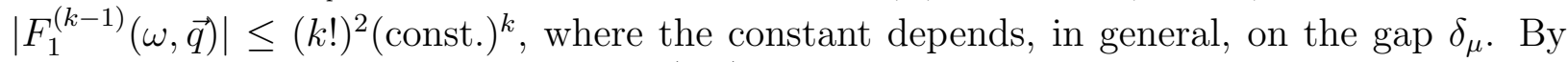
proceeding analogously, we see that $F_{2}^{(k-1)}$ can be bounded exactly in the same way. This concludes the proof of the uniform boundedness of $F_{i}^{(k-1)}$ and, as observed after (4.32), of the fact that $\mathrm{I}^{(k)}=0$.

Remark. The $(k !)^{2}$ dependence in the bounds on $F_{1}^{(k-1)}$ and $F_{2}^{(k-1)}$ naively suggests that the $k$-th order coefficient in the expansion (4.26) behaves like $\sim k$ ! at large $k$ (note the extra $1 / k$ ! in the right side of (4.26)), which seems incompatible with the stated analyticity of $\bar{\sigma}_{i j}(U)$. In fact, there is a better way of bounding the $k$-th order coefficient of the series, which is smaller by a factor $\sim k$ !, as compared to the bound presented above, which implies the analyticity of the series and will be discussed in the next section.

The contributions $\mathrm{II}^{(k)}$ and $\mathrm{III}^{(k)}$. The proof of the fact that $\mathrm{II}^{(k)}$ and $\mathrm{III}^{(k)}$ are zero goes along the same lines as the proof that $\mathrm{I}^{(k)}=0$. By using the independence of $\widehat{S}_{j ; \sigma}\left(\left(\omega, \vec{p}_{1}\right),\left(-\omega, \vec{p}_{2}\right)\right)$ on $\omega$ and the identity (4.13), we find that

$$
\begin{aligned}
\frac{\partial}{\partial \omega} \widehat{K}_{i, j, \sigma}^{(m)}((\omega, \overrightarrow{0}),(-\omega, \overrightarrow{0})) & =\frac{\partial}{\partial \omega}\left[\widehat{K}_{i, j, \sigma}^{(m)}((\omega, \overrightarrow{0}),(-\omega, \overrightarrow{0}))-\frac{\partial \widehat{S}_{j ; \sigma}^{(m)}}{\partial p_{1, i}}((\omega, \overrightarrow{0}),(-\omega, \overrightarrow{0}))\right] \\
& =\frac{\partial}{\partial \omega}\left[\omega^{2} \frac{\partial^{2} \widehat{K}_{0,0, \underline{\sigma}}^{(m)}}{\partial p_{1, j} \partial p_{2, j^{\prime}}}((\omega, \overrightarrow{0}),(-\omega, \overrightarrow{0}))\right],
\end{aligned}
$$


so that

$$
\mathrm{II}^{(k)}=-\frac{2}{A} \lim _{\omega \rightarrow 0} \sum_{\sigma, \sigma^{\prime} \in I} \hat{v}_{\sigma \sigma^{\prime}}(\overrightarrow{0})\left[2 \omega F_{3}^{(k-1)}(\omega)+\omega^{2} F_{4}^{(k-1)}(\omega)\right],
$$

where

$$
\begin{aligned}
F_{3}^{(k-1)}(\omega) & =\sum_{\substack{m_{1}, m_{2}: \\
m_{1}+m_{2}=k-1}}\left(\begin{array}{c}
k-1 \\
m_{1}
\end{array}\right) \frac{\partial^{2} \widehat{K}_{0,0, \underline{\sigma}}^{\left(m_{1}\right)}}{\partial p_{1, j} \partial p_{2, j^{\prime}}}((\omega, \overrightarrow{0}),(-\omega, \overrightarrow{0})) \cdot \widehat{K}_{\sigma^{\prime}}^{\left(m_{2}\right)} \\
F_{4}^{(k-1)}(\omega) & =\sum_{\substack{m_{1}, m_{2}: \\
m_{1}+m_{2}=k-1}}\left(\begin{array}{c}
k-1 \\
m_{1}
\end{array}\right)\left[\frac{\partial^{3} \widehat{K}_{0,0, \underline{\sigma}}^{\left(m_{1}\right)}}{\partial \omega_{1} \partial p_{1, j} \partial p_{2, j^{\prime}}}-\frac{\partial^{3} \widehat{K}_{0,0, \underline{\sigma}}^{\left(m_{1}\right)}}{\partial \omega_{2} \partial p_{1, j} \partial p_{2, j^{\prime}}}\right]((\omega, \overrightarrow{0}),(-\omega, \overrightarrow{0})) \cdot \widehat{K}_{\sigma^{\prime}}^{\left(m_{2}\right)} .
\end{aligned}
$$

Similarly, using (4.12), we can rewrite

$$
\widehat{K}_{i, \sigma}^{\left(m_{1}\right)}(\omega, \overrightarrow{0})=-i \omega \frac{\partial \widehat{K}_{0, \sigma}^{\left(m_{1}\right)}}{\partial p_{i}}(\omega, \overrightarrow{0}), \quad \widehat{K}_{j, \sigma^{\prime}}^{\left(m_{2}\right)}(-\omega, \overrightarrow{0})=i \omega \frac{\partial \widehat{K}_{0, \sigma^{\prime}}^{\left(m_{2}\right)}}{\partial p_{j}}(-\omega, \overrightarrow{0}),
$$

so that

$$
\mathrm{III}^{(k)}=-\frac{2}{A} \lim _{\omega \rightarrow 0} \sum_{\sigma, \sigma^{\prime} \in I} \hat{v}_{\sigma \sigma^{\prime}}(\overrightarrow{0})\left[2 \omega F_{5}^{(k-1)}(\omega)+\omega^{2} F_{6}^{(k-1)}(\omega)\right],
$$

where

$$
\begin{aligned}
F_{5}^{(k-1)}(\omega) & =\sum_{\substack{m_{1}, m_{2}: \\
m_{1}+m_{2}=k-1}}\left(\begin{array}{c}
k-1 \\
m_{1}
\end{array}\right) \frac{\partial \widehat{K}_{0, \sigma}^{\left(m_{1}\right)}}{\partial p_{i}}(\omega, \overrightarrow{0}) \frac{\partial \widehat{K}_{0, \sigma^{\prime}}^{\left(m_{2}\right)}}{\partial p_{j}}(-\omega, \overrightarrow{0}), \\
F_{6}^{(k-1)}(\omega) & =\sum_{\substack{m_{1}, m_{2}: \\
m_{1}+m_{2}=k-1}}\left(\begin{array}{c}
k-1 \\
m_{1}
\end{array}\right)\left[\frac{\partial^{2} \widehat{K}_{0, \sigma}^{\left(m_{1}\right)}}{\partial \omega \partial p_{i}}(\omega, \overrightarrow{0}) \frac{\partial \widehat{K}_{0, \sigma^{\prime}}^{\left(m_{2}\right)}}{\partial p_{j}}(-\omega, \overrightarrow{0})-\frac{\partial \widehat{K}_{0, \sigma}^{\left(m_{1}\right)}}{\partial p_{i}}(\omega, \overrightarrow{0}) \frac{\partial^{2} \widehat{K}_{0, \sigma^{\prime}}^{\left(m_{2}\right)}}{\partial \omega \partial p_{j}}(-\omega, \overrightarrow{0})\right] .
\end{aligned}
$$

By proceeding as in the proof of (4.37), one obtains that $F_{i}^{(k-1)}(\omega)$ are bounded uniformly in $\omega$, which implies that $\mathrm{II}^{(k)}=\mathrm{III}^{(k)}=0$, as desired. This concludes the proof of $(4.28)$, and of theorem 2.1 .

\section{ANALYTICITY}

In this section we prove proposition 4.1, concerning the analyticity in $U$ and the smoothness in $\mathbf{p}$ of the multi-point current/density correlation functions. Roughly, the strategy will consist in: (i) reformulating the correlation functions in terms of a Grassmann integral, in the limit where a suitable cutoff function is removed; (ii) proving the analyticity of the Grassmann integral, uniformly in the cutoff parameter; (iii) using Vitali's theorem on the convergence of holomorphic functions (also known as Vitali-Porter theorem, or Weierstrass' theorem), to conclude that the correlations themselves are analytic. The analysis of this section is a straightforward adaptation of previous works, see, e.g., [34, Appendices 
B,C,D] or [33, Section 6] for two recent reviews in the context of graphene with short-range interactions, and is included here just for the sake of self- containedness.

As we shall see, our proof of analyticity uses a multiscale analysis, which seems like overkill in a situation like ours where the propagator decays faster than any power in space and time, uniformly in $\beta$ and $L$. Before we delve into the proof, which is quite technical, let us then explain why a naive single-scale approach fails, and what are the main ideas that led us to use a multiscale analysis.

The generic order in perturbation theory can be expressed as a sum over connected pairings, in complete analogy with the representation of the second line of (4.34) discussed after that equation. Each pairing is associated with a value, which is bounded uniformly in the parameters involved, in analogy with (4.37). However, after summing over the possible pairings, the bound on the $k$-th order coefficient scales like $C^{k} k$ !, where $k$ ! should be thought of as the product of the factor $1 / k$ ! appearing in the Taylor expansion (cf., e.g., with (4.26)) times the number of possible pairings, which grows like $(k !)^{2}$, see the lines after (4.37). In other words, if we bound pairing by pairing the contributions to the $k$-th order in perturbation theory, we get a contribution that is not summable over $k$, because it is off by a combinatorial factor $\sim k$ !.

This problem is reminiscent of the problem of convergence of the virial (low-density/hightemperature) expansion in classical statistical mechanics, where the $k$-th order coefficient is the sum of several contributions, each of which is easily seen to be bounded. However, the number of contributions to the $k$-th order of the virial expansion is too large $\left(\sim C^{k^{2}}\right)$ and, therefore, the convergence of the series requires the exhibition of cancellations among the various contributions.

In the fermionic problem at hand, the required cancellations arise from the fermionic statistics: the $k$-th order coefficient in perturbation theory can be expressed in the form of a determinant, whose norm is in many situations much smaller than the sum of the norms of the single contributions to the determinant. For instance, if the generic element of the matrix $A$ is expressible in the form of a scalar product, $A_{i j}=\left(u_{i}, v_{j}\right)$, then $\operatorname{det} A$ can be conveniently bounded (via the so-called Gram-Hadamard inequality) as: $|\operatorname{det} A| \leq$ $\prod_{i}\left\|u_{i}\right\| \cdot\left\|v_{i}\right\|$, where $\|\cdot\|$ is the norm induced by the scalar product. Such a bound is free of bad factorials and seems to make the job. Unfortunately, in the case at hand, the elements of the matrix of interest are propagators $g_{\sigma_{i} \sigma_{j}}\left(t_{i}-t_{j}, \vec{x}_{i}-\vec{x}_{j}\right)$, which are not expressible as the scalar product of two vectors on any separable Hilbert space [51], due to a jump singularity in the time dependence of the propagator. The jump singularity is induced by the time ordering arising from the Duhamel's formula. There are several ways out of this problem. One is to expand the determinants in the form of "chronologically ordered" determinants, each of which is free from jump singularities and can be bounded by the Gram-Hadamard inequality, as in [51]. This approach has the advantage that it allows to obtain constructive bounds without any multi-scale analysis, but in order to do so, it is crucial that the free propagator decays sufficiently fast (as it is the case, e.g., in gapped systems). Recently, 
it has been shown that single-scale constructive bounds can also be obtained without the use of chronologically ordered determinants, via non-commutative Hölder inequalities [20]. Another possibility is to impose an ultraviolet cutoff on the imaginary frequencies, and to re-express the regularized propagator as the sum of single-scale propagators, each admitting a scalar product representation, in terms of vectors of uniformly bounded norm. In this way, we get rid of the combinatorial factor related to the $k$ ! explained above, at the cost of analyzing a simple multiscale problem. Even if slightly more technical than the first method, this approach has the advantage of being adaptable to massless situations, with propagator decaying slowly (in a non-integrable way) at large distances, such as those studied in [1114, 34-36]. We believe that, by applying the multiscale methods of these papers, our theorem 2.1 could be extended arbitrarily close to the massless line $\delta_{\mu}=0$. In this perspective, we prefer to present here a multiscale proof of the analyticity of the correlations, and we plan to come back to the problem of extending it to the infrared regime in a future publication.

\section{A. Grassmann representation}

Let us preliminarily recall a few known facts about perturbation theory for the free energy and correlations of interacting fermionic systems, which we need for justifying their Grassmann representation. We first discuss the free energy, which is simpler. Using Duhamel's expansion, we can rewrite the (a priori formal) series expansion of the interacting partition function in the parameter $U$ as:

$$
\frac{\operatorname{Tr}_{\mathcal{F}} e^{-\beta\left(\mathcal{H}_{L}-\mu \mathcal{N}_{L}\right)}}{\operatorname{Tr}_{\mathcal{F}} e^{-\beta\left(\mathcal{H}_{L}^{(0)}-\mu \mathcal{N}_{L}\right)}}=1+\sum_{n \geq 1}(-U)^{n} \int_{0}^{\beta} d t_{1} \cdots \int_{0}^{t_{n-1}} d t_{n} \frac{\operatorname{Tr}_{\mathcal{F}} e^{-\beta\left(\mathcal{H}_{L}^{(0)}-\mu \mathcal{N}_{L}\right)} \mathcal{V}_{L}\left(t_{1}\right) \cdots \mathcal{V}_{L}\left(t_{n}\right)}{\operatorname{Tr}_{\mathcal{F}} e^{-\beta\left(\mathcal{H}_{L}^{(0)}-\mu \mathcal{N}_{L}\right)}}
$$

where $\mathcal{V}_{L}(t)=e^{t\left(\mathcal{H}_{L}^{(0)}-\mu \mathcal{N}_{L}\right)} \mathcal{V}_{L} e^{-t\left(\mathcal{H}_{L}^{(0)}-\mu \mathcal{N}_{L}\right)}$ is the non-interacting $(U=0)$ version of the imaginary time evolution of $\mathcal{V}_{L}$, cf. with Eq.(3.1). Symmetrizing over the permutations of $t_{1}, \ldots, t_{n}$, this can be rewritten as

$$
\frac{\operatorname{Tr}_{\mathcal{F}} e^{-\beta\left(\mathcal{H}_{L}-\mu \mathcal{N}_{L}\right)}}{\operatorname{Tr}_{\mathcal{F}} e^{-\beta\left(\mathcal{H}_{L}^{(0)}-\mu \mathcal{N}_{L}\right)}}=1+\sum_{n \geq 1} \frac{(-U)^{n}}{n !} \int_{0}^{\beta} d t_{1} \cdots \int_{0}^{\beta} d t_{n}\left\langle\mathbf{T} \mathcal{V}_{L}\left(t_{1}\right) \cdots \mathcal{V}_{L}\left(t_{n}\right)\right\rangle_{\beta, L}^{(0)}
$$

where we recall that the label ${ }^{(0)}$ on the expectation symbol indicates that we are computing it at $U=0$. Since $\mathcal{H}_{L}^{(0)}-\mu \mathcal{N}_{L}$ is quadratic in the fermionic creation/annihilation operators, $\langle\cdot\rangle_{\beta, L}^{(0)}$ can be computed via the fermionic Wick rule, which is completely analogous to the one described for the infinite volume and zero temperature truncated expectation after (4.34), with the following minor differences: (i) since the expectation in (5.2) is not truncated, after having re-expressed $\mathcal{V}_{L}\left(t_{1}\right) \cdots \mathcal{V}_{L}\left(t_{n}\right)$ as a linear combination of monomials of order $4 n$ in the creation and annihilation operators, we have to sum over all possible pairings of these creation/annihilation operators, rather than just on the connected ones; (ii) the finite 
volume and finite temperature propagator associated with the pair $\left(\psi_{(t, \vec{x}), \sigma}^{-}, \psi_{\left(t^{\prime}, \vec{x}^{\prime}\right), \sigma^{\prime}}^{+}\right)$is

$$
\begin{aligned}
& g_{\sigma, \sigma^{\prime}}^{\beta, L}\left(t-t^{\prime}, \vec{x}-\vec{x}^{\prime}\right)=\left\langle\mathbf{T} \psi_{(t, \vec{x}), \sigma}^{-} \psi_{\left(t^{\prime}, \vec{x}^{\prime}\right), \sigma^{\prime}}^{+}\right\rangle_{\beta, L}^{(0)} \\
& =\frac{1}{L^{2}} \sum_{\vec{k} \in \mathcal{B}_{L}} e^{-i \vec{k}\left(\vec{x}-\vec{x}^{\prime}\right)}\left[e^{-\left(t-t^{\prime}\right)\left(\hat{H}^{(0)}(\vec{k})-\mu\right)}\left(\frac{\mathbb{1}\left(t>t^{\prime}\right)}{1+e^{-\beta\left(\hat{H}^{(0)}(\vec{k})-\mu\right)}}-\frac{\mathbb{1}\left(t \leq t^{\prime}\right) e^{-\beta\left(\hat{H}^{(0)}(\vec{k})-\mu\right)}}{1+e^{-\beta\left(\hat{H}^{(0)}(\vec{k})-\mu\right)}}\right)\right]_{\sigma, \sigma^{\prime}} .
\end{aligned}
$$

In the following, we denote by $g^{\beta, L}(t, \vec{x})$ the matrix whose elements are $g_{\sigma, \sigma^{\prime}}^{\beta, L}(t, \vec{x})$. Note that, if $0<t<\beta$, then $g^{\beta, L}(t-\beta, \vec{x})=-g^{\beta, L}(t, \vec{x})$. Therefore, it is natural to extend $g^{\beta, L}(t, \vec{x})$, which is a priori defined only on the time interval $(-\beta, \beta)$, to the whole real line, by anti-periodicity in the imaginary time, i.e., via the rule $g^{\beta, L}(t+n \beta, \vec{x})=(-1)^{n} g^{\beta, L}(t, \vec{x})$. The resulting extension can be expanded in Fourier series w.r.t. $t$, so that, for all $t \neq n \beta$,

$$
g^{\beta, L}(t, \vec{x})=\frac{1}{\beta L^{2}} \sum_{\substack{k_{0} \in \mathcal{B}_{\beta} \\ \vec{k} \in \mathcal{B}_{L}}} e^{-i \vec{k} \cdot \vec{x}-i k_{0} t} \hat{g}^{\beta, L}\left(k_{0}, \vec{k}\right)
$$

with $\mathcal{B}_{\beta}=\frac{2 \pi}{\beta}\left(\mathbb{Z}+\frac{1}{2}\right)$ and

$$
\hat{g}^{\beta, L}\left(k_{0}, \vec{k}\right):=\frac{1}{-i k_{0}+\hat{H}^{(0)}(\vec{k})-\mu} .
$$

If, instead, $t=n \beta$, then $g^{\beta, L}(n \beta, \vec{x})=(-1)^{n} \lim _{t \rightarrow 0^{-}} g^{\beta, L}(t, \vec{x})$. Note that, by the very definition of the propagator and the canonical anti-commutation relations, $g_{\sigma, \sigma^{\prime}}^{\beta, L}\left(0^{+}, \vec{x}\right)-$ $g_{\sigma, \sigma^{\prime}}^{\beta, L}\left(0^{-}, \vec{x}\right)=\delta_{\vec{x}, \overrightarrow{0}} \delta_{\sigma, \sigma^{\prime}}$, so that the only discontinuity points of $g^{\beta, L}(t, \vec{x})$ are $(n \beta, \overrightarrow{0})$.

In the following we will also need a variant of $g^{\beta, L}(t, \vec{x})$, to be denoted by $\bar{g}^{\beta, L}(t, \vec{x})$, which coincides with $g^{\beta, L}(t, \vec{x}), \forall(t, \vec{x}) \neq(n \beta, \overrightarrow{0})$, and with the arithmetic mean of $g^{\beta, L}\left(0^{+}, \overrightarrow{0}\right)$ and $g^{\beta, L}\left(0^{-}, \overrightarrow{0}\right)$ at the discontinuity points:

$$
\left.\bar{g}^{\beta, L}(\mathbf{x})\right|_{\mathbf{x}=(n \beta, \vec{x})}=\frac{g^{\beta, L}\left(0^{+}, \overrightarrow{0}\right)+g^{\beta, L}\left(0^{-}, \overrightarrow{0}\right)}{2} .
$$

The function $\bar{g}^{\beta, L}(\mathbf{x})$ is a natural object to introduce, in that it is the limit as $M \rightarrow \infty$ of a regularization of $g^{\beta, L}(\mathbf{x})$ obtained by cutting off the ultraviolet modes $\left|k_{0}\right|>2^{M}$ in the right side of (5.4). More specifically, if we take a smooth even compact support function $\chi_{0}(t)$, equal to 1 for $|t|<1$ and equal to 0 for $|t|>2$, and we define

$$
\bar{g}^{\beta, L, M}(\mathbf{x})=\frac{1}{\beta L^{2}} \sum_{\mathbf{k} \in \mathcal{B}_{\beta} \times \mathcal{B}_{L}} e^{-i \mathbf{k} \cdot \mathbf{x}} \chi_{0}\left(2^{-M} k_{0} / \delta_{\mu}\right) \hat{g}^{\beta, L}(\mathbf{k}),
$$

then

$$
\bar{g}^{\beta, L}(\mathbf{x})=\lim _{M \rightarrow \infty} \bar{g}^{\beta, L, M}(\mathbf{x}) .
$$

These propagators can be used to re-express the formal perturbation theory in (5.2) in terms of the limit of a regularized theory with finitely many degrees of freedom, which 
is advantageous for performing rigorous bounds on the convergence of the series. More precisely, we note that (5.2), as an identity between (a priori formal) power series, can be equivalently rewritten as

$$
\frac{\operatorname{Tr}_{\mathcal{F}} e^{-\beta\left(\mathcal{H}_{L}-\mu \mathcal{N}_{L}\right)}}{\operatorname{Tr}_{\mathcal{F}} e^{-\beta\left(\mathcal{H}_{L}^{(0)}-\mu \mathcal{N}_{L}\right)}}=\lim _{M \rightarrow \infty}\left[1+\sum_{n \geq 1} \frac{(-U)^{n}}{n !} \int_{0}^{\beta} d t_{1} \cdots \int_{0}^{\beta} d t_{n} \overline{\mathbb{E}}_{\beta, L, M}\left(\overline{\mathcal{V}}_{L}\left(t_{1}\right) \cdots \overline{\mathcal{V}}_{L}\left(t_{n}\right)\right)\right]
$$

where

$$
\overline{\mathcal{V}}_{L}(t)=\sum_{\vec{x}, \vec{y} \in \Lambda_{L}} \sum_{\sigma, \sigma^{\prime} \in I}\left(\psi_{(t, \vec{x}), \sigma}^{+} \psi_{(t, \vec{x}), \sigma}^{-}+\frac{1}{2}\right) v_{\sigma \sigma^{\prime}}(\vec{x}-\vec{y})\left(\psi_{(t, \vec{y}), \sigma^{\prime}}^{+} \psi_{(t, \vec{y}), \sigma^{\prime}}^{-}+\frac{1}{2}\right)
$$

and $\overline{\mathbb{E}}_{\beta, L, M}(\cdot)$ acts linearly on normal-ordered polynomials in $\psi_{(t, \vec{x}), \sigma}^{ \pm}$, the action on a normalordered monomial being defined by the fermionic Wick rule with propagator

$$
\overline{\mathbb{E}}_{\beta, L, M}\left(\psi_{(t, \vec{x}), \sigma}^{-} \psi_{\left(t^{\prime}, \vec{x}^{\prime}\right), \sigma^{\prime}}^{+}\right)=\bar{g}_{\sigma, \sigma^{\prime}}^{\beta, L, M}\left(t-t^{\prime}, \vec{x}-\vec{x}^{\prime}\right) .
$$

In order to check that the right side of (5.9) coincides order by order with the right side of (5.2), it is enough to note the following (assume, again without loss of generality, that the times $t_{1}, \ldots, t_{n}$ are all distinct):

- all the pairings contributing to $\left\langle\mathbf{T} \mathcal{V}_{L}\left(t_{1}\right) \cdots \mathcal{V}_{L}\left(t_{n}\right)\right\rangle_{\beta, L}^{(0)}$ without tadpoles (i.e., without contractions of two fields at the same space-time point) give the same contribution as the corresponding pairing in $\lim _{M \rightarrow \infty} \overline{\mathbb{E}}_{\beta, L, M}\left(\overline{\mathcal{V}}_{L}\left(t_{1}\right) \cdots \overline{\mathcal{V}}_{L}\left(t_{n}\right)\right)$, simply because $g^{\beta, L}(\mathbf{x})=\bar{g}^{\beta, L}(\mathbf{x}), \forall \mathbf{x} \neq(\beta n, \overrightarrow{0}) ;$

- in the pairings contributing to $\left\langle\mathbf{T} \mathcal{V}_{L}\left(t_{1}\right) \cdots \mathcal{V}_{L}\left(t_{n}\right)\right\rangle_{\beta, L}^{(0)}$ that contain tadpoles, every tadpole corresponds to a factor $\left\langle\psi_{(t, \vec{x}), \sigma}^{+} \psi_{(t, \vec{x}), \sigma}^{-}\right\rangle_{\beta, L}^{0}=-g_{\sigma, \sigma}^{\beta, L}\left(0^{-}, \overrightarrow{0}\right)$, while the corresponding tadpole in $\lim _{M \rightarrow \infty} \overline{\mathbb{E}}_{\beta, L, M}\left(\overline{\mathcal{V}}_{L}\left(t_{1}\right) \cdots \overline{\mathcal{V}}_{L}\left(t_{n}\right)\right)$ contributes a factor

$$
\left.\lim _{M \rightarrow \infty} \overline{\mathbb{E}}_{\beta, L, M}\left(\psi_{(t, \vec{x}), \sigma}^{+} \psi_{(t, \vec{x}), \sigma}^{-}\right)=-\bar{g}_{\sigma, \sigma}^{\beta, L}(0, \overrightarrow{0})=-\frac{1}{2}\left[g_{\sigma, \sigma}^{\beta, L}\left(0^{+}, \overrightarrow{0}\right)\right)+g_{\sigma, \sigma}^{\beta, L}\left(0^{-}, \overrightarrow{0}\right)\right] .
$$

The difference between the two is

$$
\left.-\bar{g}_{\sigma, \sigma}^{\beta, L}(0, \overrightarrow{0})+g_{\sigma, \sigma}^{\beta, L}(0, \overrightarrow{0})=-\frac{1}{2}\left[g_{\sigma, \sigma}^{\beta, L}\left(0^{+}, \overrightarrow{0}\right)\right)-g_{\sigma, \sigma}^{\beta, L}\left(0^{-}, \overrightarrow{0}\right)\right]=-\frac{1}{2},
$$

which is compensated exactly by the $+\frac{1}{2}$ 's appearing in the definition (5.10).

A concise way of rewriting the series in brackets in (5.9) is in terms of Grassmann integrals:

$$
1+\sum_{n \geq 1} \frac{(-U)^{n}}{n !} \int_{0}^{\beta} d t_{1} \cdots \int_{0}^{\beta} d t_{n} \overline{\mathbb{E}}_{\beta, L, M}\left(\overline{\mathcal{V}}_{L}\left(t_{1}\right) \cdots \overline{\mathcal{V}}_{L}\left(t_{n}\right)\right)=\int P_{\leq M}(d \Psi) e^{-U V_{\beta, L}(\Psi)},
$$

where $V_{\beta, L}(\Psi)$ and $\int P_{\leq M}(d \Psi)$ are, respectively, an element of a finite Grassmann algebra, and a linear map from the even part of the same algebra to the real numbers, defined as 
follows. Let $\mathcal{B}_{\beta}^{*}=\mathcal{B}_{\beta} \cap\left\{k_{0}: \chi_{0}\left(2^{-M} k_{0}\right)>0\right\}$, with $\mathcal{B}_{\beta}$ defined after (5.4), and $\mathcal{B}_{\beta, L}^{*}=$ $\mathcal{B}_{\beta}^{*} \times \mathcal{B}_{L}$. We consider the finite Grassmann algebra generated by the Grassmann variables $\left\{\hat{\Psi}_{\mathbf{k}, \sigma}^{ \pm}\right\}_{\mathbf{k} \in \mathcal{B}_{\beta, L}^{*}}^{\sigma \in I}$ and we let

$$
V_{L, \beta}(\Psi)=\sum_{\substack{\vec{x}, \vec{y} \in \Lambda_{L} \\ \sigma, \sigma^{\prime} \in I}} \int_{0}^{\beta} d t\left(\Psi_{(t, \vec{x}), \sigma}^{+} \Psi_{(t, \vec{x}), \sigma}^{-}+\frac{1}{2}\right) v_{\sigma \sigma^{\prime}}(\vec{x}-\vec{y})\left(\Psi_{(t, \vec{y}), \sigma^{\prime}}^{+} \Psi_{(t, \vec{y}), \sigma^{\prime}}^{-}+\frac{1}{2}\right)
$$

where

$$
\Psi_{\mathbf{x}, \sigma}^{ \pm}=\frac{1}{\beta L^{2}} \sum_{\mathbf{k} \in \mathcal{B}_{\beta, L}^{*}} e^{ \pm i \mathbf{k x}} \hat{\Psi}_{\mathbf{k}, \sigma}^{ \pm}
$$

Moreover, $\int P_{\leq M}(d \Psi)$ acts on a generic even monomial in the Grassmann variables as follows: it gives non zero only if the number of $\hat{\Psi}_{\mathbf{k}, \sigma}^{+}$variables is the same as the number of $\hat{\Psi}_{\mathbf{k}, \sigma}^{-}$ variables, in which case

$$
\int P_{\leq M}(d \Psi) \hat{\Psi}_{\mathbf{k}_{1}, \sigma_{1}}^{-} \hat{\Psi}_{\mathbf{p}_{1}, \sigma_{1}^{\prime}}^{+} \cdots \hat{\Psi}_{\mathbf{k}_{m}, \sigma_{m}}^{-} \hat{\Psi}_{\mathbf{p}_{m}, \sigma_{m}^{\prime}}^{+}=\operatorname{det}\left[C\left(\mathbf{k}_{i}, \sigma_{i} ; \mathbf{p}_{j}, \sigma_{j}^{\prime}\right)\right]_{i, j=1, \ldots, m}
$$

where $C\left(\mathbf{k}, \sigma ; \mathbf{p}, \sigma^{\prime}\right)=\beta L^{2} \delta_{\mathbf{k}, \mathbf{p}} \chi_{0}\left(2^{-M} k_{0} / \delta_{\mu}\right) \hat{g}_{\sigma, \sigma^{\prime}}^{\beta, L}(\mathbf{k})$. In particular,

$$
\int P_{\leq M}(d \Psi) \Psi_{\mathbf{x}}^{-} \Psi_{\mathbf{y}}^{+}=\bar{g}^{\beta, L, M}(\mathbf{x}-\mathbf{y})
$$

If needed, $\int P_{\leq M}(d \Psi)$ can be written explicitly in terms of the usual Berezin integral $\int d \Psi$, which is the linear functional on the Grassmann algebra acting non trivially on a monomial only if the monomial is of maximal degree, in which case

$$
\int d \Psi \prod_{\mathbf{k} \in \mathcal{B}_{\beta, L}^{*}} \prod_{\sigma \in I} \hat{\Psi}_{\mathbf{k}, \sigma}^{-} \hat{\Psi}_{\mathbf{k}, \sigma}^{+}=1
$$

The explicit expression of $\int P_{\leq M}(d \Psi)$ in terms of $\int d \Psi$ is

$$
\begin{aligned}
& \int P_{\leq M}(d \Psi)(\cdot)=\frac{1}{N_{\beta, L, M}} \int d \Psi \exp \left\{-\frac{1}{\beta L^{2}} \sum_{\mathbf{k} \in \mathcal{B}_{\beta, L}^{*}} \chi_{0}^{-1}\left(2^{-M} k_{0}\right) \hat{\Psi}_{\mathbf{k}, \cdot}^{+}\left[\hat{g}_{\mathbf{k}}^{\beta, L}\right]^{-1} \hat{\Psi}_{\mathbf{k}, \cdot}^{-}\right\}(\cdot), \\
& \text { with } \quad N_{\beta, L, M}=\prod_{\mathbf{k} \in \mathcal{B}_{\beta, L}^{*}}\left[\beta L^{2} \chi_{0}\left(2^{-M} k_{0} / \delta_{\mu}\right)\right]^{|I|} \operatorname{det} \hat{g}_{\mathbf{k}}^{\beta, L}
\end{aligned}
$$

which motivates the appellation "Gaussian integration" that is usually given to the reference "measure" $P_{\leq M}(d \Psi)$. Because of (5.15), $P_{\leq M}(d \Psi)$ is also called the Gaussian integration with propagator $\bar{g}^{\beta, L, M}$.

It is straightforward to check that the definitions above are given in such a way that the two sides of (5.11) coincide, order by order in $U$. Note, by the way, that (5.11) is a (finite) polynomial in $U$, for every finite $\beta, L, M$, simply because the Grassmann algebra entering the definition of the right side of (5.11) is finite. 
Summarizing,

$$
\frac{\operatorname{Tr}_{\mathcal{F}} e^{-\beta\left(\mathcal{H}_{L}-\mu \mathcal{N}_{L}\right)}}{\operatorname{Tr}_{\mathcal{F}} e^{-\beta\left(\mathcal{H}_{L}^{(0)}-\mu \mathcal{N}_{L}\right)}}=\lim _{M \rightarrow \infty} \int P_{\leq M}(d \Psi) e^{-U V_{\beta, L}(\Psi)},
$$

as an identity between (a priori formal) power series in $U$. In a similar way, one can show (details left to the reader) that the power series expansion for the truncated multipoint bond current-density correlations can be rewritten as

$$
\begin{aligned}
& \left\langle\mathbf{T}\left(J_{\vec{x}_{1} \vec{y}_{1}}^{\sigma_{1} \sigma_{1}^{\prime}}\right)_{t_{1}} ; \cdots ;\left(J_{\vec{x}_{m} \vec{y}_{m}}^{\sigma_{m} \sigma_{m}^{\prime}}\right)_{t_{m}} ; n_{\mathbf{x}_{m+1}}^{\sigma_{m+1}} ; \cdots ; n_{\mathbf{x}_{m+n}}^{\sigma_{m+n}}\right\rangle_{\beta, L}= \\
& \quad=\left.\lim _{M \rightarrow \infty} \frac{\partial^{m+n}}{\partial A_{\mathbf{x}_{1}, \vec{y}_{1}}^{\sigma_{1} \sigma_{1}^{\prime}} \cdots \partial \phi_{\mathbf{x}_{m+n}}^{\sigma_{m+n}}} \log \int P_{\leq M}(d \Psi) e^{-U V_{\beta, L}(\Psi)+(\phi, n)+(A, J)}\right|_{A=\phi=0},
\end{aligned}
$$

where

$$
\begin{aligned}
& (\phi, n)=\int_{0}^{\beta} d t \sum_{\vec{x}, \sigma} \phi_{(t, \vec{x})}^{\sigma}\left(\Psi_{(t, \vec{x}), \sigma}^{+} \Psi_{(t, \vec{x}), \sigma}^{-}+\frac{1}{2}\right) \\
& (A, J)=\int_{0}^{\beta} d t \sum_{\vec{x}, \vec{y}} \sum_{\sigma, \sigma^{\prime}} A_{(t, \vec{x}), \vec{y}}^{\sigma \sigma^{\prime}}\left[i \Psi_{(t, \vec{x}), \sigma}^{+} H_{\sigma \sigma^{\prime}}^{(0)}(\vec{x}-\vec{y}) \Psi_{(t, \vec{y}), \sigma^{\prime}}^{-}-i \Psi_{(t, \vec{y}), \sigma^{\prime}}^{+} H_{\sigma^{\prime} \sigma}^{(0)}(\vec{y}-\vec{x}) \Psi_{(t, \vec{x}), \sigma}^{-}\right] .
\end{aligned}
$$

The goal of the incoming discussion is to show that (5.17) and (5.18) are not just identities between formal power series, but rather between analytic functions of $U$. Recalling the connection between the total current and the bond current, (3.9), it is clear that this will in turn implies the same for the multipoint (total) current-density correlations. Therefore, from now on, we shall restrict our attention to the bond (rather than total) current-density correlations.

In order to prove that that (5.17) and (5.18) are identities between analytic functions, it actually suffices to prove the uniform analyticity in $M$, as $M \rightarrow \infty$, and the existence of the limit as $M \rightarrow \infty$ of the regularized free energy per site and correlations, as the following elementary lemma shows.

Lemma 5.1 Assume that, for any finite $\beta$ and $L$, there exists $\varepsilon_{\beta, L}>0$ such that the regularized free energy per site

$$
f_{\beta, L, M}=-\frac{1}{\beta L^{2}} \log \int P_{\leq M}(d \Psi) e^{-U V_{\beta, L}(\Psi)}
$$

and the regularized truncated correlations

$$
\begin{aligned}
& K^{\beta, L, M}\left(\mathbf{x}_{1}, \vec{y}_{1}, \sigma_{1}, \sigma_{1}^{\prime} ; \ldots ; \mathbf{x}_{m+n}, \sigma_{m+n}\right)= \\
& \quad=\left.\frac{\partial^{m+n}}{\partial A_{\mathbf{x}_{1}, \vec{y}_{1}}^{\sigma_{1} \sigma_{1}^{\prime}} \cdots \partial \phi_{\mathbf{x}_{m+n}}^{\sigma_{m+n}}} \log \int P_{\leq M}(d \Psi) e^{-U V_{\beta, L}(\Psi)+(\phi, n)+(A, J)}\right|_{A=\phi=0}
\end{aligned}
$$

are analytic functions of $U$ in the domain $D_{\beta, L}=\left\{U \in \mathbb{C}:|U|<\varepsilon_{\beta, L}\right\}$, uniformly in $M$ as $M \rightarrow \infty$. Moreover, assume that in any compact subset of $D_{\beta, L}$ the sequences $\left\{f_{\beta, L, M}\right\}_{M \geq 1}$ and $\left\{K^{\beta, L, M}\left(\mathbf{x}_{1}, \vec{y}_{1}, \sigma_{1}, \sigma_{1}^{\prime} ; \ldots ; \mathbf{x}_{m+n}, \sigma_{m+n}\right)\right\}_{M \geq 1}$ converge uniformly as $M \rightarrow$ $\infty$. Then (5.17) and (5.18) are valid as identities between analytic functions of $U$ in $D_{\beta, L}$. 
Remark 1 In the following we will prove the assumption of this lemma, and actually much more: namely, we will prove the analyticity of $f_{\beta, L, M}$ and $K^{\beta, L, M}\left(\mathbf{x}_{1}, \vec{y}_{1}, \sigma_{1}, \sigma_{1}^{\prime} ; \ldots ; \mathbf{x}_{m+n}, \sigma_{m+n}\right)$, uniformly in $\beta, L, M$ (not just in $M$ ). We will also prove that these functions converge not only as $M \rightarrow \infty$, but also as $L \rightarrow \infty$ and $\beta \rightarrow \infty$, which in turn implies that the limiting correlations in the thermodynamic and zero temperature limits are analytic as well, as claimed in Proposition 4.1.

Proof of Lemma 5.1. Let us start by proving (5.17), which is equivalent to

$$
\frac{\operatorname{Tr}_{\mathcal{F}} e^{-\beta\left(\mathcal{H}_{L}-\mu \mathcal{N}_{L}\right)}}{\operatorname{Tr}_{\mathcal{F}} e^{-\beta\left(\mathcal{H}_{L}^{(0)}-\mu \mathcal{N}_{L}\right)}}=\lim _{M \rightarrow \infty} e^{-\beta L^{2} f_{\beta, L, M}} .
$$

The first key remark is that, if $\beta, L$ are finite, the left side of this equation is an entire function of $U$, as it follows from the fact that the Fock space generated by the fermion operators $\psi_{\vec{x}, \sigma}^{ \pm}$, with $\vec{x} \in \Lambda_{L}, \sigma \in I$, is finite dimensional. On the other hand, by assumption, $f_{\beta, L, M}$ is analytic in $D_{\beta, L}$ and uniformly convergent as $M \rightarrow \infty$ in every compact subset of $D_{\beta, L}$. Hence, by Vitali's convergence theorem for analytic functions, the limit $f_{\beta, L}=$ $\lim _{M \rightarrow \infty} f_{\beta, L, M}$ is analytic in $D_{\beta, L}$ and its Taylor coefficients coincide with the limits as $M \rightarrow \infty$ of the Taylor coefficients of $f_{\beta, L, M}$. Moreover, by construction, as discussed after (5.9), the Taylor coefficients of $e^{-\beta L^{2} f_{\beta, L}}$ coincide with the Taylor coefficients of the left side of (5.22), which implies the validity of (5.22) as an identity between analytic functions in $D_{\beta, L}$, simply because the left side is entire in $U$, the right side is analytic in $D_{\beta, L}$ and the Taylor coefficients at the origin of the two sides are the same. By taking the logarithm at both sides, we also find that

$$
f_{\beta, L}=-\frac{1}{\beta L^{2}} \log \frac{\operatorname{Tr}_{\mathcal{F}} e^{-\beta\left(\mathcal{H}_{L}-\mu \mathcal{N}_{L}\right)}}{\operatorname{Tr}_{\mathcal{F}} e^{-\beta\left(\mathcal{H}_{L}^{(0)}-\mu \mathcal{N}_{L}\right)}}
$$

as an identity between analytic functions in $D_{\beta, L}$. In particular, the left side of (5.22) does not vanish on $D_{\beta, L}$.

In order to prove the analogous claim for the correlation functions, we note that the truncated correlations $\left\langle\mathbf{T}\left(J_{\vec{x}_{1} \vec{y}_{1}}^{\sigma_{1} \sigma_{t}^{\prime}}\right)_{t_{1}} ; \cdots ;\left(J_{\vec{x}_{m} \vec{y}_{m}}^{\sigma_{m}, \sigma_{m}^{\prime}}\right)_{t_{m}} ; n_{\mathbf{x}_{m+1}}^{\sigma_{m+1}} ; \cdots ; n_{\mathbf{x}_{m+n}}^{\sigma_{m+n}}\right\rangle_{\beta, L}$ are linear combination of ratios of entire functions, simply because they are linear combinations of products of non-truncated functions, each of which is a ratio of entire functions. The denominator in these ratios is proportional to a power of the left side of (5.22) that, as observed earlier, does not vanish on $D_{\beta, L}$. Therefore, the truncated correlations are analytic in $D_{\beta, L}$, which allow us to repeat the same argument used above for the free energy, to conclude the validity of (5.18) as well, as an identity between analytic functions in $D_{\beta, L}$.

\section{B. Uniform analyticity of the regularized correlation functions}

In this section, we prove the uniform analyticity of the regularized free energy per site and regularized correlations, in a domain $D$ independent not only of $M$, but also of $\beta, L$. 
Later, we will discuss the existence of the limit as $M, L, \beta \rightarrow \infty$ of the regularized functions, thus proving the assumptions of Lemma 5.1, as well as the existence and analyticity of the infinite volume and zero temperature limits. Throughout the proof, $C, C_{i}, c, c_{i}, \ldots$, stand for unspecified constants, independent of $\beta, L, M$ and of $\delta_{\mu}$, unless specified otherwise. The key result proved in this section is the following.

Lemma 5.2 There exists $\varepsilon_{0}=\varepsilon_{0}\left(\delta_{\mu}\right)>0$ such that the regularized free energy $f_{\beta, L, M}$ and correlations $K^{\beta, L, M}\left(\mathbf{x}_{1}, \vec{y}_{1}, \sigma_{1}, \sigma_{1}^{\prime} ; \ldots ; \mathbf{x}_{m+n}, \sigma_{m+n}\right)$ are analytic in the common analyticity domain $D_{0}=\left\{U:|U| \leq \varepsilon_{0}\right\}$. Moreover, the regularized correlations are translation invariant and they satisfy the cluster property with faster-than-any-power decay rate, i.e., for any collection of integers $\underline{m}=\left\{m_{i, j}, m_{k}\right\}_{k=1, \ldots, m}^{i, j=1, \ldots, m+n} \geq 0$, there exists a constant $C_{\underline{m}}=$ $C_{\underline{m}}\left(\delta_{\mu}\right)$ such that

$$
\frac{1}{\beta L^{2}} \int_{\Lambda_{\beta, L}^{m+n}} d \underline{\mathbf{x}} \sum_{\underline{\vec{y} \in \Lambda_{L}^{m}}}\left|K^{\beta, L, M}\left(\mathbf{x}_{1}, \vec{y}_{1}, \sigma_{1}, \sigma_{1}^{\prime} ; \ldots ; \mathbf{x}_{m+n}, \sigma_{m+n}\right)\right| d_{\underline{m}}(\underline{\mathbf{x}}, \underline{\vec{y}}) \leq C_{\underline{m}} .
$$

Here $\underline{\mathbf{x}}=\left\{\mathbf{x}_{1}, \ldots, \mathbf{x}_{m+n}\right\}, \underline{\vec{y}}=\left\{\vec{y}_{1}, \ldots, \vec{y}_{m}\right\}, \Lambda_{\beta, L}=(0, \beta) \times \Lambda_{L}, \int_{\Lambda_{\beta, L}} d \mathbf{x}$ is a shorthand for $\int_{0}^{\beta} d x_{0} \sum_{\vec{x} \in \Lambda_{L}}$, and $d_{\underline{m}, \underline{m}^{\prime}}(\underline{\mathbf{x}}, \underline{\vec{y}})=\prod_{i, j=1}^{m+n}\left|\mathbf{x}_{i}-\mathbf{x}_{j}\right|^{m_{i, j}} \prod_{k=1}^{m}\left|\vec{y}_{k}-\vec{x}_{k}\right|_{L}^{m_{k}}$, where, if $\left|x_{0}\right|_{\beta}=$ $\min _{n \mathbb{Z}}\left|x_{0}+n \beta\right|$ is the distance on the one-dimensional torus of size $\beta$ and $|\vec{x}|_{L}$ is the distance on the torus $\Lambda_{L}$, we denoted $|\mathbf{x}|=\mathfrak{e}_{0}\left|x_{0}\right|_{\beta}+|\vec{x}|_{L}$, with $\mathfrak{e}_{0}$ the energy scale defined in (2.10).

Proof of Lemma 5.2. The proof is long and, therefore, we split it into three main steps: we first define the multiscale decomposition of the Grassmann integral, which we intend to perform in an iterative fashion; next, we explain in detail how to integrate the first scale; finally, we explain the iterative procedure, whose output is conveniently organized in the form of a tree expansion.

Multiscale decomposition. In order to prove the analyticity of the regularized free energy and correlations, we perform the Grassmann integration in a multiscale fashion, by rewriting the propagator $\bar{g}^{\beta, L, M}$ as a sum of smooth "single scale" propagators $g^{(h)}, h=0,1, \ldots, M$, each decaying faster than any power on a specific time scale $\sim 2^{h}$ :

$$
\bar{g}^{\beta, L, M}(\mathbf{x})=\sum_{h=0}^{M} g^{(h)}(\mathbf{x}), \quad g^{(h)}(\mathbf{x})=\frac{1}{\beta L^{2}} \sum_{\mathbf{k} \in \mathcal{B}_{\beta, L}^{*}} e^{-i \mathbf{k} \cdot \mathbf{x}} \frac{f_{h}\left(k_{0}\right)}{-i k_{0}+\hat{H}^{(0)}(\vec{k})-\mu} .
$$

Here $f_{h}\left(k_{0}\right)=\chi_{0}\left(2^{-h} k_{0} / \delta_{\mu}\right)-\chi_{0}\left(2^{-h+1} k_{0} / \delta_{\mu}\right)$ for $h \geq 1$ and $f_{0}\left(k_{0}\right)=\chi_{0}\left(k_{0} / \delta_{\mu}\right)$. For later use, note that the single scale propagator $g^{(h)}(\mathbf{x})$ satisfies the bound

$$
\left|g^{(h)}(\mathbf{x})\right| \leq \frac{C_{K}}{1+\left(2^{h} \delta_{\mu}\left|x_{0}\right|_{\beta}+\left(\delta_{\mu} / \mathfrak{e}_{0}\right)|\vec{x}|_{L}\right)^{K}},
$$

for all $h, K$ such that $0 \leq h \leq M, K \geq 0$. In particular,

$$
\left\|g^{(h)}\right\|_{1, n}:=\int d \mathbf{x}\left\|g^{(h)}(\mathbf{x})\right\| \cdot|\mathbf{x}|^{n} \leq C_{n} \delta_{\mu}^{-3-n} 2^{-h} .
$$


where $\int d \mathbf{x} \equiv \int_{\Lambda_{\beta, L}} d \mathbf{x}$ is a shorthand for $\int_{0}^{\beta} d x_{0} \sum_{\vec{x} \in \Lambda_{L}}$. If $n=0$, we shall denote $\left\|g^{(h)}\right\|_{1}=$ $\left\|g^{(h)}\right\|_{1,0}$. Moreover, $g^{(h)}(\mathbf{x})$ admits a Gram decomposition, which will be useful in deriving combinatorially optimal bounds on the generic order of perturbation theory:

$$
g_{\sigma_{1}, \sigma_{2}}^{(h)}(\mathbf{x}-\mathbf{y})=\left(A_{h, \mathbf{x}, \sigma_{1}}, B_{h, \mathbf{y}, \sigma_{2}}\right) \equiv \sum_{\sigma^{\prime}} \int d \mathbf{z} A_{h, \mathbf{x}, \sigma_{1}}^{*}\left(\mathbf{z}, \sigma^{\prime}\right) \cdot B_{h, \mathbf{y}, \sigma_{2}}\left(\mathbf{z}, \sigma^{\prime}\right)
$$

with

$$
\begin{aligned}
& A_{h, \mathbf{x}, \sigma}\left(\mathbf{z}, \sigma^{\prime}\right)=\frac{1}{\beta L^{2}} \sum_{\mathbf{k} \in \mathcal{B}_{\beta, L}^{*}} e^{i \mathbf{k}(\mathbf{x}-\mathbf{z})} \sqrt{f_{h}\left(k_{0}\right)}\left[\frac{1}{k_{0}^{2}+\left(\hat{H}_{0}(\vec{k})-\mu\right)^{2}}\right]_{\sigma^{\prime} \sigma}, \\
& B_{h, \mathbf{x}, \sigma}\left(\mathbf{z}, \sigma^{\prime}\right)=\frac{1}{\beta L^{2}} \sum_{\mathbf{k} \in \mathcal{B}_{\beta, L}^{*}} e^{i \mathbf{k}(\mathbf{x}-\mathbf{z})} \sqrt{f_{h}\left(k_{0}\right)}\left[i k_{0}+\hat{H}_{0}(\vec{k})-\mu\right]_{\sigma^{\prime} \sigma}
\end{aligned}
$$

and

$$
\left\|A_{h, \mathbf{x}, \sigma}\right\|^{2}:=\left(A_{h, \mathbf{x}, \sigma}, A_{h, \mathbf{x}, \sigma}\right) \leq C\left(\delta_{\mu} 2^{h}\right)^{-3}, \quad\left\|B_{h, \mathbf{x}, \sigma}\right\|^{2} \leq C\left(\delta_{\mu} 2^{h}\right)^{3} .
$$

The decomposition (5.24) of the propagator allows us to compute the regularized Grassmann generating function,

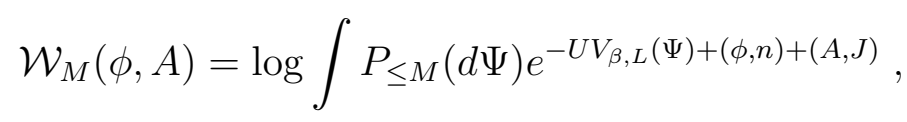

in an iterative way, by first integrating the degrees of freedom corresponding to $g^{(M)}$, then those corresponding to $g^{(M-1)}$, and so on. Technically, we make use of the so-called addition formula for Grassmann Gaussian integrations: if $g_{1}, g_{2}$ are two propagators and $g:=g_{1}+g_{2}$, then the Gaussian integration $P_{g}(d \psi)$ with propagator $g$ can be rewritten as $P_{g}(d \psi)=$ $P_{g_{1}}\left(d \psi_{1}\right) P_{g_{2}}\left(d \psi_{2}\right)$, in the sense that for every polynomial $f$

$$
\int P_{g}(d \psi) f(\psi)=\int P_{g_{1}}\left(d \psi_{1}\right) \int P_{g_{2}}\left(d \psi_{2}\right) f\left(\psi_{1}+\psi_{2}\right) \text {. }
$$

In our context, we rewrite $P_{\leq M}(d \Psi)=\prod_{h=0}^{M} P_{h}\left(d \Psi^{(h)}\right)$, where $P_{h}\left(d \Psi^{(h)}\right)$ is the Gaussian integration with propagator $g^{(h)}$, so that

$$
e^{\mathcal{W}_{M}(\phi, A)}=\int P_{0}\left(d \Psi^{(0)}\right) \cdots P_{h}\left(\Psi^{(h)}\right) e^{-\mathcal{V}^{(h)}\left(\Psi^{(\leq h)}, \phi, A\right)},
$$

where $\Psi^{(\leq h)}:=\sum_{j=0}^{h} \Psi^{(j)}$, so that

$$
\mathcal{V}^{(h)}(\Psi, \phi, A)=-\log \int P_{h+1}\left(d \Psi^{(h+1)}\right) \cdots P_{M}\left(\Psi^{(M)}\right) e^{-U V_{\beta, L}\left(\Psi+\Psi^{(h+1)}+\cdots+\Psi^{(M)}\right)+(\phi, n)+(A, J)} .
$$

and $\mathcal{V}^{(M)}(\Psi, \phi, A)=U V_{\beta, L}(\Psi)-(\phi, n)-(A, J)$. 
The first integration step. In order to compute the sequence $\mathcal{V}^{(h)}$ iteratively, let us start by explaining in detail the first step:

$$
\mathcal{V}^{(M-1)}(\Psi, \phi, A)=-\log \int P_{M}\left(d \Psi^{(M)}\right) e^{-\mathcal{V}^{(M)}\left(\Psi+\Psi^{(M)}, \phi, A\right)} .
$$

The logarithm in the right side can be expressed as a series of truncated expectations:

$$
\begin{aligned}
\log & \int P_{M}\left(d \Psi^{(M)}\right) e^{-\mathcal{V}^{(M)}\left(\Psi+\Psi^{(M)}, \phi, A\right)}= \\
= & \sum_{s \geq 1} \frac{(-1)^{s}}{s !} \mathcal{E}_{M}^{T}(\underbrace{\mathcal{V}^{(M)}\left(\Psi+\Psi^{(M)}, \phi, A\right) ; \cdots ; \mathcal{V}^{(M)}\left(\Psi+\Psi^{(M)}, \phi, A\right)}_{s \text { times }}),
\end{aligned}
$$

where

$$
\mathcal{E}_{M}^{T}\left(X_{1}\left(\Psi^{(M)}\right) ; \cdots ; X_{s}\left(\Psi^{(M)}\right)\right)=\left.\frac{\partial^{s}}{\partial \lambda_{1} \cdots \partial \lambda_{s}} \log \int P_{M}\left(d \Psi^{(M)}\right) e^{\lambda_{1} X_{1}\left(\Psi^{(M)}\right)+\cdots+\lambda_{s} X_{s}\left(\Psi^{(M)}\right)}\right|_{\lambda_{i}=0},
$$

and the $X_{i}$ 's are all even elements of the Grassmann algebra generated by the field $\Psi^{(M)}$ we are integrating over and by the "external" Grassmann field $\Psi$. The functional $\mathcal{E}_{M}^{T}$ is multilinear in its arguments, the action on a collection of monomials being defined by the truncated Wick rule with propagator $g^{(M)}$, which, as already explained above, is similar to the usual fermionic Wick rule, modulo the extra condition that, if the number $s$ of monomials involved is $\geq 2$, then the pairings one has to sum over are only those for which the collection of monomials $X_{1}, \ldots, X_{s}$ is connected (this means that for all $\mathcal{I} \subsetneq\{1, \ldots, s\}$, there exists at least one contracted pair involving one variable in the group $\left\{X_{i}\right\}_{i \in \mathcal{I}}$ and one in $\left\{X_{i}\right\}_{i \in \mathcal{I}^{c}}$ ).

A convenient representation of the truncated expectation, due to Battle, Brydges and Federbush [7, 21, 22], is the following (for a proof, see, e.g., [32, 33]). For a given (ordered) set of indices $P=\left(f_{1}, \ldots, f_{p}\right)$, with $f_{i}=\left(\mathbf{x}_{i}, \sigma_{i}, \varepsilon_{i}\right)$, let

$$
\Psi_{P}:=\Psi_{\mathbf{x}\left(f_{1}\right), \sigma\left(f_{1}\right)}^{\varepsilon\left(f_{1}\right)} \cdots \Psi_{\mathbf{x}\left(f_{p}\right), \sigma\left(f_{p}\right)}^{\varepsilon\left(f_{p}\right)},
$$

where $\mathbf{x}\left(f_{i}\right)=\mathbf{x}_{i}$, etc. It is customary to represent each variable $\Psi_{\mathbf{x}(f), \sigma(f)}^{\varepsilon(f)}$ as an oriented half-line, emerging from the point $\mathbf{x}(f)$ and carrying an arrow, pointing in the direction entering or exiting the point, depending on whether $\varepsilon(f)$ is equal to - or + , respectively; moreover, the half-line carries the labels $\sigma(f) \in I$. Given $n$ sets of indices $P_{1}, \ldots, P_{n}$, we can enclose the points $\mathbf{x}(f)$ belonging to the set $P_{j}$ in a box: in this way, assuming that all the points $\mathbf{x}(f), f \in \cup_{i} P_{i}$, are distinct, we obtain $n$ disjoint boxes. Given these definitions, if $\sum_{i=1}^{s}\left|P_{i}\right|$ is even we can write

$$
\mathcal{E}_{M}^{T}\left(\Psi_{P_{1}} ; \ldots ; \Psi_{P_{s}}\right)=\sum_{T \in \mathbf{T}_{M}} \alpha_{T} \prod_{\ell \in T} g_{\ell}^{(M)} \int d P_{T}(\mathbf{t}) \operatorname{det} G_{T}^{(M)}(\mathbf{t}),
$$

where: 
- any element $T$ of the set $\mathbf{T}_{M}=\mathbf{T}_{M}\left(P_{1}, \ldots, P_{s}\right)$ is a set of lines forming an anchored tree between the boxes $P_{1}, \ldots, P_{s}$, i.e., $T$ is a set of lines that becomes a tree if one identifies all the points in the same box; each line $\ell$ corresponds to a pair of halflines indexed by two distinct variables $f, f^{\prime} \in \cup_{i} P_{i}$ such that $\varepsilon(f)=-\varepsilon\left(f^{\prime}\right)$ (i.e., the directions of the two half-lines have to be compatible); if $\ell$ is obtained by contracting $f$ and $f^{\prime}$, we shall write $\ell=\left(f, f^{\prime}\right)$, with the convention that $\varepsilon\left(f^{\prime}\right)=-\varepsilon(f)=+$.

- $\alpha_{T}$ is a sign (irrelevant for the subsequent bounds), which depends on the choice of the anchored tree $T$;

- if $\ell=\left(f, f^{\prime}\right)$, then $g_{\ell}^{(M)}$ stands for $g_{\sigma(f), \sigma\left(f^{\prime}\right)}^{(M)}\left(\mathbf{x}(f)-\mathbf{x}\left(f^{\prime}\right)\right)$;

- if $\mathbf{t}=\left\{t_{i, i^{\prime}} \in[0,1], 1 \leq i, i^{\prime} \leq n\right\}$, then $d P_{T}(\mathbf{t})$ is a probability measure (depending on the anchored tree $T$ ) with support on a set of $\mathbf{t}$ such that $t_{i, i^{\prime}}=\mathbf{u}_{i} \cdot \mathbf{u}_{i^{\prime}}$ for some family of vectors $\mathbf{u}_{i} \in \mathbb{R}^{s}$ of unit norm;

- if $2 N=\sum_{i=1}^{s}\left|P_{i}\right|$, then $G_{T}^{(M)}(\mathbf{t})$ is a $(N-s+1) \times(N-s+1)$ matrix (depending both on the sets $P_{i}$ and on the anchored tree $\left.T\right)$, whose elements are given by $\left[G_{T}^{(M)}(\mathbf{t})\right]_{f, f^{\prime}}=$ $t_{i(f), i\left(f^{\prime}\right)} g_{\left(f, f^{\prime}\right)}^{(M)}$, where $f, f^{\prime} \in \cup_{i} P_{i} \backslash \cup_{\ell \in T}\left\{f_{\ell}^{-}, f_{\ell}^{+}\right\} \quad\left(\right.$ with $\left.\ell=\left(f_{\ell}^{-}, f_{\ell}^{+}\right)\right)$, and $i(f) \in$ $\{1, \ldots, s\}$ is the index such that $f \in P_{i(f)}$.

If $s=1$ the sum over $T$ is empty, but we can still use the Eq.(5.38) by interpreting the r.h.s. as equal to 1 if $P_{1}$ is empty and equal to $\operatorname{det} G^{T}(\mathbf{1})$ otherwise.

In order to use (5.38) in (5.33)-(5.34), we first rewrite $\mathcal{V}^{(M)}$ as

$$
\mathcal{V}^{(M)}(\Psi, \phi, A)=E_{M}(\phi)+\sum_{\rho=1}^{4} \sum_{\sigma, \sigma^{\prime} \in I} \int d \mathbf{x} d \mathbf{y} K_{\sigma \sigma^{\prime}}^{\rho}(\mathbf{x}, \mathbf{y})\left[\phi_{\mathbf{x}}^{\sigma}\right]^{\delta_{\rho, 1}}\left[A_{\mathbf{x}, \mathbf{y}}^{\sigma \sigma^{\prime}}\right]^{\delta_{\rho, 2}} \Psi_{P^{\rho}},
$$

where $E_{M}(\phi)=\frac{\beta L^{2}}{4} U \sum_{\sigma} \nu_{\sigma}-\frac{1}{2} \sum_{\sigma} \int d \mathbf{x} \phi_{\mathbf{x}}^{\sigma}$, with $\nu_{\sigma}=\sum_{\vec{x} \in \Lambda_{L}} \sum_{\sigma^{\prime} \in I} v_{\sigma \sigma^{\prime}}(\vec{x})$. Moreover, $A_{\mathbf{x}, \mathbf{y}}^{\sigma \sigma^{\prime}}=A_{\mathbf{x}, \vec{y}}^{\sigma \sigma^{\prime}}-A_{\mathbf{y}, \vec{x}}^{\sigma^{\prime} \sigma}$,

$$
\begin{aligned}
& K_{\sigma \sigma^{\prime}}^{1}(\mathbf{x}, \mathbf{y})=-\delta_{\sigma, \sigma^{\prime}} \delta(\mathbf{x}-\mathbf{y}), \quad K_{\sigma \sigma^{\prime}}^{2}(\mathbf{x}, \mathbf{y})=-i \delta\left(x_{0}-y_{0}\right) H_{\sigma \sigma^{\prime}}^{(0)}(\vec{x}-\vec{y}) \\
& K_{\sigma \sigma^{\prime}}^{3}(\mathbf{x}, \mathbf{y})=U \nu_{\sigma} \delta_{\sigma, \sigma^{\prime}} \delta(\mathbf{x}-\mathbf{y}), \quad K_{\sigma \sigma^{\prime}}^{4}(\mathbf{x}, \mathbf{y})=U \delta\left(x_{0}-y_{0}\right) v_{\sigma \sigma^{\prime}}(\vec{x}-\vec{y}),
\end{aligned}
$$

and

$$
\begin{aligned}
& P^{1}=P^{2}=P^{3}=\left((\mathbf{x}, \sigma,+),\left(\mathbf{y}, \sigma^{\prime},-\right)\right), \\
& P^{4}=\left((\mathbf{x}, \sigma,+),(\mathbf{x}, \sigma,-),\left(\mathbf{y}, \sigma^{\prime},+\right),\left(\mathbf{y}, \sigma^{\prime},-\right)\right) .
\end{aligned}
$$

Plugging (5.39) into (5.33)-(5.34), we obtain

$$
\begin{aligned}
& \mathcal{V}^{(M-1)}(\Psi, \phi, A)=E_{M}(\phi)-\sum_{s \geq 1} \frac{(-1)^{s}}{s !} \sum_{\substack{\rho_{1}, \ldots, \rho_{s} \\
\sigma_{1}, \sigma_{1}^{\prime}, \ldots, \sigma_{s}, \sigma_{s}^{\prime}}} \int \mathbf{x}_{1} d \mathbf{y}_{1} \cdots d \mathbf{x}_{s} d \mathbf{y}_{s} \times \\
& \times\left[\prod_{i: \rho_{i}=1} \phi_{\mathbf{x}_{i}}^{\sigma_{i}}\right]\left[\prod_{i: \rho_{i}=2} A_{\mathbf{x}_{i}, \mathbf{y}_{i}}^{\sigma_{i} \sigma_{i}^{\prime}}\right]\left[\prod_{i=1}^{s} K_{\sigma_{i} \sigma_{i}^{\prime}}^{\rho_{i}}\left(\mathbf{x}_{i}, \mathbf{y}_{i}\right)\right] \mathcal{E}_{M}^{T}\left(\left(\Psi+\Psi^{(M)}\right)_{\left.P_{1}^{\rho_{1}} ; \cdots ;\left(\Psi+\Psi^{(M)}\right)_{P_{s} \rho_{s}}\right) .}\right.
\end{aligned}
$$


The truncated expectation in the right side can be further rewritten as

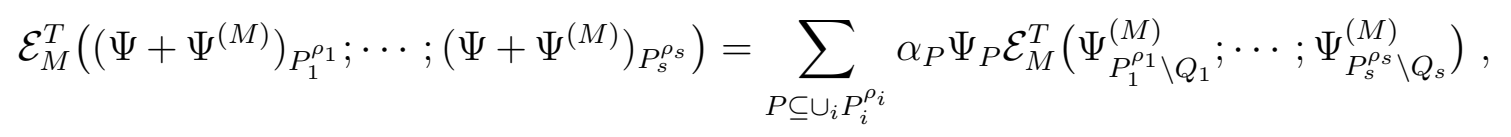

where $\alpha_{P}$ is a sign, and $Q_{i}=P \cap P_{i}^{\rho_{i}}$, so that, applying (5.38), we find

$$
\begin{gathered}
\mathcal{V}^{(M-1)}(\Psi, \phi, A)=E_{M}(\phi)-\sum_{s \geq 1} \frac{(-1)^{s}}{s !} \sum_{\underline{\rho}, \underline{\sigma}} \int d \underline{\mathbf{x}} d \underline{\mathbf{y}}\left[\prod_{i: \rho_{i}=1} \phi_{\mathbf{x}_{i}}^{\sigma_{i}}\right]\left[\prod_{i: \rho_{i}=2} A_{\mathbf{x}_{i}, \mathbf{y}_{i}}^{\sigma_{i}^{\prime}}\right] \times \\
\times\left[\prod_{i=1}^{s} K_{\sigma_{i} \sigma_{i}^{\prime}}^{\rho_{i}}\left(\mathbf{x}_{i}, \mathbf{y}_{i}\right)\right] \sum_{P \subseteq \cup_{i} P_{i}^{\rho_{i}}} \Psi_{P} \sum_{T \in \mathbf{T}_{M}} \alpha_{P, T} \prod_{\ell \in T} g_{\ell}^{(M)} \int d P_{T}(\mathbf{t}) \operatorname{det} G_{T}^{(M)}(\mathbf{t}),
\end{gathered}
$$

where $\underline{\rho}, \underline{\sigma}, \underline{\mathbf{x}}$ and $\underline{\mathbf{y}}$ are shorthands for $\left(\rho_{1}, \ldots, \rho_{s}\right),\left(\sigma_{1}, \sigma_{1}^{\prime}, \ldots, \sigma_{s}, \sigma_{s}^{\prime}\right),\left(\mathbf{x}_{1}, \ldots, \mathbf{x}_{s}\right)$ and $\left(\mathbf{y}_{1}, \ldots, \mathbf{y}_{s}\right)$, respectively, and $\alpha_{P, T}=\alpha_{P} \alpha_{T}$. Eq.(5.45) can be equivalently rewritten as

$$
\begin{aligned}
& \mathcal{V}^{(M-1)}(\Psi, \phi, A)=E_{M}(\phi)+ \\
& \left.+\sum_{n \geq 0} \sum_{s_{1}, s_{2} \geq 0} \sum_{\underline{\sigma}, \underline{\underline{\varepsilon}}} \int d \underline{\mathbf{x}} d \underline{\mathbf{y}} d \underline{\mathbf{z}} W_{2 n, s_{1}, s_{2}, \underline{\underline{\sigma}}, \underline{\underline{\mathbf{x}}}}^{(M-1)}, \underline{\mathbf{y}}, \underline{\mathbf{z}}\right)\left[\prod_{i=1}^{s_{1}} \phi_{\mathbf{x}_{i}}^{\sigma_{i}}\right]\left[\prod_{i=s_{1}+1}^{s_{1}+s_{2}} A_{\mathbf{x}_{i}, \mathbf{y}_{i}}^{\sigma_{i} \sigma_{i}^{\prime}}\right]\left[\prod_{i=1}^{2 n} \Psi_{\mathbf{z}_{i}, \sigma_{i}^{\prime \prime}}^{\varepsilon_{i}}\right]
\end{aligned}
$$

with

$$
\begin{aligned}
& W_{2 n, s_{1}, s_{2}, \underline{\underline{\sigma}}, \underline{\varepsilon}}^{(M-1)}(\underline{\mathbf{x}}, \underline{\mathbf{y}}, \underline{\mathbf{z}})=\sum_{\substack{s_{3} \geq 0 \\
s_{4} \geq n-1}}^{*} \frac{(-1)^{s-1}}{s_{1} ! s_{2} ! s_{3} ! s_{4} !} \sum_{\substack{\sigma_{i}, \sigma_{i}^{\prime}: \\
i>s_{1}+s_{2}}} \int\left[\prod_{i>s_{1}+s_{2}} d \mathbf{x}_{i} d \mathbf{y}_{i}\right] \times \\
& \times\left[\prod_{i=1}^{s} K_{\sigma_{i} \sigma_{i}^{\prime}}^{\bar{\rho}_{i}}\left(\mathbf{x}_{i}, \mathbf{y}_{i}\right)\right] \sum_{\substack{P \subseteq \cup_{i} P_{i}^{\bar{i}_{i}}: \\
|P|=2 n}} \delta\left(P-P_{e x t}\right) \sum_{T \in \mathbf{T}_{M}} \alpha_{P, T} \prod_{\ell \in T} g_{\ell}^{(M)} \int d P_{T}(\mathbf{t}) \operatorname{det} G_{T}^{(M)}(\mathbf{t}),
\end{aligned}
$$

where $s=s_{1}+s_{2}+s_{3}+s_{4}$, the $*$ on the sum indicates the constraint that $s \geq 1$, and $\bar{\rho}_{i}$ is equal to 1 if $i \leq s_{1}$, is equal to 2 if $0<i-s_{1} \leq s_{2}$, is equal to 3 if $0<i-s_{1}-s_{2} \leq s_{3}$, and is equal to 4 otherwise. Moreover, $P_{e x t}=\left(\left(\mathbf{z}_{1}, \sigma_{1}^{\prime \prime}, \varepsilon_{1}\right), \ldots,\left(\mathbf{z}_{2 n}, \sigma_{2 n}^{\prime \prime}, \varepsilon_{2 n}\right)\right)$, and $\delta\left(P-P_{e x t}\right)$ is a shorthand for the product of delta functions $\prod_{f_{i} \in P} \delta\left(\mathbf{x}\left(f_{i}\right)-\mathbf{z}_{i}\right) \delta_{\sigma\left(f_{i}\right), \sigma_{i}^{\prime \prime}} \delta_{\varepsilon\left(f_{i}\right), \varepsilon_{i}}$, where the labeling $P=\left(f_{1}, \ldots, f_{2 n}\right)$ is understood. Note that, in the case that $n=s_{1}=s_{2}=0$, in the right side of (5.46) there are neither sums over $\underline{\sigma}, \underline{\varepsilon}$ nor integrals over $\underline{\mathbf{x}}, \underline{\mathbf{y}}, \underline{\mathbf{z}}$, and $W_{0,0,0}^{(M-1)}$ is a constant, given by (5.46), with the understanding that the meaningless factors or sums or integrals should be replaced by one.

We are finally in the position of proving the analyticity of the integral kernels of $\mathcal{V}^{(M-1)}$. By using (5.46) we obtain

$$
\begin{aligned}
\frac{1}{\beta L^{2}} \int d \mathbf{x} d \mathbf{y} d \mathbf{z}\left|W_{2 n, s_{1}, s_{2}, \underline{\underline{\sigma}, \underline{\varepsilon}}}^{(M-1)}(\underline{\mathbf{x}}, \underline{\mathbf{y}}, \underline{\mathbf{z}})\right| \leq \\
\quad \leq \sum_{\substack{s_{3} \geq 0 \\
s_{4} \geq n-1}}^{*} \frac{|I|^{2 s_{3}+2 s_{4}}}{s_{1} ! s_{2} ! s_{3} ! s_{4} !}\left[\prod_{j=1}^{4}\left\|K^{j}\right\|_{1}^{s_{j}}\right]\left(\begin{array}{c}
2 s+2 s_{4} \\
2 n
\end{array}\right)\left(C^{s} s !\right)\left\|g^{(M)}\right\|_{1} \cdot\left\|\operatorname{det} G_{T}^{(M)}\right\|_{\infty}
\end{aligned}
$$


where: $|I|^{2 s_{3}+2 s_{4}}$ bounds the number of terms in the sum over $\sigma_{i}, \sigma_{i}^{\prime} ;\left\|K^{j}\right\|_{1}=\sup _{\sigma, \sigma^{\prime}}$ $\int d \mathbf{x}\left|K_{\sigma \sigma^{\prime}}^{j}(\mathbf{x}, \mathbf{0})\right| ;\left(\begin{array}{c}2 s+2 s_{4} \\ 2 n\end{array}\right)$ bounds the number of terms in the sum over $P$; $\left(C^{s} s !\right)$ bounds the number of terms in the sum over $T$. Recalling (5.26) for $n=0$ and the definitions (5.40)-(5.41), from which $\left\|K^{j}\right\|_{1} \leq C|U|^{\delta_{j, 3}+\delta_{j, 4}}$, we find that (5.48) implies

$$
\frac{1}{\beta L^{2}} \int d \mathbf{x} d \mathbf{y} d \mathbf{z}\left|W_{2 n, s_{1}, s_{2}, \underline{\sigma}, \underline{\varepsilon}}^{(M-1)}(\underline{\mathbf{x}}, \underline{\mathbf{y}}, \underline{\mathbf{z}})\right| \leq \sum_{\substack{s_{3} \geq 0 \\ s_{4} \geq n-1}}^{*} C^{s}|U|^{s_{3}+s_{4}}\left(\delta_{\mu}^{-3} 2^{-M}\right)^{s-1}\left\|\operatorname{det} G_{T}^{(M)}\right\|_{\infty} .
$$

In order to bound $\operatorname{det} G_{T}^{(M)}$, we use the Gram-Hadamard inequality, stating that, if $M$ is a square matrix with elements $M_{i j}$ of the form $M_{i j}=\left(A_{i}, B_{j}\right)$, where $A_{i}, B_{j}$ are vectors in a Hilbert space with scalar product $(\cdot, \cdot)$, then

$$
|\operatorname{det} M| \leq \prod_{i}\left\|A_{i}\right\| \cdot\left\|B_{i}\right\| \text {. }
$$

where $\|\cdot\|$ is the norm induced by the scalar product. In our case, $\left[G_{T}^{(M)}(\mathbf{t})\right]_{f, f^{\prime}}=\mathbf{u}_{i(f)}$. $\mathbf{u}_{i\left(f^{\prime}\right)}\left(A_{M, \mathbf{x}(f), \sigma(f)}, B_{M, \mathbf{x}\left(f^{\prime}\right), \sigma\left(f^{\prime}\right)}\right)$, so that, using (5.28) and recalling that $G_{T}^{(M)}$ is a $\left(s_{4}-n+\right.$ 1) $\times\left(s_{4}-n+1\right)$ matrix,

$$
\left\|\operatorname{det} G_{T}^{(M)}\right\|_{\infty} \leq C^{s_{4}-n+1} .
$$

Plugging this last ingredient into (5.49), we finally obtain

$$
\begin{aligned}
& \frac{1}{\beta L^{2}} \int d \mathbf{x} d \mathbf{y} d \mathbf{z}\left|W_{2 n, s_{1}, s_{2}, \underline{\underline{\sigma}}, \underline{\underline{\varepsilon}}}^{(M-1)}(\underline{\mathbf{x}}, \underline{\mathbf{y}}, \underline{\mathbf{z}})\right| \leq \sum_{\substack{s_{3} \geq 0 \\
s_{4} \geq n-1}}^{*} C^{s}|U|^{s_{3}+s_{4}}\left(\delta_{\mu}^{-3} 2^{-M}\right)^{s-1} \\
& \leq C^{n}|U|^{[n-1]_{+}}\left(\delta_{\mu}^{-3} 2^{-M}\right)^{\left[s_{1}+s_{2}+n-2\right]_{+}} \text {, }
\end{aligned}
$$

where $[\cdot]_{+}=\max \{\cdot, 0\}$ denotes the positive part. Eq.(5.52) proves the analyticity of the kernels of $\mathcal{V}^{(M)}$ for $U$ small enough, uniformly in $M$ (but not in $\delta_{\mu}$, in general).

Moreover, the kernels $W_{2 n, s_{1}, s_{2}, \underline{\underline{\sigma}, \underline{\varepsilon}}}^{(M-1)}(\underline{\mathbf{x}}, \underline{\mathbf{y}}, \underline{\mathbf{z}})$ decay faster than any power, on scale $\delta_{\mu}^{-1}$, in the relative distances between the coordinates $\mathbf{x}_{i}, \mathbf{y}_{i}, \mathbf{z}_{i}$. In order to prove this, we multiply the argument of the integral in the left side of (5.48) by a product of factors of the form $\left|\mathbf{x}_{i}-\mathbf{x}_{j}\right|^{m_{i, j}}$, or $\left|\mathbf{x}_{i}-\mathbf{y}_{j}\right|^{m_{i, j}^{\prime}}$, etc. We denote by $m=\sum_{i, j}\left(m_{i, j}+m_{i, j}^{\prime}+\cdots\right)$ the sum of these exponents. Again, we use the representation (5.46), and we decompose each factor "along the anchored tree T", that is we bound it by using

$$
\left|\mathbf{x}_{i}-\mathbf{x}_{j}\right| \leq \sum_{\ell \in T}\left|\mathbf{x}\left(f_{\ell}^{-}\right)-\mathbf{x}\left(f_{\ell}^{+}\right)\right|+\sum_{i=1}^{s} d_{i}
$$

where $d_{i}=\max _{f, f^{\prime} \in P_{T, i}}\left|\mathbf{x}(f)-\mathbf{x}\left(f^{\prime}\right)\right|$ and $P_{T, i}=\cup_{\ell \in T}\left\{f_{\ell}^{-}, f_{\ell}^{+}\right\} \cap P_{i}^{\bar{\rho}_{i}}$. In this way, the right side of (5.48) is replaced by a sum of terms, each of which is obtained by replacing some of the factors $\left\|K^{j}\right\|_{1}$ and $\left\|g^{(M)}\right\|_{1}$ by $\left\|K^{j}\right\|_{1, n_{i}}=\sup _{\sigma, \sigma^{\prime}} \int d \mathbf{x}\left|K_{\sigma \sigma^{\prime}}^{j}(\mathbf{x}, \mathbf{0})\right||\mathbf{x}|^{n_{i}} \leq C_{n_{i}}$ and by $\left\|g^{(M)}\right\|_{1, n_{i}^{\prime}}$, respectively. Recall that, by (5.26), the dimensional estimate of $\left\|g^{(M)}\right\|_{1, n_{i}^{\prime}}$ 
differs from that of $\left\|g^{(M)}\right\|_{1}$ just by a factor $\delta_{\mu}^{-n_{i}^{\prime}}$. Moreover, the total sum of the exponents $n_{i}, n_{i}^{\prime}$, etc., equals the exponent $m$ introduced earlier. Therefore, the product of the extra factors $\delta_{\mu}^{-n_{i}^{\prime}}$ is smaller than $\delta_{\mu}^{-m}$. All in all, the dimensional estimate on the kernels $W_{2 n, s_{1}, s_{2}, \underline{\sigma}, \underline{\varepsilon}}^{(M-1)}(\underline{\mathbf{x}}, \underline{\mathbf{y}}, \underline{\mathbf{z}})$, multiplied by the extra factors $\left|\mathbf{x}_{i}-\mathbf{x}_{i}\right|^{m_{i, j}}$, etc, is the same as (5.52), up to an extra factor $C_{m} \delta_{\mu}^{-m}$, for all $m \geq 0$.

The iterative integration procedure and the tree expansion. We are now in the position of iterating the procedure used above for computing the integral over the scale $M$. By using (5.32) and the definition of truncated expectation $\mathcal{E}_{h}^{T}$ (which is the same as (5.36), with $M$ replaced by $h$ ), we obtain

$$
\begin{aligned}
\mathcal{V}^{(h-1)}(\Psi, \phi, A) & =-\log \int P_{h}\left(d \Psi^{(h)}\right) e^{-\mathcal{V}^{(h)}\left(\Psi+\Psi^{(h)}, \phi, A\right)}= \\
& =\sum_{s \geq 1} \frac{(-1)^{s}}{s !} \mathcal{E}_{h}^{T}(\underbrace{\mathcal{V}^{(h)}\left(\Psi+\Psi^{(h)}, \phi, A\right) ; \cdots ; \mathcal{V}^{(h)}\left(\Psi+\Psi^{(h)}, \phi, A\right)}_{s \text { times }}) .
\end{aligned}
$$

Eq.(5.54) can be graphically represented as in Fig.1. The tree in the left side, consisting

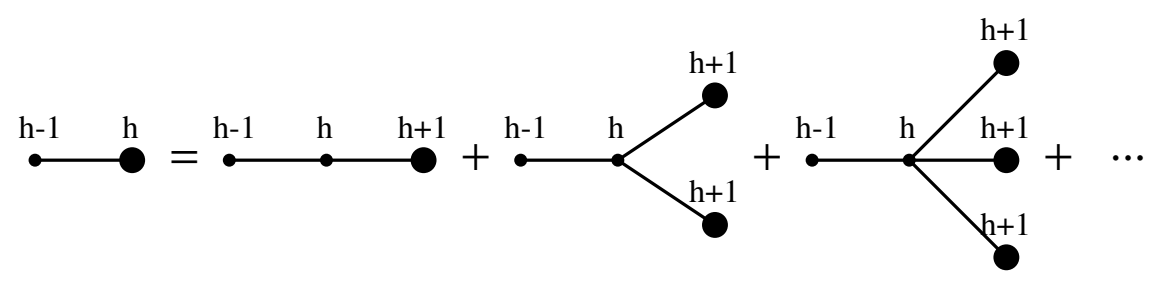

FIG. 1. The graphical representation of $\mathcal{V}^{(h-1)}$.

of a single horizontal branch, connecting the left node (called the root and associated with the scale label $h-1$ ) with a big black dot on scale $h$, represents $\mathcal{V}^{(h-1)}$. In the right side, the term with $s$ final points represents the corresponding term in the right side of (5.54): a scale label $h-1$ is attached to the leftmost node (the root); a scale label $h$ is attached to the central node (corresponding to the action of $\mathcal{E}_{h}^{T}$ ); a scale label $h+1$ is attached to the $s$ rightmost nodes with the big black dots (representing $\mathcal{V}^{(h)}$ ).

Iterating the graphical equation in Fig.1 up to scale $M$, and representing the endpoints on scale $M+1$ as simple dots (rather than big black dots), we end up with a graphical representation of $\mathcal{V}^{(h)}$ in terms of Gallavotti-Nicolò trees [30, 31], see Fig.2, defined in terms of the following features.

1. Let us consider the family of all trees which can be constructed by joining a point $r$, the root, with an ordered set of $N \geq 1$ points, the endpoints of the unlabeled tree, so that $r$ is not a branching point. $N$ will be called the order of the unlabeled tree and the branching points will be called the non trivial vertices. The unlabeled trees are partially ordered from the root to the endpoints in the natural way; we shall use the 


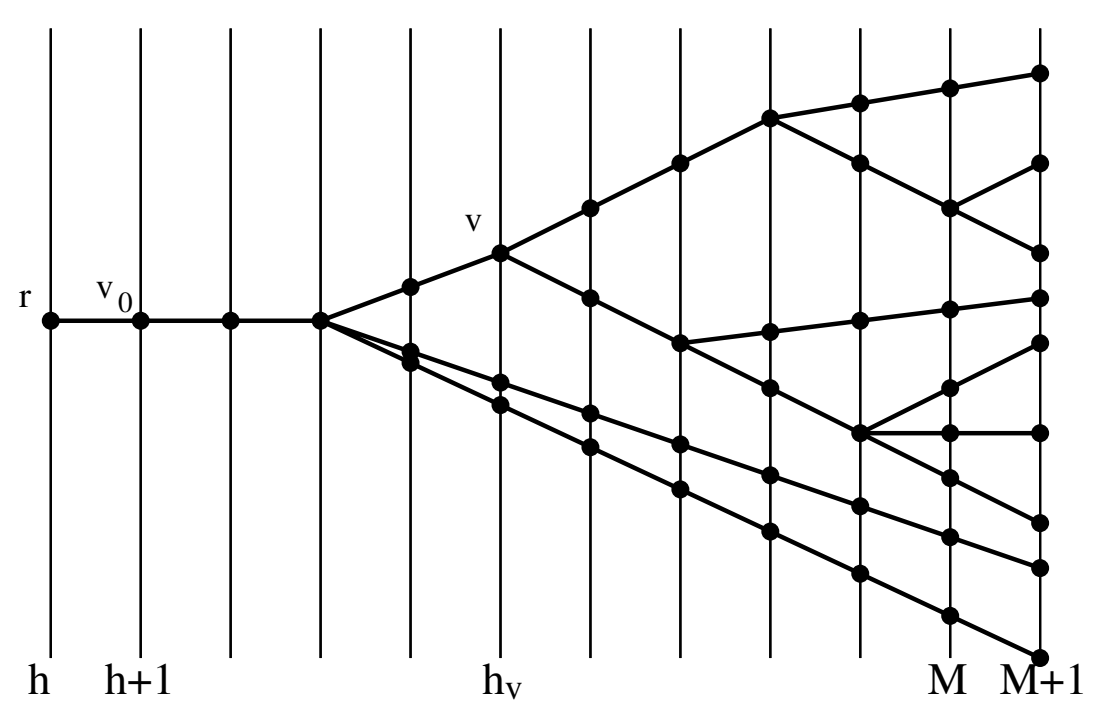

FIG. 2. A tree $\tau \in \widetilde{\mathcal{T}}_{M ; h, N}$ with $N=9$ : the root is on scale $h$ and the endpoints are on scale $M+1$.

symbol $<$ to denote the partial order. Two unlabeled trees are identified if they can be superposed by a suitable continuous deformation, so that the endpoints with the same index coincide. It is then easy to see that the number of unlabeled trees with $N$ end-points is bounded by $4^{N}$ (see, e.g., [32, appendix A.1.2] for a proof of this fact). We shall also consider the labeled trees (to be called simply trees in the following); they are defined by associating some labels with the unlabeled trees, as explained in the following items.

2. We associate a label $0 \leq h \leq M-1$ with the root and we denote by $\widetilde{\mathcal{T}}_{M ; h, N}$ the corresponding set of labeled trees with $N$ endpoints. Moreover, we introduce a family of vertical lines, labeled by an integer taking values in $[h, M+1]$, and we represent any tree $\tau \in \widetilde{\mathcal{T}}_{M ; h, N}$ so that, if $v$ is an endpoint, it is contained in the vertical line with index $h_{v}=M+1$, while if it is a non trivial vertex, it is contained in a vertical line with index $h<h_{v} \leq M$, to be called the scale of $v$; the root $r$ is on the line with index $h$. In general, the tree will intersect the vertical lines in set of points different from the root, the endpoints and the branching points; these points will be called trivial vertices. The set of the vertices will be the union of the endpoints, of the trivial vertices and of the non trivial vertices; note that the root is not a vertex. Every vertex $v$ of a tree will be associated to its scale label $h_{v}$, defined, as above, as the label of the vertical line whom $v$ belongs to. Note that, if $v_{1}$ and $v_{2}$ are two vertices and $v_{1}<v_{2}$, then $h_{v_{1}}<h_{v_{2}}$.

3. There is only one vertex immediately following the root, called $v_{0}$ and with scale label 
equal to $h+1$.

4. Given a vertex $v$ of $\tau \in \widetilde{\mathcal{T}}_{M ; h, N}$ that is not an endpoint, we can consider the subtrees of $\tau$ with root $v$, which correspond to the connected components of the restriction of $\tau$ to the vertices $w \geq v$. If a subtree with root $v$ contains only $v$ and one endpoint on scale $h_{v}+1$, it is called a trivial subtree.

5. With each endpoint $v$ we associate one of the terms contributing to $\mathcal{V}^{(M)}(\Psi, \phi, A)$, see (5.39). In order to distinguish between the various terms in the right side of (5.39), we introduce a type label $\rho_{v} \in\{0,1,2,3,4\}$. If $\rho_{v}=0$, then we associate the endpoint with a contribution $E_{M}(\phi)$, while, if $1 \leq \rho_{v} \leq 3$, then we associate the endpoint with a contribution $K_{\sigma_{v} \sigma_{v}^{\prime}}^{\rho_{v}}\left(\mathbf{x}_{v}, \mathbf{y}_{v}\right)\left[\phi_{\mathbf{x}_{v}}^{\sigma_{v}}\right]^{\delta_{\rho_{v}, 1}}\left[A_{\mathbf{x}_{v}, \mathbf{y}_{v}}^{\sigma_{v} \sigma_{v}^{\prime}}\right]^{\delta_{\rho_{v}, 2}} \Psi_{I_{v}}$.

The field labels attached to the endpoints $v$ of $\tau$ are denoted by $I_{v}$. If $\rho_{v}=0$, then $I_{v}=\emptyset$; if $\rho_{v}=1,2,3$, then $I_{v}=\left(\left(\mathbf{x}_{v}, \sigma_{v},+\right),\left(\mathbf{y}_{v}, \sigma_{v}^{\prime},-\right)\right)$; if $\rho_{v}=4$, then $I_{v}=$ $\left(\left(\mathbf{x}_{v}, \sigma_{v},+\right),\left(\mathbf{x}_{v}, \sigma_{v},-\right),\left(\mathbf{y}_{v}, \sigma_{v}^{\prime},+\right),\left(\mathbf{y}_{v}, \sigma_{v}^{\prime},-\right)\right)$. Moreover, given any vertex $v \in \tau$, we denote by $I_{v}$ the set of field labels associated with the endpoints following the vertex $v$; given $f \in I_{v}, \mathbf{x}(f), \sigma(f)$ and $\varepsilon(f)$ denote the space-time point, the $\sigma$ index and the $\varepsilon$ index of the Grassmann variable with label $f$. In the following, the "sum" over the field labels associated with the endpoints should be understood as $\sum_{\underline{\sigma}_{v_{0}}} \int d \underline{\mathbf{x}}_{v_{0}}$, where $v_{0}$ is the leftmost vertex of $\tau, \underline{\sigma}_{v}=\cup_{f \in I_{v}}\{\sigma(f)\}$ and $\underline{\mathbf{x}}_{v}=\cup_{f \in I_{v}}\{\mathbf{x}(f)\}$.

In terms of trees, the effective potential $\mathcal{V}^{(h)},-1 \leq h \leq M$ (with $\mathcal{V}^{(-1)}$ identified with $\left.\mathcal{W}_{M}\right)$, can be written as

$$
\mathcal{V}^{(h)}\left(\Psi^{(\leq h)}\right)=\sum_{N=1}^{\infty} \sum_{\tau \in \widetilde{\mathcal{T}}_{M ; h, N}} \widetilde{\mathcal{V}}^{(h)}\left(\tau, \Psi^{(\leq h)}\right),
$$

where, if $v_{0}$ is the first vertex of $\tau$ and $\tau_{1}, \ldots, \tau_{s}\left(s=s_{v_{0}}\right)$ are the subtrees of $\tau$ with root $v_{0}$, $\widetilde{\mathcal{V}}^{(h)}\left(\tau, \Psi^{(\leq h)}\right)$ is defined inductively as:

$$
\widetilde{\mathcal{V}}^{(h)}\left(\tau, \Psi^{(\leq h)}\right)=\frac{(-1)^{s-1}}{s !} \mathcal{E}_{h+1}^{T}\left[\widetilde{\mathcal{V}}^{(h+1)}\left(\tau_{1}, \Psi^{(\leq h+1)}\right) ; \ldots ; \widetilde{\mathcal{V}}^{(h+1)}\left(\tau_{s}, \Psi^{(\leq h+1)}\right)\right] .
$$

where, if $\tau$ is a trivial subtree with root on scale $M$, then $\widetilde{\mathcal{V}}^{(M)}\left(\tau, \Psi^{(\leq M)}\right)=\mathcal{V}^{(M)}\left(\Psi^{(\leq M)}\right)$ (for lightness of notation, we are dropping the arguments $(\phi, A)$, which are implicitly understood here and in the following).

For what follows, it is important to specify the action of the truncated expectations on the branches connecting any endpoint $v$ to the closest non-trivial vertex $v^{\prime}$ preceding it. In fact, if $\tau$ has only one end-point, it is convenient to rewrite $\widetilde{\mathcal{V}}^{(h)}\left(\tau, \Psi^{(\leq h)}\right)=$ $\mathcal{E}_{h+1}^{T} \mathcal{E}_{h+2}^{T} \cdots \mathcal{E}_{M}^{T}\left(\mathcal{V}\left(\Psi^{(\leq M)}\right)\right) \equiv \widetilde{\mathcal{V}}^{(h)}\left(\Psi^{(\leq h)}\right)$ as:

$$
\widetilde{\mathcal{V}}^{(h)}\left(\Psi^{(\leq h)}\right)=\mathcal{V}^{(M)}\left(\Psi^{(\leq h)}\right)+\mathcal{E}_{h+1}^{T} \cdots \mathcal{E}_{M}^{T}\left(\mathcal{V}^{(M)}\left(\Psi^{(\leq M)}\right)-\mathcal{V}^{(M)}\left(\Psi^{(\leq h)}\right)\right) .
$$


The second term in the right side can be evaluated explicitly and gives:

$$
\mathcal{E}_{h+1}^{T} \cdots \mathcal{E}_{M}^{T}\left(\mathcal{V}^{(M)}\left(\Psi^{(\leq M)}\right)-\mathcal{V}^{(M)}\left(\Psi^{(\leq h)}\right)\right)=e_{[h+1, M]}+\sum_{\sigma, \sigma^{\prime}} \int d \mathbf{x} d \mathbf{y} k_{\sigma \sigma^{\prime}}^{[h+1, M]}(\mathbf{x}, \mathbf{y}) \Psi_{\mathbf{x}, \sigma}^{+} \Psi_{\mathbf{y}, \sigma^{\prime}}^{-},
$$

where, denoting $g^{[h+1, M]}(\mathbf{x})=\sum_{h^{\prime}=h+1}^{M} g^{\left(h^{\prime}\right)}(\mathbf{x})$,

$$
\begin{aligned}
& e_{[h+1, M]}=e_{[h+1, M]}(\phi, A)=-\sum_{\sigma, \sigma^{\prime}} \int d \mathbf{x} d \mathbf{y}\left\{\left[K_{\sigma \sigma^{\prime}}^{1}(\mathbf{x}, \mathbf{y}) \phi_{\mathbf{x}}^{\sigma}+K_{\sigma \sigma^{\prime}}^{2}(\mathbf{x}, \mathbf{y}) A_{\mathbf{x}, \mathbf{y}}^{\sigma \sigma^{\prime}}+K_{\sigma \sigma^{\prime}}^{3}(\mathbf{x}, \mathbf{y})\right] .\right. \\
& \left.\cdot g_{\sigma^{\prime} \sigma}^{[h+1, M]}(\mathbf{0})+K_{\sigma \sigma^{\prime}}^{4}(\mathbf{x}, \mathbf{y})\left[g_{\sigma \sigma^{\prime}}^{[h+1, M]}(\mathbf{x}-\mathbf{y}) g_{\sigma^{\prime} \sigma}^{[h+1, M]}(\mathbf{y}-\mathbf{x})-g_{\sigma \sigma}^{[h+1, M]}(\mathbf{0}) g_{\sigma^{\prime} \sigma^{\prime}}^{[h+1, M]}(\mathbf{0})\right]\right\},
\end{aligned}
$$

and

$$
k_{\sigma \sigma^{\prime}}^{[h+1, M]}(\mathbf{x}, \mathbf{y})=2 U g_{\sigma \sigma^{\prime}}^{[h+1, M]}((0, \vec{x}-\vec{y})) \delta\left(x_{0}-y_{0}\right)\left[v_{\sigma \sigma^{\prime}}(\vec{x}-\vec{y})-\nu_{\sigma} \delta_{\sigma \sigma^{\prime}} \delta(\vec{x}-\vec{y})\right] .
$$

Therefore, it is natural to shrink all the branches of $\tau \in \widetilde{\mathcal{T}}_{M ; h, n}$ consisting of a subtree $\tau^{\prime} \subseteq \tau$, having root $r^{\prime}$ on scale $h^{\prime} \in[h, M]$ and only one endpoint on scale $M+1$, into a trivial subtree, rooted in $r^{\prime}$ and associated with a factor $\widetilde{\mathcal{V}}^{\left(h^{\prime}\right)}\left(\Psi^{\left(\leq h^{\prime}\right)}\right)$, which has the same structure as the right side of (5.39), with $E_{M}(\phi)$ replaced by $E_{h^{\prime}}(\phi, A)=E_{M}(\phi)+e_{\left[h^{\prime}+1, M\right]}(\phi, A)$, $K_{\sigma \sigma^{\prime}}^{3}(\mathbf{x}, \mathbf{y})$ replaced by $K_{h^{\prime}+1 ; \sigma \sigma^{\prime}}^{3}(\mathbf{x}, \mathbf{y}):=K_{\sigma \sigma^{\prime}}^{3}(\mathbf{x}, \mathbf{y})+k_{\sigma \sigma^{\prime}}^{\left[h^{\prime}+1, M\right]}(\mathbf{x}, \mathbf{y})$, and $\Psi$ replaced by $\Psi^{\left(\leq h^{\prime}\right)}$. Note that $k_{\sigma \sigma^{\prime}}^{[h+1, M]}(\mathbf{x}, \mathbf{y})$ is bounded proportionally to $U$, and decays faster than any power, uniformly in $M$, in the sense that

$$
\left\|k^{[h+1, M]}\right\|_{1, n}=\sup _{\sigma, \sigma^{\prime}} \int d \mathbf{x}\left|k_{\sigma \sigma^{\prime}}^{[h+1, M]}(\mathbf{x}, \mathbf{0})\right| \cdot|\mathbf{x}|^{n} \leq C_{n} 2^{-h}|U|, \quad \forall n \geq 0 .
$$

In particular, the $(1, n)$-norm of $K_{h^{\prime}}^{3}$ is bounded uniformly in $h^{\prime}$ and $M$, proportionally to $|U|$. By shrinking all the linear subtrees in the way explained above, we end up with an alternative representation of the effective potentials, which is based on a slightly modified tree expansion. The set of modified trees with $N$ endpoints contributing to $\mathcal{V}^{(h)}$ will be denoted by $\mathcal{T}_{M ; h, N}$; every $\tau \in \mathcal{T}_{M ; h, N}$ is characterized in the same way as the elements of $\widetilde{\mathcal{T}}_{M ; h, N}$, but for two features: (i) the endpoints of $\tau \in \mathcal{T}_{M ; h, N}$ are not necessarily on scale $M+1$; (ii) every endpoint $v$ of $\tau$ is attached to a non-trivial vertex on scale $h_{v}-1$ and is associated with the factor $\widetilde{\mathcal{V}}^{\left(h_{v}-1\right)}\left(\Psi^{\left(\leq h_{v}-1\right)}\right)$. See Fig.3. In terms of these modified trees, (5.56) is changed into

$$
\mathcal{V}^{(h)}\left(\Psi^{(\leq h)}\right)=\sum_{N=1}^{\infty} \sum_{\tau \in \mathcal{T}_{M ; h, N}} \mathcal{V}^{(h)}\left(\tau, \Psi^{(\leq h)}\right)
$$

where

$$
\mathcal{V}^{(h)}\left(\tau, \Psi^{(\leq h)}\right)=\frac{(-1)^{s-1}}{s !} \mathcal{E}_{h+1}^{T}\left[\mathcal{V}^{(h+1)}\left(\tau_{1}, \Psi^{(\leq h+1)}\right) ; \ldots ; \mathcal{V}^{(h+1)}\left(\tau_{s}, \psi^{(\leq h+1)}\right)\right]
$$

and, if $\tau$ is a trivial subtree with root on scale $k \in[h, M]$, then $\mathcal{V}^{(k)}\left(\tau, \Psi^{(\leq k)}\right)=\widetilde{\mathcal{V}}\left(\Psi^{(\leq k)}\right)$. 


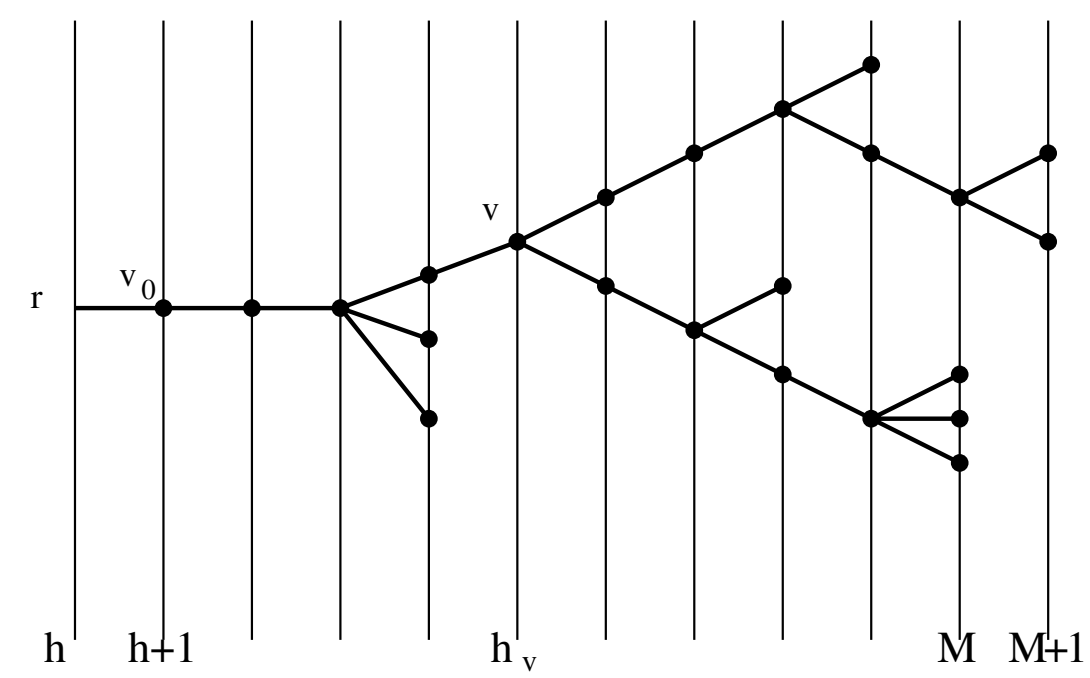

FIG. 3. A tree $\tau \in \mathcal{T}_{M ; h, N}$ with $N=9$ : the root is on scale $h$ and the endpoints are on scales $\leq M+1$.

Using its inductive definition Eq.(5.62), the right hand side of Eq.(5.61) can be further expanded (it is a sum of several contributions, differing for the choices of the field labels contracted under the action of the truncated expectations $\mathcal{E}_{h_{v}}^{T}$ associated with the vertices $v$ that are not endpoints), and in order to describe the resulting expansion we need some more definitions (allowing us to distinguish the fields that are contracted or not "inside the vertex $\left.v^{\prime \prime}\right)$.

We associate with any vertex $v$ of the tree a subset $P_{v}$ of $I_{v}$, the external fields of $v$. These subsets must satisfy various constraints. First of all, if $v$ is not an endpoint and $v_{1}, \ldots, v_{s_{v}}$ are the $s_{v} \geq 1$ vertices immediately following it (such that, in particular, $h_{v_{i}}=h_{v}+1$ ), then $P_{v} \subseteq \cup_{i} P_{v_{i}}$; if $v$ is an endpoint, $P_{v}=I_{v}$. If $v$ is not an endpoint, we shall denote by $Q_{v_{i}}$ the intersection of $P_{v}$ and $P_{v_{i}}$; this definition implies that $P_{v}=\cup_{i} Q_{v_{i}}$. The union $\mathcal{I}_{v}$ of the subsets $P_{v_{i}} \backslash Q_{v_{i}}$ is, by definition, the set of the internal fields of $v$, and is non empty if $s_{v}>1$. Given $\tau \in \mathcal{T}_{M ; h, N}$ and the set of field labels $I_{v}$ associated with the endpoints $v$ of $\tau$, there are many possible choices of the subsets $P_{v}$ associated with the vertices that are not endpoints, which are compatible with all the constraints. We shall denote by $\mathcal{P}_{\tau}$ the family of all these choices and by $\mathbf{P}$ the elements of $\mathcal{P}_{\tau}$. With these definitions, we can rewrite $\mathcal{V}^{(h)}\left(\tau, \Psi^{(\leq h)}\right)$ as:

$$
\mathcal{V}^{(h)}\left(\tau, \Psi^{(\leq h)}\right)=\sum_{\underline{\sigma}_{v_{0}}} \int d \underline{\mathbf{x}}_{v_{0}} \sum_{\mathbf{P} \in \mathcal{P}_{\tau}} K_{\tau, \mathbf{P}}^{(h+1)} \Psi_{P_{v_{0}}}^{(\leq h)}
$$


where $K_{\tau, \mathbf{P}}^{(h+1)}$ is defined inductively by the following equation, which is valid for any $v \in \tau$ that is not an endpoint,

$$
K_{\tau, \mathbf{P}}^{\left(h_{v}\right)}=\frac{1}{s_{v} !} \prod_{i=1}^{s_{v}}\left[K_{\tau_{i}, \mathbf{P}_{i}}^{\left(h_{v_{i}}\right)}\right] \mathcal{E}_{h_{v}}^{T}\left[\Psi_{P_{v_{1}} \backslash Q_{v_{1}}}^{\left(h_{v}\right)}, \ldots, \Psi_{P_{s_{v}} \backslash Q_{v_{s_{v}}}}^{\left(h_{v}\right)}\right] .
$$

Here $\tau_{1}, \ldots, \tau_{s_{v}}$ are the subtrees with root $v, v_{i}$ are their leftmost vertices (such that, in particular, $\left.h_{v_{i}}=h_{v}+1\right)$, and $\mathbf{P}_{i}=\left\{P_{w}, w \in \tau_{i}\right\}$. Moreover, if $v_{i}$ is an endpoint, then $K_{\tau_{i}, \mathbf{P}_{i}}^{\left(h_{v_{i}}\right)}=K_{v_{i}}$, with

$$
K_{v}=\left\{\begin{array}{lll}
E_{h_{v}-1}(\phi, A) & \text { if } & \rho_{v}=0 \\
K_{h_{v} ; \sigma_{v} \sigma_{v}^{\prime}}^{\rho_{v}}\left(\mathbf{x}_{v}, \mathbf{y}_{v}\right)\left[\phi_{\mathbf{x}_{v}}^{\sigma_{v}}\right]^{\delta_{v v}, 1}\left[A_{\mathbf{x}_{v}, \mathbf{y}_{v}}^{\sigma_{v} \sigma_{v}^{\prime}}\right]^{\delta_{\rho_{v}, 2}} & \text { if } & \rho_{v}>0
\end{array}\right.
$$

where $K_{h_{v} ; \sigma_{v} \sigma_{v}^{\prime}}^{\rho_{v}}$ should be identified with $K_{\sigma_{v} \sigma_{v}^{\prime}}^{\rho_{v}}$ in the case that $\rho_{v}=1,2,4$. Combining (5.61) with (5.63) and (5.64), and using the determinant representation of the truncated expectation, see (5.38), we finally get:

$$
\mathcal{V}^{(h)}\left(\Psi^{(\leq h)}\right)=E_{h}(\phi, A)+\sum_{N=1}^{\infty} \sum_{\tau \in \mathcal{T}_{M ; h, N}}^{*} \sum_{\underline{\sigma}_{0}} \int d \underline{\mathbf{x}}_{v_{0}} \sum_{\mathbf{P} \in \mathcal{P}_{\tau}} \sum_{T \in \mathbf{T}} W_{\tau, \mathbf{P}, T}^{(h)}\left(\underline{\mathbf{x}}_{v_{0}}, \underline{\sigma}_{v_{0}}\right) \Psi_{P_{v_{0}}}^{(\leq h)},
$$

where the $*$ on the sum over $\tau$ indicates the constraint that there are no endpoints of type 0 , and $\mathbf{T}$ is the set of the tree graphs on $\underline{\mathbf{x}}_{v_{0}}$ obtained by putting together an anchored tree graph $T_{v}$ for each non-trivial vertex $v$ and by adding a line (which is by definition the only element of $T_{v}$ ) for the couple of space-time points belonging to the set $\underline{\mathbf{x}}_{v}$ for each endpoint $v$. Moreover,

$$
W_{\tau, \mathbf{P}, T}\left(\mathbf{x}_{v_{0}}, \underline{\sigma}_{v_{0}}\right)=\alpha_{T}\left[\prod_{v \text { e.p. }} K_{v}\right] \prod_{\substack{\text { not } \\ \text { e.p. }}} \frac{1}{s_{v} !} \int d P_{T_{v}}\left(\mathbf{t}_{v}\right) \operatorname{det} G_{T_{v}}^{\left(h_{v}\right)}\left(\mathbf{t}_{v}\right) \prod_{\ell \in T_{v}} g_{\ell}^{\left(h_{v}\right)},
$$

where $\alpha_{T}$ is a sign and $G_{T_{v}}^{\left(h_{v}\right)}\left(\mathbf{t}_{v}\right)$ is a matrix analogous to the one defined after (5.38), with $g^{(M)}$ replaced by $g^{\left(h_{v}\right)}$. Note that $W_{\tau, \mathbf{P}, T}$ depends on $M$ only through: (i) the choice of the scale labels, and (ii) the (weak) $M$-dependence of the endpoints $v$ of type $\rho_{v}=3$, whose value is $K_{h_{v} ; \sigma_{v} \sigma_{v}^{\prime}}^{3}=K_{\sigma_{v} \sigma_{v}^{\prime}}^{3}+k_{\sigma_{v} \sigma_{v}^{\prime}}^{\left[h_{v}, M\right]}$, with $k_{\sigma_{v} \sigma_{v}^{\prime}}^{\left[h_{v}, M\right]}$ as in (5.59). From (5.66) and (5.67) we see that $\mathcal{V}^{(h)}(\Psi)$ can be rewritten as in (5.46), with $M-1$ replaced by $h$, and

$$
\begin{aligned}
& W_{2 n, s_{1}, s_{2}, \underline{\underline{\sigma}, \underline{\varepsilon}}}^{(h)}(\underline{\mathbf{x}}, \underline{\mathbf{y}}, \underline{\mathbf{z}})=\sum_{N \geq 1} \sum_{\tau \in \mathcal{T}_{M ; h, N}}^{* *} \sum_{\underline{\sigma}_{v_{0}}} \int d \underline{\mathbf{x}}_{v_{0}} \sum_{\substack{\mathbf{P} \in \mathcal{P}_{\tau}: \\
\left|P_{v_{0}}\right|=2 n}} \delta\left(I_{v_{0}}^{1}-I_{e x t}^{1}\right) \delta\left(I_{v_{0}}^{2}-I_{e x t}^{2}\right) \delta\left(P_{v_{0}}-P_{e x t}\right) \times \\
& \times\left[\prod_{v \text { e.p. }} K_{h_{v} ; \sigma_{v} \sigma_{v}^{\prime}}^{\rho_{v}}\left(\mathbf{x}_{v}, \mathbf{y}_{v}\right)\right] \sum_{T \in \mathbf{T}} \alpha_{T} \prod_{\substack{v \text { not } \\
\text { e.p. }}} \frac{1}{s_{v} !} \int d P_{T_{v}}\left(\mathbf{t}_{v}\right) \operatorname{det} G_{T_{v}}^{\left(h_{v}\right)}\left(\mathbf{t}_{v}\right) \prod_{\ell \in T_{v}} g_{\ell}^{\left(h_{v}\right)}
\end{aligned}
$$

where the $* *$ on the sum over $\tau$ indicates the constraint that $\tau$ has $s_{1}$ endpoints of type $1, s_{2}$ of type 2 , and no endpoints of type 0 . Note also that, in order for $\left|P_{v_{0}}\right|$ to be equal to $2 n$, the 
number of endpoints of type 3 and 4 must be $\geq n-1$, that is $N \geq s_{1}+s_{2}+n-1$. Moreover, $I_{\text {ext }}^{1}=\left(\left(\mathbf{x}_{1}, \sigma_{1}\right), \ldots,\left(\mathbf{x}_{s_{1}}, \sigma_{s_{1}}\right)\right), I_{\text {ext }}^{2}=\left(\left(\mathbf{x}_{s_{1}+1}, \mathbf{y}_{s_{1}+1}, \sigma_{s_{1}+1}, \sigma_{s_{1}+1}^{\prime}\right), \ldots,\left(\mathbf{x}_{s_{2}}, \mathbf{y}_{s_{2}}, \sigma_{s_{2}}, \sigma_{s_{2}}^{\prime}\right)\right)$, $P_{\text {ext }}=\left(\left(\mathbf{z}_{1}, \sigma_{1}^{\prime \prime}, \varepsilon_{1}\right), \ldots,\left(\mathbf{z}_{2 n}, \sigma_{2 n}^{\prime \prime}, \varepsilon_{2 n}\right)\right)$, and the functions $\delta\left(I_{v_{0}}^{1}-I_{\text {ext }}^{1}\right)$, etc, are shorthands of products of delta functions, in the same sense as $\delta\left(P-P_{\text {ext }}\right)$ in (5.47). Using the explicit expression (5.68), we obtain a bound analogous to (5.48):

$$
\begin{aligned}
& \frac{1}{\beta L^{2}} \int d \mathbf{x} d \mathbf{y} d \mathbf{z}\left|W_{2 n, s_{1}, s_{2}, \underline{\sigma}, \underline{\varepsilon}}^{(h)}(\underline{\mathbf{x}}, \underline{\mathbf{y}}, \underline{\mathbf{z}})\right| \leq \\
& \leq \sum_{\substack{N \geq 1: \\
N \geq s_{1}+s_{2}+n-1}} C^{N} \sum_{\tau \in \mathcal{T}_{M ; h, N}}^{* *} \sum_{\substack{\mathbf{P} \in \mathcal{P}_{\tau}: \\
\left|P_{v_{0}}\right|=2 n}}\left[\prod_{v \text { e.p. }}\left\|K^{\rho_{v}}\right\|_{1}\right] \sum_{T \in \mathbf{T}}\left[\prod_{\substack{v \text { not } \\
\text { e.p. }}} \frac{1}{s_{v} !}\left\|\operatorname{det} G_{T_{v}}^{\left(h_{v}\right)}\right\|_{\infty} \prod_{\ell \in T_{v}}\left\|g_{\ell}^{\left(h_{v}\right)}\right\|_{1}\right] .
\end{aligned}
$$

Now: (i) the contribution of the endpoints is bounded as $\left\|K^{\rho_{v}}\right\|_{1} \leq C|U|^{\delta_{\rho_{v}, 3}+\delta_{\rho_{v}, 4}}$, (ii) the 1-norm of the propagators is bounded as in (5.26), that is $\left\|g_{\ell}^{\left(h_{v}\right)}\right\|_{1} \leq C \delta_{\mu}^{-3} 2^{-h}$, and (iii) the determinant, recalling the Gram representation of the propagator (5.27), can be bounded by using the Gram-Hadamard inequality (5.50) in a way analogous to (5.51), that is

$$
\left\|\operatorname{det} G_{T_{v}}^{\left(h_{v}\right)}\right\|_{\infty} \leq C^{\sum_{i=1}^{s_{v}}\left|P_{v_{i}}\right|-\left|P_{v}\right|-2\left(s_{v}-1\right)}
$$

where $v_{1}, \ldots, v_{s_{v}}$ are the vertices immediately following $v$ on $\tau$. Plugging these bounds into (5.69), and using the fact that $\sum_{v \text { not. e.p. }}\left(\sum_{i=1}^{s_{v}}\left|P_{v_{i}}\right|-\left|P_{v}\right|\right) \leq 4\left(N-s_{1}-s_{2}\right)$, we obtain

$$
\sum_{\substack{N \geq 1: \\ N \geq s_{1}+s_{2}+n-1}} C^{N}|U|^{N-s_{1}-s_{2}} \sum_{\tau \in \mathcal{T}_{M ; h, N}}^{* *} \sum_{\substack{\mathbf{P} \in \mathcal{P}_{\tau}: \\\left|P_{v_{0}}\right|=2 n}} \sum_{T \in \mathbf{T}}\left[\prod_{\substack{v \text { not } \\ \text { e.p. }}} \frac{1}{s_{v} !}\left(C \delta_{\mu}^{-3} 2^{-h_{v}}\right)^{s_{v}-1}\right] .
$$

Using the following relation, which can be easily proved by induction,

$$
\sum_{\substack{v \text { not } \\ \text { e.p. }}} h_{v}\left(s_{v}-1\right)=h(N-1)+\sum_{\substack{v \text { not } \\ \text { e.p. }}}\left(h_{v}-h_{v^{\prime}}\right)(n(v)-1)
$$

where $v^{\prime}$ is the vertex immediately preceding $v$ on $\tau$ and $n(v)$ the number of endpoints following $v$ on $\tau$, we find that Eq.(5.71) can be rewritten as

$$
\sum_{\substack{N \geq 1: \\ N \geq s_{1}+s_{2}+n-1}} \sum_{\tau \in \mathcal{T}_{M ; h, N}}^{* *} \sum_{\substack{\mathbf{P} \in \mathcal{P}_{\tau}: \\ \mid P_{v_{0}}=2 n}} \sum_{T \in \mathbf{T}} C^{N} \delta_{\mu}^{-3(N-1)}|U|^{N-s_{1}-s_{2}} 2^{-h(N-1)}\left[\prod_{\substack{v \text { not. } \\ \text { e.p. }}} \frac{1}{s_{v} !} 2^{\left(h_{v}-h_{v^{\prime}}\right)(n(v)-1)}\right] .
$$

where, by construction, if $N>1$, then $n(v)>1$ for any vertex $v$ of $\tau \in \mathcal{T}_{M ; h, N}$ that is not an endpoint (simply because every endpoint $v$ of $\tau$ is attached to a non-trivial vertex on scale $h_{v}-1$, see the discussion after (5.59) and item (ii) after (5.60)). If $N=1$, the only tree contributing to the sum in (5.73) is trivial, with four possible type labels attached to the endpoint. The corresponding contribution to (5.73) is (const.) $|U|^{\delta_{s_{1}+s_{2}, 0}}$. The contribution to (5.73) from the terms with $N \geq 2$ can be bounded as follows: first of all, the number of 
terms in $\sum_{T \in \mathbf{T}}$ is bounded by $C^{N} \prod_{v}$ not e.p. $s_{v}$ ! (see, e.g., [32, appendix A.3.3]); moreover, $\left|P_{v}\right| \leq 4 n(v)$ and $n(v)-1 \geq \max \left\{1, \frac{n(v)}{2}\right\}$, so that $n(v)-1 \geq \frac{1}{2}+\frac{\left|P_{v}\right|}{16}$, and, therefore,

$$
\begin{aligned}
\frac{1}{\beta L^{2}} \int d \mathbf{x} d \mathbf{y} d \mathbf{z} \mid & \left.W_{2 n, s_{1}, s_{2}, \underline{\sigma}, \underline{\varepsilon}}^{(h)}(\underline{\mathbf{x}}, \underline{\mathbf{y}}, \underline{\mathbf{z}})\left|\leq \sum_{\substack{N \geq 1: \\
N \geq s_{1}+s_{2}+n-1}} C^{N} \delta_{\mu}^{-3(N-1)}\right| U\right|^{N-s_{1}-s_{2}} 2^{-h(N-1)} \times \\
& \times \sum_{\tau \in \mathcal{T}_{M ; h, N}}^{* *}\left(\prod_{\text {not e.p. }} 2^{-\frac{1}{2}\left(h_{v}-h_{v^{\prime}}\right)}\right) \sum_{\substack{\mathbf{P} \in \mathcal{P}_{\tau}: \\
\left|P_{v_{0}}\right|=2 n}}\left(\prod_{v \text { not }} 2^{-\left|P_{v}\right| / 16}\right)
\end{aligned}
$$

Now, the sums over $\tau$ and $\mathbf{P}$ in the second line can be both bounded by (const. $)^{N}$, see [32, Lemma A.2 in appendix A.1 and appendix A.6.1], which implies the uniform analyticity of the kernels of the effective potentials on scale $h$, for all $-1 \leq h<M$, provided $U$ is small enough, namely $|U| \leq$ (const.) $\delta_{\mu}^{3}$. Note that the regularized free energy and correlation functions are nothing but the constant part and the kernels of the effective potential with $h=-1$. Therefore, the regularized free energy is analytic in $U$, uniformly in $\beta, L, M$. Similarly, the regularized correlation functions are uniformly analytic and satisfy (5.23), uniformly in $\beta, L, M$, for $\underline{m}=\underline{0}$ and $|U|$ small enough. The proof of (5.23) for general choices of $\underline{m}$ follows similarly, by combining the previous strategy with the idea of decomposing the factors $\left|\mathbf{x}_{i}-\mathbf{x}_{j}\right|$ along the tree $T$, as in (5.53) and following discussion. This concludes the proof of (5.23) and of Lemma 5.2.

\section{Proof of Proposition 4.1}

We are left with proving the existence of the limit as $\beta, L, M \rightarrow \infty$ of the regularized free energy and correlation functions. In order to prove it, we show that these regularized functions form a Cauchy sequence. Let us start by showing that, for fixed $\beta, L$, and $M^{\prime}>M$, for all $0<\theta<1$, there exists $C_{\theta}>0$ such that

$$
\left\|K_{m, n}^{\beta, L, M}-K_{m, n}^{\beta, L, M^{\prime}}\right\|_{1, r} \leq C_{\theta} 2^{-\theta M}
$$

where

$$
\left\|K_{m, n}^{\beta, L, M}\right\|_{1, r}=\frac{1}{\beta L^{2}} \sup _{\underline{\sigma}} \sup _{\substack{\underline{m}: \\|\underline{m}|=r}} \int_{\Lambda_{\beta, L}^{m+n}} d \underline{\mathbf{x}} \sum_{\underline{\vec{y} \in \Lambda_{L}^{m}}}\left|K^{\beta, L, M}\left(\mathbf{x}_{1}, \vec{y}_{1}, \sigma_{1}, \sigma_{1}^{\prime} ; \ldots ; \mathbf{x}_{m+n}, \sigma_{m+n}\right)\right| d_{\underline{m}}(\underline{\mathbf{x}}, \underline{\vec{y}}) .
$$

As already remarked above, the regularized correlation function are the kernels of the effective potential on scale -1 . Therefore, both $K^{\beta, L, M}$ and $K^{\beta, L, M^{\prime}}$ can be expressed in terms of the tree expansion described above. As already remarked after (5.67), the expansions for $K^{\beta, L, M}$ and $K^{\beta, L, M^{\prime}}$ differ among each other only because of: (i) the choice of the scale labels (the trees contributing to $K^{\beta, L, M}$, resp. $K^{\beta, L, M^{\prime}}$, have endpoints on scales $\leq M+1$, resp. $\leq M^{\prime}+1$ ); (ii) the dependence on the ultraviolet cutoff of the endpoints of type 3 , whose value 
is $K_{h_{v} ; \sigma_{v} \sigma_{v}^{\prime}}^{3}=K_{\sigma_{v} \sigma_{v}^{\prime}}^{3}+k_{\sigma_{v} \sigma_{v}^{\prime}}^{\left[h_{v}, M\right]}$ in the trees contributing to $K^{\beta, L, M}$, and similarly for $K^{\beta, L, M^{\prime}}$. This means that the difference $K^{\beta, L, M}-K^{\beta, L, M^{\prime}}$ can be expressed as a sum over trees whose root is on scale -1 and: (A) either there is at least one endpoint on scale $>M+1$, or (B) there is one endpoint of type 3 associated with a difference $k_{\sigma_{v} \sigma_{v}^{\prime}}^{\left[h_{v}, M\right]}-k_{\sigma_{v} \sigma_{v}^{\prime}}^{\left[h_{v}, M^{\prime}\right]}=k_{\sigma_{v} \sigma_{v}^{\prime}}^{\left[M+1, M^{\prime}\right]}$.

The contributions from the case (A) can be bounded as in (5.73), with $h=-1$ and the extra constraint that there is at least one endpoint on scale $>M+1$. This means that the factor $\prod_{\text {e.p. }} 2^{\left(h_{v}-h_{v^{\prime}}\right)(n(v)-1)}$ is smaller than $2^{-M}$. The idea is then to split this term into two factors, in the form $\left[\prod_{\text {e.p. }}\right.$ not $\left.2^{\theta\left(h_{v}-h_{v^{\prime}}\right)(n(v)-1)}\right] \times\left[\prod_{\text {e.p. }}\right.$ not $\left.2^{(1-\theta)\left(h_{v}-h_{v^{\prime}}\right)(n(v)-1)}\right]$. The first factor is smaller than $2^{-\theta M}$, while the sum over the scale and field labels of the second factor can be bounded exactly in the same away as it was explained after (5.74).

Concerning case (B), it is enough to note that the norm of $k_{\sigma_{v} \sigma_{v}^{\prime}}^{\left[M+1, M^{\prime}\right]}$ is proportional to $2^{-M}$, see (5.60), which implies that the overall contribution from these trees is smaller than the norm of $K^{\beta, L, M}$ by a factor $2^{-M}$.

In conclusion, we obtain (5.75). By Vitali's uniform convergence theorem for analytic functions, we conclude that the limit as $M \rightarrow \infty$ of any weighted integral of the regularized correlations (with weights growing at most polynomially at large space-time differences, and the integral normalized by $\left.1 /\left(\beta L^{2}\right)\right)$ is analytic, and its Taylor coefficients are the $M \rightarrow \infty$ limit of the coefficients of the regularized correlations. Analogously, one proves the same for the correlation functions at fixed space-time positions. Moreover, the same argument is valid for the limit as $\beta, L \rightarrow \infty$, see [34, appendix D] for a thorough discussion of this limit. Of course, the same claims are valid for the regularized free energy, too.

Finally, the statement of proposition 4.1 follows from the remark that that the correlation functions in momentum space can be expressed as the Fourier transforms of their space-time counterparts, and that their derivatives of order $r$ are controlled by the $(1, r)$ norms $(5.76)$ of the space-time correlation functions, which are finite and bounded uniformly in $\beta, L, M$, as we just proved.

\section{RECONSTRUCTION OF THE REAL-TIME KUBO FORMULA}

In this section we prove theorem 3.1, which says that the Kubo formula (3.11), which can be expressed as an imaginary-time integral of the current-current correlation, can be analytically continued to real times. In other words, we rigorously prove the validity of the Wick rotation for the Kubo conductivity of the class of systems under investigation. In the language of Quantum Field Theory, we prove a reconstruction theorem for the conductivity matrix of weakly interacting gapped fermionic systems.

The proof of theorem 3.1 is based on the existence of the real-time correlation functions in the infinite volume and zero temperature limits, as well as on the decay of the complex time correlations, as summarized in the following proposition. 
Proposition 6.1 [Properties of the current-current correlations at complex times] Let $J_{i}(z):=e^{z \mathcal{H}_{L}} J_{i} e^{-z \mathcal{H}_{L}}, z \in \mathbb{C}$, with $J_{i}$ defined in (2.15)-(2.17) and following lines. Under the same assumptions as theorem 2.1, and if in addition $U \in \mathbb{R}$, the following is true.

- (i) Let $z \in \mathbb{C}^{+}=\{z \in \mathbb{C} \mid \operatorname{Re} z>0\}$. Then, the limit

$$
\lim _{\beta \rightarrow \infty} \lim _{L \rightarrow \infty} \frac{1}{L^{2}}\left\langle J_{i}(z) J_{j}(0)\right\rangle_{\beta, L}=:\left\langle J_{i}(z) J_{j}(0)\right\rangle_{\infty}
$$

exists, and it is analytic in $z \in \mathbb{C}^{+}$. Moreover, it decays faster than any power in $\operatorname{Re} z$, i.e.,

$$
\left|\left\langle J_{i}(z) J_{j}(0)\right\rangle_{\infty}\right| \leq \frac{C_{M}}{1+\left(\delta_{\mu} \operatorname{Re} z\right)^{M}}, \quad z \in \mathbb{C}^{+},
$$

for all $M \geq 0$ and a suitable $C_{M}>0$.

- (ii) Let $t \in \mathbb{R}$. Then, the limit

$$
\lim _{\beta \rightarrow \infty} \lim _{L \rightarrow \infty} \frac{1}{L^{2}}\left\langle\left[J_{i}(i t), J_{j}(0)\right]\right\rangle_{\beta, L}=:\left\langle\left[J_{i}(i t), J_{j}(0)\right]\right\rangle_{\infty}
$$

exists and is finite, uniformly in $t$.

Proof of proposition 6.1. To begin with, let us prove item (i). The starting point is to notice that the positive temperature, finite volume current-current correlation

$$
\left\langle J_{i}(z) J_{j}(0)\right\rangle_{\beta, L}=\frac{\operatorname{Tr} e^{-\beta\left(\mathcal{H}_{L}-\mu \mathcal{N}_{L}\right)} e^{z \mathcal{H}_{L}} J_{i} e^{-z \mathcal{H}_{L}} J_{j}}{\operatorname{Tr} e^{-\beta\left(\mathcal{H}_{L}-\mu \mathcal{N}_{L}\right)}}
$$

is entire in z. By the Cauchy-Schwarz inequality and the cyclicity of the trace, recalling that $U \in \mathbb{R}$, we get:

$$
\begin{aligned}
\left|\left\langle J_{i}(z) J_{j}(0)\right\rangle_{\beta, L}\right| & \leq\left|\left\langle J_{i}(z / 2) J_{i}(z / 2)^{\dagger}\right\rangle_{\beta, L}\right|^{1 / 2}\left|\left\langle J_{j}(-z / 2)^{\dagger} J_{j}(-z / 2)\right\rangle_{\beta, L}\right|^{1 / 2} \\
& =\left|\left\langle J_{i}(\operatorname{Re} z) J_{i}(0)\right\rangle_{\beta, L}\right|^{1 / 2}\left|\left\langle J_{j}(\operatorname{Re} z) J_{j}(0)\right\rangle_{\beta, L}\right|^{1 / 2} .
\end{aligned}
$$

Thus, for $0 \leq \operatorname{Re} z<\beta$, the right side of (6.5) can be estimated via (the proof of) proposition 4.1. This implies, in particular, that, if $0 \leq t<\beta$, the imaginary-time correlation function $L^{-2}\left\langle J_{i}(t) J_{i}(0)\right\rangle_{\beta, L}$ decays faster than any power in $|t|_{\beta}=\min _{n \mathbb{Z}}|t+n \beta|$, uniformly in $\beta, L$ (see Lemma 5.2 and section $5 \mathrm{C}$ ). Therefore, (6.5) implies that, for every fixed $z \in \overline{\mathbb{C}^{+}}$, there exists $\beta_{0}$ such that, for $\beta>\beta_{0}$,

$$
\frac{1}{L^{2}}\left|\left\langle J_{i}(z) J_{j}(0)\right\rangle_{\beta, L}\right| \leq \frac{C_{M}}{1+\left(\delta_{\mu} \operatorname{Re} z\right)^{M}},
$$

for all $M \geq 0$ and a suitable $C_{M}>0$, independent of $\beta, L, z$. Moreover, the proof of proposition 4.1 implies that the limit

$$
\lim _{\beta \rightarrow \infty} \lim _{L \rightarrow \infty} \frac{1}{L^{2}}\left\langle J_{i}(t) J_{j}(0)\right\rangle_{\beta, L}
$$


exists, for all $t \geq 0$. Therefore, by Vitali's theorem on the convergence of holomorphic functions, we conclude that the limit

$$
\lim _{\beta \rightarrow \infty} \lim _{L \rightarrow \infty} \frac{1}{L^{2}}\left\langle J_{i}(z) J_{j}(0)\right\rangle_{\beta, L}=:\left\langle J_{i}(z) J_{j}(0)\right\rangle_{\infty}
$$

exists and is analytic in $z$ in the whole open right half-plane $\mathbb{C}^{+}$. Moreover, the convergence to the limit is uniform on any compact subset of $\mathbb{C}^{+}$. By (6.6), the limit satisfies (6.2), which concludes the proof of item (i).

Let us now prove (ii). By using the translational invariance of the Gibbs state, we rewrite (recalling (2.16)-(2.17)):

$$
\frac{1}{L^{2}}\left\langle\left[J_{i}(i t), J_{j}(0)\right]\right\rangle_{\beta, L}=-\frac{1}{4} \sum_{\vec{x}, \vec{y}, \vec{z} \in \Lambda_{L}} \sum_{\sigma_{1}, \ldots, \sigma_{4} \in I}\left(\vec{x}_{\sigma_{1}}-\vec{y}_{\sigma_{2}}\right)_{i}\left(\overrightarrow{0}_{\sigma_{3}}-\vec{z}_{\sigma_{4}}\right)_{j}\left\langle\left[\left(J_{\vec{x} \vec{y}}^{\sigma_{1} \sigma_{2}}\right)(i t), J_{\overrightarrow{0} \vec{z}}^{\sigma_{3} \sigma_{4}}\right]\right\rangle_{\beta, L},
$$

where, again, $A(i t)=e^{i \mathcal{H}_{L} t} A e^{-i \mathcal{H}_{L} t}$. Now, the summand in the right side of (6.9) is absolutely summable, uniformly in $\beta, L$. This can be proven using Lieb-Robinson bounds; see, e.g., [45, 49, 50] for a derivation of these bounds for quantum spin systems and [19] for an extension to fermionic systems. The key result is (see, e.g., [49, Theorem 2.1], or [19, Theorem 3.1]):

$$
\left\|\left[\left(J_{\vec{x} \vec{y}}^{\sigma_{1} \sigma_{2}}\right)(i t), J_{\overrightarrow{0} \vec{z}}^{\sigma_{3} \sigma_{4}}\right]\right\| \leq\left\|J_{\vec{x} \vec{y}}^{\sigma_{1} \sigma_{2}}\right\|\left\|J_{\overrightarrow{0} \vec{z}}^{\sigma_{3} \sigma_{4}}\right\| \frac{C_{M} e^{v|t|}}{[1+\operatorname{dist}(\{\vec{x}, \vec{y}\},\{\overrightarrow{0}, \vec{z}\})]^{M}}
$$

for all $M \geq 0$ and suitable constants $C_{M}$ and $v$, independent of $\beta, L, t$. Here $\|\cdot\|$ denotes the operator norm. By using the fact that $\left\|J_{\vec{x} \vec{y}}^{\sigma_{1} \sigma_{2}}\right\| \leq 2\left\|H_{\sigma_{1} \sigma_{2}}(\vec{x}-\vec{y})\right\|$, which decays faster than any power in $|\vec{x}-\vec{y}|$, we see that the sum in the right side of (6.9) is absolutely convergent, uniformly in $\beta, L$.

Therefore, in order to prove the existence of the limit of (6.9) as $\beta, L \rightarrow \infty$, it is enough to prove, term by term, the existence of the limit of the summands in the right side. The proof of this fact is a straightforward consequence of the existence of the infinite volume dynamics (see $[19,49,50])$ and of the existence of the $\beta, L \rightarrow \infty$ limit of the Gibbs state. In appendix C, we reproduce this proof; that is, we prove that the $\operatorname{limit}_{\lim } \lim _{\beta \rightarrow \infty} \lim _{L \rightarrow \infty}\left\langle\left[\left(J_{\vec{x} \vec{y}}^{\sigma_{1} \sigma_{2}}\right)(i t), J_{\overrightarrow{0} \vec{z}}^{\sigma_{3} \sigma_{4}}\right]\right\rangle_{\beta, L}$ exists, thus concluding the proof of the existence of the limit in item (ii). The uniform boundedness of the limit is a consequence of (6.5)-(6.6).

We are now in the position of proving theorem 3.1.

Proof of theorem 3.1. We start from the very definition of imaginary-time conductivity (3.11), that is $\bar{\sigma}_{i j}(U)=-\lim _{\omega \rightarrow 0^{+}}(A \omega)^{-1}\left[\widehat{K}_{i j}(\omega, \overrightarrow{0})-\widehat{K}_{i j}(0, \overrightarrow{0})\right]$, where

$$
\widehat{K}_{i j}(\omega, \overrightarrow{0})=\lim _{\beta \rightarrow \infty} \lim _{L \rightarrow \infty} \frac{1}{\beta L^{2}} \int_{0}^{\beta} d t \int_{0}^{\beta} d t^{\prime} e^{-i \omega\left(t-t^{\prime}\right)}\left\langle\mathbf{T} J_{i}(t) J_{j}\left(t^{\prime}\right)\right\rangle_{\beta, L}
$$


Note that there is no semicolon between the two current operators in the right side (that is, the expectation is untruncated): the reason is that the Gibbs average of $J_{i}$ vanishes, simply because $J_{i}$ is proportional to the commutator of $\mathcal{H}_{L}$ with $X_{i}$. With some abuse of notation, we denoted by the same symbol the frequency $\omega$ in the two sides of the equation. However, it should be recalled that the (Matsubara) frequency in the right side is an integer multiple of $2 \pi / \beta$, i.e., it should be understood as being equal to $\omega_{n}=(2 \pi / \beta) n$, with $\omega_{n} \rightarrow \omega$ as $\beta \rightarrow \infty$.

We start by analyzing and suitably rewriting $\widehat{K}_{i j}(\omega, \overrightarrow{0})$, with $\omega>0$. By the cyclicity of the trace and the fact that $\omega_{n}$ is an integer multiple of $(2 \pi / \beta)$, we can rewrite

$$
\frac{1}{\beta} \int_{0}^{\beta} d t \int_{0}^{\beta} d t^{\prime} e^{-i \omega_{n}\left(t-t^{\prime}\right)}\left\langle\mathbf{T} J_{i}(t) J_{j}\left(t^{\prime}\right)\right\rangle_{\beta, L}=\int_{-\beta / 2}^{\beta / 2} d t e^{-i \omega_{n} t}\left\langle\mathbf{T} J_{i}(t) J_{j}(0)\right\rangle_{\beta, L},
$$

so that

$$
\widehat{K}_{i j}(\omega, \overrightarrow{0})=\lim _{\beta \rightarrow \infty} \lim _{L \rightarrow \infty} \frac{1}{L^{2}} \int_{-\beta / 2}^{\beta / 2} d t e^{-i \omega_{n} t}\left\langle\mathbf{T} J_{i}(t) J_{j}(0)\right\rangle_{\beta, L}
$$

Recalling that $L^{-2}\left\langle\mathbf{T} J_{i}(t) J_{j}(0)\right\rangle_{\beta, L}$ decays faster than any power in $\|t\|_{\beta}$, uniformly in $\beta, L$, and that it converges as $\beta, L \rightarrow \infty$, we find that, for any $T>0$,

$$
\widehat{K}_{i j}(\omega, \overrightarrow{0})=\int_{-T}^{T} d t e^{-i \omega t}\left\langle\mathbf{T} J_{i}(t) J_{j}(0)\right\rangle_{\infty}+R_{T}(\omega)
$$

where

$$
\left|R_{T}(\omega)\right| \leq \frac{C_{M}}{1+\left(\delta_{\mu} T\right)^{M}}
$$

for all $M \geq 0$ and a suitable $C_{M}>0$, independent of $T$ and $\omega$. Therefore,

$$
\widehat{K}_{i j}(\omega, \overrightarrow{0})=\lim _{T \rightarrow \infty} \int_{-T}^{T} d t e^{-i \omega t}\left\langle\mathbf{T} J_{i}(t) J_{j}(0)\right\rangle_{\infty}
$$

We rewrite:

$$
\int_{-T}^{T} e^{-i \omega t}\left\langle\mathbf{T} J_{i}(t) J_{j}(0)\right\rangle_{\infty}=\int_{0}^{T} e^{-i \omega t}\left\langle J_{i}(t) J_{j}(0)\right\rangle_{\infty}+\int_{-T}^{0} e^{-i \omega t}\left\langle J_{j}(0) J_{i}(t)\right\rangle_{\infty} .
$$

We study the two integrals in the right side separately, starting from the first. Recall that the integrand is analytic in $\mathbb{C}^{+}$: therefore, by Cauchy theorem, the integral along any closed path in $\mathbb{C}^{+}$is identically zero. We choose the closed path consisting of the union of: the segment $[\varepsilon, T]$ on the real line ( $\varepsilon$ being a small positive number, to be eventually sent to zero), directed from left to right; the quarter circle of radius $T-\varepsilon$ centered in $\varepsilon$, connecting the point $T$ with the point $-i(T-\varepsilon)+\varepsilon$, in the clockwise direction; and the vertical segment connecting $-i(T-\varepsilon)+\varepsilon$ with $\varepsilon$, in the upwards direction. We thus rewrite:

$$
\begin{aligned}
\int_{0}^{T} d t e^{-i \omega t}\left\langle J_{i}(t) J_{j}(0)\right\rangle_{\infty} & =\lim _{\varepsilon \rightarrow 0}\left[-i \int_{-T+\varepsilon}^{0} d t e^{\omega(t-i \varepsilon)}\left\langle J_{i}(i t+\varepsilon) J_{j}(0)\right\rangle_{\infty}\right. \\
& \left.+i(T-\varepsilon) \int_{-\pi / 2}^{0} d \theta e^{i \theta} e^{-i \omega\left(\varepsilon+(T-\varepsilon) e^{i \theta}\right)}\left\langle J_{i}\left(\varepsilon+(T-\varepsilon) e^{i \theta}\right) J_{j}(0)\right\rangle_{\infty}\right] .
\end{aligned}
$$


Recalling (6.2), we can bound the term in the second line by:

$T \lim _{\varepsilon \rightarrow 0} \int_{-\pi / 2}^{0} d \theta e^{\omega(T-\varepsilon) \sin \theta} \frac{C_{M}}{1+\left[\delta_{\mu}(\varepsilon+(T-\varepsilon) \cos \theta)\right]^{M}} \leq \frac{\pi}{4} C_{M} T\left[e^{-\omega T / \sqrt{2}}+\frac{1}{1+\left(\delta_{\mu} T / \sqrt{2}\right)^{M}}\right]$,

which tends to zero faster than any power as $T \rightarrow \infty$, for every $\omega>0$. Repeating the same argument for the second integral in the right side of (6.17), and plugging the result back into (6.16), we find that, for every $\omega>0$,

$$
\widehat{K}_{i j}(\omega, \overrightarrow{0})=-i \lim _{\varepsilon \rightarrow 0} \int_{-\infty}^{0} d t e^{\omega t}\left[\left\langle J_{i}(i t+\varepsilon) J_{j}(0)\right\rangle_{\infty}-\left\langle J_{j}(0) J_{i}(i t-\varepsilon)\right\rangle_{\infty}\right] .
$$

By adding and subtracting the expression in square brackets at $\varepsilon=0$, we get

$$
\widehat{K}_{i j}(\omega, \overrightarrow{0})=-i \int_{-\infty}^{0} d t e^{\omega t}\left\langle\left[J_{i}(i t), J_{j}(0)\right]\right\rangle_{\infty}+\lim _{\varepsilon \rightarrow 0} R(\omega, \varepsilon)
$$

where we used item (ii) of proposition 6.1, and we defined:

$$
R(\omega, \varepsilon)=\lim _{\beta \rightarrow \infty} \lim _{L \rightarrow \infty} \frac{-i}{L^{2}} \int_{-\infty}^{0} d t e^{\omega t}\left\langle\left[J_{i}(i t+\varepsilon)-J_{i}(i t)\right] J_{j}(0)-J_{j}(0)\left[J_{i}(i t-\varepsilon)-J_{i}(i t)\right]\right\rangle_{\beta, L} .
$$

The term $\left\langle\left[J_{i}(i t+\varepsilon)-J_{i}(i t)\right] J_{j}(0)\right\rangle_{\beta, L}$ can be bounded by rewriting it as:

$$
\begin{aligned}
\left\langle\left[J_{i}(i t+\varepsilon)-J_{i}(i t)\right] J_{j}(0)\right\rangle_{\beta, L} & =\int_{0}^{\varepsilon} d s\left\langle\frac{d}{d s} e^{(i t+s) \mathcal{H}_{L}} J_{i} e^{-(i t+s) \mathcal{H}_{L}} J_{j}\right\rangle_{\beta, L} \\
& =\int_{0}^{\varepsilon} d s\left\langle\left[\mathcal{H}_{L}, J_{i}(i t+s)\right] J_{j}\right\rangle_{\beta, L} .
\end{aligned}
$$

By proceeding as in the proof of proposition 6.1, via the analogues of (6.5)-(6.6), we obtain

$$
\left|\left\langle\left[J_{i}(i t+\varepsilon)-J_{i}(i t)\right] J_{j}(0)\right\rangle_{\beta, L}\right| \leq L^{2} C \varepsilon
$$

with $C>0$ independent of $\beta, L, \varepsilon$. The same estimate is valid for $\left\langle J_{j}(0)\left[J_{i}(i t-\varepsilon)-J_{i}(i t)\right]\right\rangle_{\beta, L}$. Plugging these estimates back into (6.22), we find that $|R(\omega, \varepsilon)| \leq C \varepsilon / \omega$, so that, using (6.21), we finally get that, for all $\omega>0$,

$$
\widehat{K}_{i j}(\omega, \overrightarrow{0})=-i \int_{-\infty}^{0} d t e^{\omega t}\left\langle\left[J_{i}(i t), J_{j}(0)\right]\right\rangle_{\infty}
$$

which is our final expression for $\widehat{K}_{i j}(\omega, \overrightarrow{0})$, with $\omega>0$.

Concerning $\widehat{K}_{i j}(0, \overrightarrow{0})$, in order to rewrite it conveniently, we use (4.4) with $n=2$ and $\alpha_{2}=j$ in the thermodynamic and zero temperature limits, which reads (denoting $\mathbf{p}=(\omega, \vec{p})$ )

$$
i \omega \widehat{K}_{0 j}(\mathbf{p})+p_{1} \widehat{K}_{1 j}(\mathbf{p})+p_{2} \widehat{K}_{2 j}(\mathbf{p})=\widehat{S}_{j}(\mathbf{p})
$$


If we derive this expression with respect to $p_{i}$ and then set $\mathbf{p}=\mathbf{0}$, we obtain

$$
\widehat{K}_{i j}(0, \overrightarrow{0})=\frac{\partial \widehat{S}_{j}}{\partial p_{i}}(\mathbf{0})=-\left\langle\left[\left[\mathcal{H}, X_{i}\right], X_{j}\right]\right\rangle_{\infty} .
$$

Using (6.25) and (6.27) in (3.11), we finally recognize that $\bar{\sigma}_{i j}(U)=(2.18)$, as desired.

Acknowledgements. We would like to thank Gian Michele Graf and Gianluca Panati for interesting discussions on the topological aspects of condensed matter physics, and Giuseppe Benfatto for useful comments. We gratefully acknowledge financial support from: the A*MIDEX project ( ${ }^{o}$ ANR-11-IDEX-0001-02) funded by the "Investissements d'Avenir" French Government program, managed by the French National Research Agency (A.G.); the PRIN National Grant Geometric and analytic theory of Hamiltonian systems in finite and infinite dimensions (A.G. and V. M.); the NCCR SwissMAP (M.P.).

\section{Appendix A: The non-interacting conductivity}

In this appendix we reproduce the well known result that, in the absence of interactions, and under the gap condition (2.13), the Kubo conductivity (3.11) reduces to the usual formula for the Chern number:

$$
\bar{\sigma}_{i j}(0)=i \int_{\mathcal{B}} \frac{d \vec{k}}{(2 \pi)^{2}} \operatorname{Tr} P_{-}(\vec{k})\left[\partial_{k_{i}} P_{-}(\vec{k}), \partial_{k_{j}} P_{-}(\vec{k})\right],
$$

where $P_{-}(\vec{k})$ is the projection onto the filled bands, defined after (2.13). In light of theorem 3.1 , the same is true for $\sigma_{i j}(0)$.

Our starting point consists in rewriting the infinite volume limit of the current operator defined in (2.15)-(2.17) in Fourier space:

$$
\vec{J}=i \int_{\mathcal{B}} \frac{d \vec{k}}{|\mathcal{B}|} \sum_{\sigma, \sigma^{\prime} \in I} \hat{\psi}_{\vec{k}, \sigma}^{+}\left[\left(i \nabla_{\vec{k}}+\vec{r}_{\sigma^{\prime}}-\vec{r}_{\sigma}\right) \hat{H}_{\sigma \sigma^{\prime}}^{(0)}(\vec{k})\right] \hat{\psi}_{\vec{k}, \sigma^{\prime}}^{-}
$$

The term $\vec{r}_{\sigma^{\prime}}-\vec{r}_{\sigma}$ can be reabsorbed by conjugating the Bloch Hamiltonian and the fermionic fields with a suitable unitary transformation: if we define $U(\vec{k})=\operatorname{diag}\left(e^{i \vec{k} \cdot \vec{r}_{1}}, \cdots, e^{i \vec{k} \cdot \vec{r}_{|I|}}\right)$, $\widetilde{H}^{(0)}(\vec{k})=U(\vec{k}) \hat{H}^{(0)}(\vec{k}) U(\vec{k})^{\dagger}, \widetilde{\psi}_{\vec{k}}^{-}=U(\vec{k}) \hat{\psi}_{\vec{k}}^{-}$, and $\widetilde{\psi}_{\vec{k}}^{+}=\hat{\psi}_{\vec{k}}^{+} U(\vec{k})^{\dagger}$, then the current in (A.2) can be rewritten as:

$$
\vec{J}=-\int_{\mathcal{B}} \frac{d \vec{k}}{|\mathcal{B}|} \sum_{\sigma, \sigma^{\prime} \in I} \widetilde{\psi}_{\vec{k}, \sigma}^{+}\left[\nabla_{\vec{k}} \widetilde{H}_{\sigma \sigma^{\prime}}^{(0)}(\vec{k})\right] \widetilde{\psi}_{\vec{k}, \sigma^{\prime}}^{-}
$$

Its imaginary-time evolution, $\overrightarrow{J_{t}}$, is obtained by replacing $\widetilde{\psi}_{\vec{k}, \sigma}^{ \pm}$by its imaginary-time evolution, $\widetilde{\psi}_{(t, \vec{k}), \sigma}^{ \pm}$. Note that $J_{i, t}$ is the same as (the infinite volume limit of) $\left.\tilde{J}_{i,(t, \vec{p})}\right|_{\vec{p}=\overrightarrow{0}}$, where $\tilde{J}_{i,(t, \vec{p})}$ was defined in (3.9). 
The conductivity matrix (3.11) at $U=0$ can then be re-expressed as:

$$
\begin{aligned}
\bar{\sigma}_{i j}(0)= & -\frac{1}{A} \lim _{\omega \rightarrow 0} \frac{\partial}{\partial \omega} \int_{\mathbb{R}} d t e^{-i \omega t} \int_{\mathcal{B}} \frac{d \vec{k}}{|\mathcal{B}|} \int_{\mathcal{B}} \frac{d \vec{k}^{\prime}}{|\mathcal{B}|} \sum_{\sigma_{1}, \ldots, \sigma_{4}} \times \\
& \times\left\langle\mathbf{T} \widetilde{\psi}_{(t, \vec{k}), \sigma_{1}}^{+} \partial_{k_{i}} \widetilde{H}_{\sigma_{1} \sigma_{2}}^{(0)}(\vec{k}) \widetilde{\psi}_{(t, \vec{k}), \sigma_{2}}^{-} ; \widetilde{\psi}_{\left(0, \vec{k}^{\prime}\right), \sigma_{3}}^{+} \partial_{k_{j}} \widetilde{H}_{\sigma_{3} \sigma_{4}}^{(0)}\left(\vec{k}^{\prime}\right) \widetilde{\psi}_{\left(0, \vec{k}^{\prime}\right), \sigma_{4}}^{-}\right\rangle^{(0)},
\end{aligned}
$$

where $\langle\cdot\rangle^{(0)}=\lim _{\beta \rightarrow \infty} \lim _{L \rightarrow \infty}\langle\cdot\rangle_{\beta, L}^{(0)}$. The expectation in the second line can be evaluated via the Wick rule, so that

$$
\bar{\sigma}_{i j}(0)=\frac{-i}{A} \int_{\mathcal{B}} \frac{d \vec{k}}{|\mathcal{B}|} \int_{\mathbb{R}} d t t \operatorname{Tr}\left\{\widetilde{g}(-t, \vec{k}) \partial_{k_{i}} \widetilde{H}^{(0)}(\vec{k}) \widetilde{g}(t, \vec{k}) \partial_{k_{j}} \widetilde{H}^{(0)}(\vec{k})\right\}
$$

where the trace is over the $\sigma$ indices, and

$$
\widetilde{g}(t, \vec{k})=e^{-t\left(\widetilde{H}^{(0)}(\vec{k})-\mu\right)}\left[\mathbb{1}(t>0) \widetilde{P}_{+}(\vec{k})-\mathbb{1}(t \leq 0) \widetilde{P}_{-}(\vec{k})\right],
$$

with $\widetilde{P}_{-}(\vec{k})=U(\vec{k}) P_{-}(\vec{k}) U(\vec{k})^{\dagger}$, and $\widetilde{P}_{+}(\vec{k})=1-\widetilde{P}_{-}(\vec{k})$. Plugging (A.6) into (A.5), and noting that $A|\mathcal{B}|=(2 \pi)^{2}$, we find

$$
\begin{aligned}
\bar{\sigma}_{i j}(0)= & i \int_{\mathcal{B}} \frac{d \vec{k}}{(2 \pi)^{2}}\left[\int_{0}^{\infty} d t t \operatorname{Tr}\left\{e^{t \widetilde{H}^{(0)}(\vec{k})} \widetilde{P}_{-}(\vec{k}) \partial_{k_{i}} \widetilde{H}^{(0)}(\vec{k}) e^{-t \widetilde{H}^{(0)}(\vec{k})} \widetilde{P}_{+}(\vec{k}) \partial_{k_{j}} \widetilde{H}^{(0)}(\vec{k})\right\}\right. \\
& \left.\quad+\int_{-\infty}^{0} d t t \operatorname{Tr}\left\{e^{t \widetilde{H}^{(0)}(\vec{k})} \widetilde{P}_{+}(\vec{k}) \partial_{k_{i}} \widetilde{H}^{(0)}(\vec{k}) e^{-t \widetilde{H}^{(0)}(\vec{k})} \widetilde{P}_{-}(\vec{k}) \partial_{k_{j}} \widetilde{H}^{(0)}(\vec{k})\right\}\right] \\
\equiv & i \int_{\mathcal{B}} \frac{d \vec{k}}{(2 \pi)^{2}}\left[\Sigma_{i j}(\vec{k})-\Sigma_{j i}(\vec{k})\right]
\end{aligned}
$$

where $\Sigma_{i j}(\vec{k})=\int_{0}^{\infty} d t t f_{i j}(t, \vec{k})$, with

$$
f_{i j}(t, \vec{k})=\operatorname{Tr}\left\{e^{t \widetilde{H}^{(0)}(\vec{k})} \widetilde{P}_{-}(\vec{k}) \partial_{k_{i}} \widetilde{H}^{(0)}(\vec{k}) e^{-t \widetilde{H}^{(0)}(\vec{k})} \widetilde{P}_{+}(\vec{k}) \partial_{k_{j}} \widetilde{H}^{(0)}(\vec{k})\right\} .
$$

Note that $f_{i j}(t, \vec{k})$ decays exponentially to zero as $t \rightarrow \infty$, uniformly in $\vec{k}$, due to the presence of the projectors in the trace and to the gap condition. Now, the key observation is that

$$
f_{i j}(t, \vec{k})=\partial_{t}^{2} F_{i j}(t, \vec{k}), \quad \text { with } \quad F_{i j}(t, \vec{k})=\operatorname{Tr}\left\{e^{t \widetilde{H}^{(0)}(\vec{k})} \widetilde{P}_{-}(\vec{k}) \partial_{k_{i}} \widetilde{P}_{-}(\vec{k}) e^{-t \widetilde{H}^{(0)}(\vec{k})} \partial_{k_{j}} \widetilde{P}_{-}(\vec{k})\right\}
$$

and $F_{i j}(t, \vec{k})$ decays exponentially to zero as $t \rightarrow \infty$, uniformly in $\vec{k}$. Let us first show how this identity implies (A.1), and let us then come back to its proof. In light of (A.9), we can rewrite

$$
\Sigma_{i j}(\vec{k})=\int_{0}^{\infty} d t t \partial_{t}^{2} F_{i j}(t, \vec{k})=F_{i j}(0, \vec{k})=\operatorname{Tr}\left\{\widetilde{P}_{-}(\vec{k}) \partial_{k_{i}} \widetilde{P}_{-}(\vec{k}) \partial_{k_{j}} \widetilde{P}_{-}(\vec{k})\right\}
$$

Plugging this back into (A.7), we find that $\bar{\sigma}_{i j}(0)$ is equal to the same expression as (A.1), with $P_{-}(\vec{k})$ replaced by $\widetilde{P}_{-}(\vec{k})$. In order to see that we can drop the tilde, note that

$$
\partial_{k_{i}} \widetilde{P}_{-}(\vec{k})=U(\vec{k}) \partial_{k_{i}} P_{-}(\vec{k}) U(\vec{k})^{\dagger}+U(\vec{k})\left[A_{i}, P_{-}(\vec{k})\right] U(\vec{k})^{\dagger}
$$


where $A_{i}=U(\vec{k})^{\dagger} \partial_{k_{i}} U(\vec{k})=i \operatorname{diag}\left(\left(\vec{r}_{1}\right)_{i}, \cdots,\left(\vec{r}_{\mid I}\right)_{i}\right)$. Using this formula, we find that

$$
\operatorname{Tr} \widetilde{P}_{-}(\vec{k})\left[\partial_{k_{i}} \widetilde{P}_{-}(\vec{k}), \partial_{k_{j}} \widetilde{P}_{-}(\vec{k})\right]=\operatorname{Tr} P_{-}(\vec{k})\left[\partial_{k_{i}} P_{-}(\vec{k}), \partial_{k_{j}} P_{-}(\vec{k})\right]+\text { total derivative }
$$

so that the integral over the Brillouin zone of the left side is the same as the integral of $\operatorname{Tr} P_{-}(\vec{k})\left[\partial_{k_{i}} P_{-}(\vec{k}), \partial_{k_{j}} P_{-}(\vec{k})\right]$, and we thus get (A.1).

We are left with proving (A.9) and that $F_{i j}(t, \vec{k})$ decays to zero as $t \rightarrow \infty$. For this purpose, we rewrite (dropping for notational simplicity the arguments of $\widetilde{H}^{(0)}$ and $\left.\widetilde{P}_{ \pm}\right)$):

$$
\partial_{k_{i}} \widetilde{H}^{(0)}=\sum_{\alpha= \pm}\left(\partial_{k_{i}} \widetilde{P}_{\alpha} \widetilde{H}^{(0)} \widetilde{P}_{\alpha}+\widetilde{P}_{\alpha} \partial_{k_{i}} \widetilde{H}^{(0)} \widetilde{P}_{\alpha}+\widetilde{P}_{\alpha} \widetilde{H}^{(0)} \partial_{k_{i}} \widetilde{P}_{\alpha}\right) .
$$

Plugging this identity into (A.8) we find

$$
\begin{aligned}
f_{i j}(t, \vec{k})= & \operatorname{Tr}\left\{e^{t \widetilde{H}^{(0)}} \widetilde{P}_{-}\left(\partial_{k_{i}} \widetilde{P}_{+} \widetilde{H}^{(0)}+\widetilde{H}^{(0)} \partial_{k_{i}} \widetilde{P}_{-}\right) e^{-t \widetilde{H}^{(0)}} \widetilde{P}_{+}\left(\partial_{k_{j}} \widetilde{P}_{-} \widetilde{H}^{(0)}+\widetilde{H}^{(0)} \partial_{k_{j}} \widetilde{P}_{+}\right)\right\} \\
= & -\operatorname{Tr}\left\{\partial_{t} e^{t \widetilde{H}^{(0)}} \widetilde{P}_{-} \partial_{k_{i}} \widetilde{P}_{+} \partial_{t} e^{-t \widetilde{H}^{(0)}} \widetilde{P}_{+} \partial_{k_{j}} \widetilde{P}_{-}\right\}+\operatorname{Tr}\left\{e^{t \widetilde{H}^{(0)}} \widetilde{P}_{-} \partial_{k_{i}} \widetilde{P}_{+} \partial_{t}^{2} e^{-t \widetilde{H}^{(0)}} \widetilde{P}_{+} \partial_{k_{j}} \widetilde{P}_{+}\right\} \\
& +\operatorname{Tr}\left\{\partial_{t}^{2} e^{t \widetilde{H}^{(0)}} \widetilde{P}_{-} \partial_{k_{i}} \widetilde{P}_{-} e^{-t \widetilde{H}^{(0)}} \widetilde{P}_{+} \partial_{k_{j}} \widetilde{P}_{-}\right\}-\operatorname{Tr}\left\{\partial_{t} e^{t \widetilde{H}^{(0)}} \widetilde{P}_{-} \partial_{k_{i}} \widetilde{P}_{-} \partial_{t} e^{-t \widetilde{H}^{(0)}} \widetilde{P}_{+} \partial_{k_{j}} \widetilde{P}_{+}\right\} .
\end{aligned}
$$

Using the fact that $\partial_{k_{i}} \widetilde{P}_{+}=-\partial_{k_{i}} \widetilde{P}_{-}$, this is easily recognized to be equal to

$$
f_{i j}(t, \vec{k})=\partial_{t}^{2} \operatorname{Tr}\left\{e^{t \widetilde{H}^{(0)}} \widetilde{P}_{-} \partial_{k_{i}} \widetilde{P}_{-} e^{-t \widetilde{H}^{(0)}} \widetilde{P}_{+} \partial_{k_{j}} \widetilde{P}_{-}\right\}
$$

which is the same as (A.9), simply because $\left(\partial_{k_{i}} \widetilde{P}_{-}\right) \widetilde{P}_{+}=\widetilde{P}_{-} \partial_{k_{i}} \widetilde{P}_{-}$. Note that, writing $F_{i j}(t, \vec{k})=\operatorname{Tr}\left\{e^{t \widetilde{H}^{(0)}} \widetilde{P}_{-} \partial_{k_{i}} \widetilde{P}_{-} e^{-t \widetilde{H}^{(0)}} \widetilde{P}_{+} \partial_{k_{j}} \widetilde{P}_{-}\right\}$, it is apparent that $F_{i j}(t, \vec{k})$ decays exponentially to zero as $t \rightarrow \infty$, thanks to the projectors under the trace sign and to the gap condition.

\section{Appendix B: The Haldane model}

An interesting model that falls into the general class of two-dimensional systems studied in this paper is the interacting version of the Haldane model [37], which describes fermions hopping on the hexagonal lattice, exposed to a suitable external magnetic field. For simplicity, we neglect the spin degrees of freedom. Let $\Lambda_{L}$ be the triangular lattice, generated by the basis vectors

$$
\vec{\ell}_{1}=\frac{1}{2}(3,-\sqrt{3}), \quad \vec{\ell}_{2}=\frac{1}{2}(3, \sqrt{3}) .
$$

The reciprocal lattice $\Lambda_{L}^{*}$ of $\Lambda_{L}$ is the triangular lattice generated by the vectors

$$
\vec{G}_{1}=\frac{2 \pi}{3}(1,-\sqrt{3}), \quad \vec{G}_{2}=\frac{2 \pi}{3}(1, \sqrt{3}) .
$$

The hexagonal lattice where the electrons hop on can be thought of as the union of two translates of $\Lambda_{L}$, denoted by $\Lambda_{L}^{(A)} \equiv \Lambda_{L}$ and $\Lambda_{L}^{(B)}=\Lambda_{L}+(1,0)$. The creation and annihilation operators associated with the sites of $\Lambda_{L}^{(A)} \cup \Lambda_{L}^{(B)}$ are denoted by $\psi_{\vec{x}, \sigma}^{ \pm}$, with $\vec{x} \in \Lambda_{L}$ and 
$\sigma \in\{A, B\} \equiv I$ : the operators $\psi_{\vec{x}, A}^{ \pm}$create or annihilate a particle at $\vec{x} \in \Lambda_{L}^{(A)} \equiv \Lambda_{L}$, while $\psi_{\vec{x}, B}^{ \pm}$create or annihilate a particle at $\vec{x}+(1,0) \in \Lambda_{L}^{(B)}$. In the notation of section 2 , this corresponds to choosing the displacement vectors as $\vec{r}_{A}=\overrightarrow{0}$ and $\vec{r}_{B}=(1,0)$.

The interacting Haldane model is described by the Hamiltonian (2.7), where the noninteracting part is

$$
\begin{aligned}
\mathcal{H}_{L}^{(0)}= & -t_{1} \sum_{\vec{x} \in \Lambda_{L}}\left[\psi_{\vec{x}, A}^{+} \psi_{\vec{x}, B}^{-}+\psi_{\vec{x}, A}^{+} \psi_{\vec{x}-\vec{\ell}_{1}, B}^{-}+\psi_{\vec{x}, A}^{+} \psi_{\vec{x}-\vec{\ell}_{2}, B}^{-}+\text {h.c. }\right] \\
& -t_{2} \sum_{\vec{x} \in \Lambda_{L}} \sum_{\substack{\alpha= \pm j=1,2,3}}\left[e^{i \alpha \phi} \psi_{\vec{x}, A}^{+} \psi_{\vec{x}+\alpha \vec{\gamma}_{j}, A}^{-}+e^{-i \alpha \phi} \psi_{\vec{x}, B}^{+} \psi_{\vec{x}+\alpha \overrightarrow{\gamma_{j}}, B}^{-}\right] \\
& +W \sum_{\vec{x} \in \Lambda_{L}}\left[\psi_{\vec{x}, A}^{+} \psi_{\vec{x}, A}^{-}-\psi_{\vec{x}, B}^{+} \psi_{\vec{x}, B}^{-}\right],
\end{aligned}
$$

where $\vec{\gamma}_{1}=\vec{\ell}_{1}-\vec{\ell}_{2}, \vec{\gamma}_{2}=\vec{\ell}_{2}, \vec{\gamma}_{3}=-\vec{\ell}_{1}$. See Fig.4.

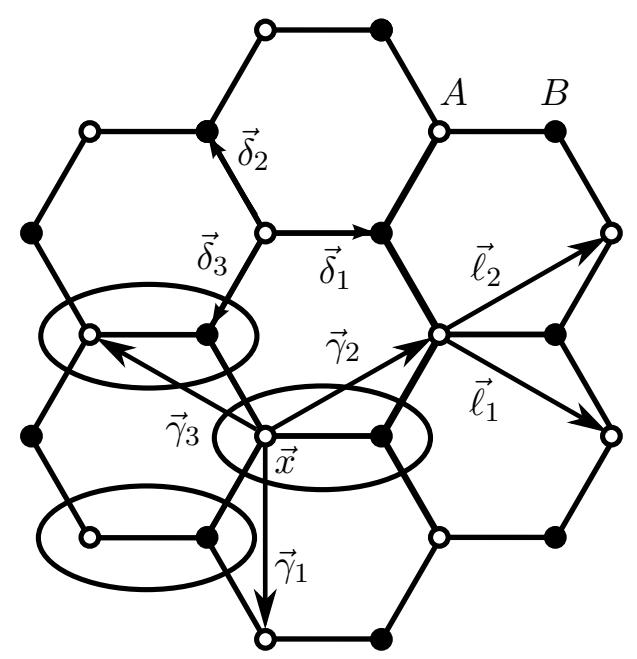

FIG. 4. The honeycomb lattice of the Haldane model. The empty dots belong to $\Lambda_{L}^{(A)}$, while the black dots belong to $\Lambda_{L}^{(B)}$. The ovals encircle the two sites of the fundamental cell, labeled by the position of the empty dot, i.e., of the site of the $A$ sublattice. The two pairs of creation and annihilation operators associated with the two sites of the fundamental cell $\vec{x}$ are denoted by $\psi_{\vec{x}, A}^{ \pm}$ and $\psi_{\vec{x}, B}^{ \pm}$. The nearest neighbor vectors $\vec{\delta}_{i}$, with $\vec{\delta}_{1}=\vec{r}_{B}, \vec{\delta}_{2}=\vec{r}_{B}-\vec{\ell}_{1}$ and $\vec{\delta}_{3}=\vec{r}_{B}-\vec{\ell}_{2}$ are shown explicitly, together with the next-to-nearest neighbor vectors $\vec{\gamma}_{i}$, and the two basis vectors $\vec{\ell}_{1,2}$ of $\Lambda_{L}$.

For definiteness, we assume that $t_{1}>0$ and $t_{2}>0$. The term proportional to $t_{1}$ describes nearest neighbor hopping on the hexagonal lattice. The term proportional to $t_{2}$ describes next-to-nearest neighbor hopping, with the complex phases $e^{ \pm i \phi}$ modeling the effect of an external magnetic field, orthogonal to the plane of the sample, with zero net flux through the hexagonal cell. Finally, the term proportional to $W$ describes a staggered potential, 
favoring the occupancy of the $A$ or $B$ sublattice, depending on whether $W$ is negative or positive.

Let us compute explicitly the Bloch Hamiltonian and the Bloch bands associated with $\mathcal{H}_{L}^{(0)}$ : the Bloch Hamiltonian is

$$
\hat{H}^{(0)}(\vec{k})=\left(\begin{array}{cc}
-2 t_{2} \cos \phi \alpha_{1}(\vec{k})+m(\vec{k}) & -t_{1} \Omega^{*}(\vec{k}) \\
-t_{1} \Omega(\vec{k}) & -2 t_{2} \cos \phi \alpha_{1}(\vec{k})-m(\vec{k})
\end{array}\right)
$$

with

$$
\begin{aligned}
& \alpha_{1}(\vec{k})=\sum_{j=1,2,3} \cos \left(\vec{k} \cdot \vec{\gamma}_{i}\right), \quad \alpha_{2}(\vec{k})=\sum_{j=1,2,3} \sin \left(\vec{k} \cdot \vec{\gamma}_{i}\right), \\
& m(\vec{k})=W-2 t_{2} \sin \phi \alpha_{2}(\vec{k}), \quad \Omega(\vec{k})=1+e^{-i \vec{k} \cdot \vec{\ell}_{1}}+e^{-i \vec{k} \cdot \vec{\ell}_{2}} .
\end{aligned}
$$

The corresponding energy bands are

$$
\varepsilon_{ \pm}(\vec{k})=-2 t_{2} \cos \phi \alpha_{1}(\vec{k}) \pm \sqrt{m(\vec{k})^{2}+t_{1}^{2}|\Omega(\vec{k})|^{2}}
$$

To make sure that the energy bands do not overlap, we assume that $t_{2} / t_{1}<1 / 3$. The two bands can only touch at the Fermi points $\vec{k}_{F}^{ \pm}=\left(\frac{2 \pi}{3}, \pm \frac{2 \pi}{3 \sqrt{3}}\right)$, which are the two zeros of $\Omega(\vec{k})$. The condition that the two bands touch indeed at $\vec{k}_{F}^{\omega}$, with $\omega \in\{+,-\}$, is that $m_{\omega}=0$, with

$$
m_{\omega}:=m\left(\vec{k}_{F}^{\omega}\right)=W+\omega 3 \sqrt{3} t_{2} \sin \phi .
$$

The critical line of the non-interacting Haldane model (i.e., the line in parameter space where the model becomes massless) is then $\left\{(\phi, W): W= \pm 3 \sqrt{3} t_{2} \sin \phi\right\}$. The complement of the critical line consists of four disconnected regions in the $(\phi, W)$ plane, denoted by $\mathcal{R}_{1}=$ $\left\{m_{+}, m_{-}>0\right\}, \mathcal{R}_{2}=\left\{m_{+}>0>m_{-}\right\}, \mathcal{R}_{3}=\left\{m_{+}<0<m_{-}\right\}$, and $\mathcal{R}_{4}=\left\{m_{+}, m_{-}<0\right\}$.

Our theorem applies in the complement of the critical line, non-uniformly in the distance from it. It tells us that, if the Fermi level is chosen inside the gap and $U$ is small enough, then the interacting conductivity is equal to the non-interacting one, which is [37, 42]

$$
\bar{\sigma}_{11}(0)=\bar{\sigma}_{22}(0)=0, \quad \bar{\sigma}_{12}(0)=-\bar{\sigma}_{21}(0)=\frac{1}{4 \pi}\left[\operatorname{sign}\left(m_{-}\right)-\operatorname{sign}\left(m_{+}\right)\right] .
$$

For completeness, let us derive this formula. The starting point is (A.1), which immediately implies the vanishing of the longitudinal conductivity. In order to compute $\bar{\sigma}_{12}(0)$ from (A.1) we need an expression for the projector $P_{-}(\vec{k})$, which can be computed from the Bloch function $u_{-}$:

$$
u_{-}(\vec{k})=\frac{1}{N(\vec{k})}\left(\begin{array}{c}
\sqrt{m(\vec{k})^{2}+t_{1}^{2}|\Omega(\vec{k})|^{2}}-m(\vec{k}) \\
t_{1} \Omega(\vec{k})
\end{array}\right)
$$

where $N(\vec{k})=\left[2 \sqrt{m(\vec{k})^{2}+t_{1}|\Omega(\vec{k})|^{2}}\left(\sqrt{m(\vec{k})^{2}+t_{1}^{2}|\Omega(\vec{k})|^{2}}-m(\vec{k})\right)\right]^{1 / 2}$. The Bloch functions are defined only up to a phase. For instance, another possible choice for the Bloch 
function of the negative band is

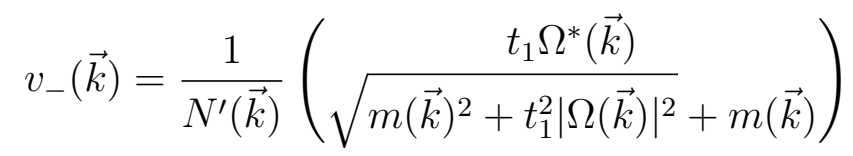

with $N^{\prime}(\vec{k})=\left[2 \sqrt{m(\vec{k})^{2}+t_{1}|\Omega(\vec{k})|^{2}}\left(\sqrt{m(\vec{k})^{2}+t_{1}^{2}|\Omega(\vec{k})|^{2}}+m(\vec{k})\right)\right]^{1 / 2}$. The two functions are related by a phase, namely, $v_{-}(\vec{k})=\frac{\Omega^{*}(\vec{k})}{|\Omega(\vec{k})|} u_{-}(\vec{k}) \equiv e^{i \eta(\vec{k})} u_{-}(\vec{k})$. Note that, if $(\phi, W) \in \mathcal{R}_{1}$ (resp. $(\phi, W) \in \mathcal{R}_{4}$ ), then $v_{-}$(resp. $u_{-}$) is real analytic in $\vec{k}$ over the whole Brillouin zone $\mathcal{B}$. If $(\phi, W) \in \mathcal{R}_{2}$ (resp. $\left.(\phi, W) \in \mathcal{R}_{3}\right)$, then neither $u_{-}$nor $v_{-}$are analytic over the whole $\mathcal{B}$ : $u_{-}$is singular at $\vec{k}_{F}^{+}$(resp. $\vec{k}_{F}^{-}$) and $v_{-}$is singular at $\vec{k}_{F}^{-}$(resp. $\vec{k}_{F}^{+}$). Of course, in any of the regions $\mathcal{R}_{i}$, the projector $P_{-}(\vec{k})$ is independent of the specific choice of the Bloch function, and is analytic over the whole $\mathcal{B}$.

If $(\phi, W) \in \mathcal{R}_{1}$, recalling that $v_{-}$is analytic over the whole Brillouin zone, we write $P_{-}=\left|v_{-}\right\rangle\left\langle v_{-}\right|$, and we thus find

$$
\operatorname{Tr} P_{-}(\vec{k})\left[\partial_{k_{1}} P_{-}(\vec{k}), \partial_{k_{2}} P_{-}(\vec{k})\right]=\left\langle\partial_{k_{1}} v_{-}(\vec{k}), \partial_{k_{2}} v_{-}(\vec{k})\right\rangle-\left\langle\partial_{k_{2}} v_{-}(\vec{k}), \partial_{k_{1}} v_{-}(\vec{k})\right\rangle
$$

Integrating over $\mathcal{B}$ we get zero, which proves that $\bar{\sigma}_{12}(0)=0$, for all $(\phi, W) \in \mathcal{R}_{1}$. The same argument, with $v_{-}$replaced by $u_{-}$, shows that $\bar{\sigma}_{12}(0)=0$, for all $(\phi, W) \in \mathcal{R}_{4}$.

If $(\phi, W) \in \mathcal{R}_{2}$, recalling that $u_{-}$is singular at $\vec{k}_{F}^{+}$and $v_{-}$is singular at $\vec{k}_{F}^{-}$, we write: $P_{-}=\left|v_{-}\right\rangle\left\langle v_{-}\right|$, if $\vec{k} \in \mathcal{B}_{+}$, and $P_{-}=\left|u_{-}\right\rangle\left\langle u_{-}\right|$, if $\vec{k} \in \mathcal{B}_{-}$, where $\mathcal{B}_{ \pm}=\left\{\vec{k} \in \mathcal{B}:\left\|\vec{k}-\vec{k}_{F}^{ \pm}\right\|<\right.$ $\left.\left\|\vec{k}-\vec{k}_{F}^{\mp}\right\|\right\}$, and $\|\vec{q}\|=\min _{n_{1}, n_{2}}\left|\vec{q}+n_{1} \vec{G}_{1}+n_{2} \vec{G}_{2}\right|$ is the norm on the torus $\mathcal{B}$. Note that $\mathcal{B}=\overline{\left(\mathcal{B}_{+} \cup \mathcal{B}_{-}\right)}$. We thus get

$$
\begin{aligned}
\bar{\sigma}_{12}(0)= & i\left[\int_{\mathcal{B}_{+}} \frac{d \vec{k}}{(2 \pi)^{2}}\left(\left\langle\partial_{k_{1}} v_{-}(\vec{k}), \partial_{k_{2}} v_{-}(\vec{k})\right\rangle-\left\langle\partial_{k_{2}} v_{-}(\vec{k}), \partial_{k_{1}} v_{-}(\vec{k})\right\rangle\right)\right. \\
& \left.+\int_{\mathcal{B}_{-}} \frac{d \vec{k}}{(2 \pi)^{2}}\left(\left\langle\partial_{k_{1}} u_{-}(\vec{k}), \partial_{k_{2}} u_{-}(\vec{k})\right\rangle-\left\langle\partial_{k_{2}} u_{-}(\vec{k}), \partial_{k_{1}} u_{-}(\vec{k})\right\rangle\right)\right] .
\end{aligned}
$$

By Stokes' theorem, this can be re-expressed as

$$
\bar{\sigma}_{12}(0)=\frac{1}{(2 \pi)^{2}} \oint_{\partial \mathcal{B}_{+}}[\overrightarrow{\mathcal{V}}(\vec{k})-\overrightarrow{\mathcal{U}}(\vec{k})] \cdot d \vec{k},
$$

where the integration path is run counterclockwise. Moreover, $\overrightarrow{\mathcal{V}}(\vec{k})=\left\langle v_{-}(\vec{k}), i \nabla_{\vec{k}} v_{-}(\vec{k})\right\rangle$ and $\overrightarrow{\mathcal{U}}(\vec{k})=\left\langle u_{-}(\vec{k}), i \nabla_{\vec{k}} u_{-}(\vec{k})\right\rangle$ are the Berry connections of $v_{-}$and $u_{-}$, respectively. Recalling that $v_{-}=e^{i \eta} u_{-}$(see the lines after (B.10)), we get

$$
\bar{\sigma}_{12}(0)=-\frac{1}{(2 \pi)^{2}} \int_{\partial B_{+}} \nabla_{\vec{k}} \eta(\vec{k}) \cdot d \vec{k}=-\frac{1}{2 \pi},
$$

which is the same as (B.8). The same argument, with $v_{-}$replaced by $u_{-}$, shows that $\bar{\sigma}_{12}(0)=1 /(2 \pi)$, for all $(\phi, W) \in \mathcal{R}_{3}$. 


\section{Appendix C: Infinite volume dynamics}

In this appendix, we prove the existence of the thermodynamic and zero temperature limits of real-time correlations, as stated in section 6 , see in particular the discussion after (6.10). The proof is a simple adaptation of [19, 49], the only difference being the choice of boundary conditions (periodic, rather than free). We consider two bounded operators $A, B$ on the fermionic Fock space, even in the fermionic operators, with supports $X$ and $Y$, respectively, independent of $L$. We shall think the torus $\Lambda_{L}$ as a subset of $\Lambda$ 'centered' at the barycenter of $X$ and $Y$, to be denoted $\vec{z}_{0}$. In this way, the 'boundary' of $\Lambda_{L}$ is more and more far from $X$ and $Y$ as $L \rightarrow \infty$. Periodic boundary conditions are enforced by properly choosing (in an $L$-dependent way), the local potentials contributing to $\mathcal{H}_{L}-\mu \mathcal{N}_{L}$, see section $2 \mathrm{~B}$. For notational convenience, we rename these potentials $\Phi_{X}^{L}$, via the following: $\mathcal{H}_{L}-\mu \mathcal{N}=\sum_{X \subset \Lambda_{L}} \Phi_{X}^{L}$. We also drop the vector symbol from the elements of $\Lambda$. For any fixed $X$ (at a fixed distance from the barycenter $z_{0}$ ) and for $L \geq R^{\prime}$,

$$
\left\|\Phi_{X}^{L}-\Phi_{X}^{R^{\prime}}\right\| \rightarrow 0 \quad \text { as } R^{\prime} \rightarrow \infty .
$$

The main result of this appendix is that the following limit exists:

$$
\lim _{\beta \rightarrow \infty} \lim _{L \rightarrow \infty}\left\langle A_{L}(i t) B(0)\right\rangle_{\beta, L}
$$

for all $t \in \mathbb{R}$ and with $A(i t)=e^{i \mathcal{H}_{L} t} A e^{-i \mathcal{H}_{L} t}$.

It is convenient to introduce the following norm:

$$
\left\|\Phi^{L}\right\|_{M, \Lambda_{L}}:=\sup _{x, y \in \Lambda_{L}} \sum_{\substack{X \ni x, y \\ X \subset \Lambda_{L}}} \frac{\left\|\Phi_{X}^{L}\right\|}{F_{M}\left(d_{L}(x, y)\right)}, \quad F_{M}\left(d_{L}(x, y)\right):=\frac{1}{\left(1+d_{L}(x, y)\right)^{M}},
$$

with $d_{L}$ the distance on the torus $\Lambda_{L}: d_{L}(x, y):=\inf _{n \in \Lambda}|x-y+n L|$. Notice that, by the assumptions on $\mathcal{H}_{L}$, the potentials satisfy the bound $\left\|\Phi^{L}\right\|_{M, \Lambda_{L}} \leq C_{M}$, for all $M \geq 0$ and suitable $C_{M}>0$, independent of $L$.

Let $L \geq R^{\prime} \geq R$. In order to prove (C.2), we rewrite

$$
\begin{aligned}
\left\langle A_{L}(i t) B(0)\right\rangle_{\beta, L} \equiv & \left\langle A_{L, L}(i t) B(0)\right\rangle_{\beta, L} \\
= & \left\langle A_{R^{\prime}, R}(i t) B(0)\right\rangle_{\beta, L}+\left\langle\left(A_{L, L}(i t)-A_{L, R}(i t)\right) B(0)\right\rangle_{\beta, L} \\
& +\left\langle\left(A_{L, R}(i t)-A_{R^{\prime}, R}(i t)\right) B(0)\right\rangle_{\beta, L},
\end{aligned}
$$

where $A_{L, R}(i t)$ is the operator evolved with the dynamics generated by

$$
\mathcal{H}_{L, R}:=\sum_{Z \subset \Lambda_{R}} \Phi_{Z}^{L},
$$

that is,

$$
A_{L, R}(i t):=e^{i \mathcal{H}_{L, R} t} A e^{-i \mathcal{H}_{L, R} t} .
$$


By [49, Eq.(2.28)],

$$
\left\|A_{L, L}(i t)-A_{L, R}(i t)\right\| \leq \sup _{x \in X} \sum_{y \in \Lambda_{L} \backslash \Lambda_{R}} \frac{C_{X, M}(t)}{\left(1+d_{L}(x, y)\right)^{M}}, \quad \forall M \in \mathbb{N},
$$

with $C_{X, M}(t)$ independent of $L$ (exponentially growing with $t$, as $t \rightarrow \infty$ ). In particular,

$$
\left\|A_{L, L}(i t)-A_{L, R}(i t)\right\| \leq \varepsilon(R), \quad \text { for some } \varepsilon(R) \rightarrow 0 \text { as } R \rightarrow \infty .
$$

Moreover, the difference $A_{L, R}(i t)-A_{R^{\prime}, R}(i t)$ can be bounded as follows:

$$
\begin{aligned}
\left\|A_{L, R}(i t)-A_{R^{\prime}, R}(i t)\right\| & =\left\|A-e^{-i \mathcal{H}_{L, R} t} e^{i \mathcal{H}_{R^{\prime}, R} t} A e^{-i \mathcal{H}_{R^{\prime}, R} t} e^{i \mathcal{H}_{L, R} t}\right\| \\
& \leq \int_{0}^{t} d s\left\|\left[\mathcal{H}_{L, R}-\mathcal{H}_{R^{\prime}, R}, e^{i \mathcal{H}_{R^{\prime}, R^{s}}} A e^{-i \mathcal{H}_{R^{\prime}, R^{s}}}\right]\right\| \\
& \leq \int_{0}^{t} d s \sum_{Z \subset \Lambda_{R}}\left\|\left[\Phi_{Z}^{L}-\Phi_{Z}^{R^{\prime}}, A_{R^{\prime}, R}(i s)\right]\right\| \\
& \leq \int_{0}^{t} d s C(s)\|A\| \sum_{Z \subset \Lambda_{R}}\left\|\Phi_{Z}^{L}-\Phi_{Z}^{R^{\prime}}\right\| \sum_{w \in Z} \sum_{x \in X} F_{M}\left(d_{R^{\prime}}(w, x)\right) .
\end{aligned}
$$

where in the last step we used the Lieb-Robinson bound (see [49, Theorem 2.1], or [19, Theorem 3.1]). We now use $\sum_{Z \subset \Lambda_{R}} \sum_{w \in Z} \cdots \leq \sum_{z, w \in \Lambda_{R}} \sum_{\Lambda_{R} \supset Z \ni z, w} \cdots$, so that

$$
\begin{aligned}
& \left\|A_{L, R}(i t)-A_{R^{\prime}, R}(i t)\right\| \leq \\
& \quad \leq C^{\prime}(t) \sum_{z \in \Lambda_{R}} \sum_{w \in \Lambda_{R}} F_{M}\left(d_{R^{\prime}}(z, w)\right)\left[\sup _{z, w \in \Lambda_{R}} \frac{\sum_{\Lambda_{R} \supset Z \ni z, w}\left\|\Phi_{Z}^{L}-\Phi_{Z}^{R^{\prime}}\right\|}{F_{M}\left(d_{R^{\prime}}(z, w)\right)}\right] \sum_{x \in X} F_{M}\left(d_{R^{\prime}}(w, x)\right) .
\end{aligned}
$$

Now, using (C.1) and the fact that the norm in (C.3) is bounded uniformly in $L$, the sup in square brackets is smaller than a suitable $\varepsilon\left(R, R^{\prime}\right)$, with $\varepsilon\left(R, R^{\prime}\right) \rightarrow 0$ as $R^{\prime} \rightarrow \infty$. Moreover, $\sum_{w \in \Lambda_{R}} F_{M}\left(d_{R^{\prime}}(z, w)\right) F_{M}\left(d_{R^{\prime}}(w, x)\right) \leq$ (const. $) F_{M}\left(d_{R^{\prime}}(z, x)\right)$, so that

$$
\left\|A_{L, R}(i t)-A_{R^{\prime}, R}(i t)\right\| \leq C^{\prime \prime}(t) \sum_{z \in \Lambda_{R}} \sum_{x \in X} F_{M}\left(d_{R^{\prime}}(z, x)\right) \varepsilon\left(R, R^{\prime}\right) \rightarrow 0 \quad \text { as } R^{\prime} \rightarrow \infty .
$$

We now plug (C.8), (C.9) into (C.4), thus getting

$$
\left|\left\langle A_{L}(i t) B(0)\right\rangle_{\beta, L}-\left\langle A_{R^{\prime}, R}(i t) B(0)\right\rangle_{\beta, L}\right| \leq \widetilde{\varepsilon}\left(R, R^{\prime}\right)
$$

with $\widetilde{\varepsilon}\left(R, R^{\prime}\right) \rightarrow 0$ in the limit $R^{\prime} \rightarrow \infty$, then $R \rightarrow \infty$. Also, it is easy to see that the limit $\lim _{\beta, L \rightarrow \infty}\left\langle A_{R^{\prime}, R}(i t) B(0)\right\rangle_{\beta, L}$ exists, for every fixed $R^{\prime}, R$. In fact, using the boundedness of the fermionic operators and the fact that $\left\|\mathcal{H}_{R^{\prime}, R}\right\| \leq C R^{2}$,

$$
A_{R^{\prime}, R}(i t)=\sum_{n \geq 0} \frac{t^{n}}{n !} \operatorname{ad}_{\mathcal{H}_{R^{\prime}, R}}^{n}(A), \quad\left\|\operatorname{ad}_{\mathcal{H}_{R^{\prime}, R}}^{n}(A)\right\| \leq\|A\|(2 C)^{n} R^{2 n},
$$

where $\operatorname{ad}_{\mathcal{H}_{R^{\prime}, R}}^{n}(A)$ is the $n$-fold commutator of $A$ with $\mathcal{H}_{R^{\prime}, R}$, and $C$ is a constant independent of $R^{\prime}, R$. Therefore, the existence of the $\operatorname{limit}_{\lim _{\beta, L \rightarrow \infty}}\left\langle A_{R^{\prime}, R}(i t) B(0)\right\rangle_{\beta, L}=$ : 
$\left\langle A_{R^{\prime}, R}(i t) B(0)\right\rangle$ follows from the existence of the (time-independent) $\operatorname{limit} \lim _{\beta, L \rightarrow \infty}\left\langle\operatorname{ad}_{\mathcal{H}_{R^{\prime}, R}}^{n}(A) B(0)\right\rangle_{\beta, L}$ for all $n$, which can be proved along the lines of the proof in section 5 .

We now let $L \rightarrow \infty$ in (C.10), so that

$$
\begin{aligned}
-\widetilde{\varepsilon}\left(R, R^{\prime}\right) & \leq \liminf _{\beta, L \rightarrow \infty}\left\langle A_{L}(i t) B(0)\right\rangle_{\beta, L}-\left\langle A_{R^{\prime}, R}(i t) B(0)\right\rangle \leq \\
& \leq \limsup _{\beta, L \rightarrow \infty}\left\langle A_{L}(i t) B(0)\right\rangle_{\beta, L}-\left\langle A_{R^{\prime}, R}(i t) B(0)\right\rangle \leq \widetilde{\varepsilon}\left(R, R^{\prime}\right),
\end{aligned}
$$

that is:

$$
\limsup _{\beta, L \rightarrow \infty}\left\langle A_{L}(i t) B(0)\right\rangle_{\beta, L}-\widetilde{\varepsilon}\left(R, R^{\prime}\right) \leq\left\langle A_{R^{\prime}, R}(i t) B(0)\right\rangle \leq \liminf _{\beta, L \rightarrow \infty}\left\langle A_{L}(i t) B(0)\right\rangle_{\beta, L}+\widetilde{\varepsilon}\left(R, R^{\prime}\right) .
$$

Therefore, letting $R^{\prime}, R \rightarrow \infty$, we find that the liminf and limsup coincide, as desired.

[1] A. Agazzi, J.-P. Eckmann and G. M. Graf. The Colored Hofstadter Butterfly for the Honeycomb Lattice. J. Stat. Phys. 156, 417-426 (2014).

[2] M. Aizenman and G. M. Graf. Localization bounds for an electron gas. J. Phys. A: Math. Gen. 316783 (1998).

[3] H. Araki. Mathematical theory of quantum fields. Oxford University Press (1999).

[4] J. E. Avron, R. Seiler and B. Simon. Homotopy and Quantization in Condensed Matter Physics. Phys. Rev. Lett. 51, 51 (1983).

[5] J. Avron and R. Seiler. Why is the Hall Conductance Quantized? in Open Problems in Mathematical Physics, available at http://web.math.princeton.edu/ aizenman/ OpenProblems.iamp

[6] J. E. Avron, R. Seiler and B. Simon. Charge deficiency, charge transport and comparison of dimensions. Comm. Math. Phys. 159, 399-422 (1994).

[7] G. A. Battle and P. Federbush. A note on cluster expansions, tree graph identities, extra $1 / N$ ! factors!!!, Lett. Math. Phys. 8, 55-57 (1984).

[8] J. Bellissard, A. van Els and H. Schulz-Baldes. The Non-Commutative Geometry of the Quantum Hall Effect. J. Math. Phys. 35, 5373 (1994).

[9] G. Benfatto and V. Mastropietro. On the density-density critical indices in interacting Fermi systems. Comm. Math. Phys. 231, 97-134 (2002)

[10] G. Benfatto and V. Mastropietro. Ward identities and chiral anomaly in the Luttinger liquid. Comm. Math. Phys. 258, 609-655 (2005).

[11] G. Benfatto and V. Mastropietro. Universality relations in non-solvable quantum spin chains. J. Stat. Phys. 138, 1084-1108 (2010).

[12] G. Benfatto, P. Falco and V. Mastropietro. Universal relations for non solvable statistical models. Phys.Rev. Lett. 104, 075701 (2010). 
[13] G. Benfatto, P. Falco and V. Mastropietro. Universality of One-Dimensional Fermi Systems, I. Response Functions and Critical Exponents. Comm. Math. Phys. 330, 153-215 (2014); and II. The Luttinger Liquid Structure. Comm. Math. Phys. 330, 217-282 (2014).

[14] G. Benfatto, G. Gallavotti, A. Procacci and B. Scoppola. Beta function and Schwinger functions for a many fermions system in one dimension. Anomaly of the Fermi surface. Comm. Math. Phys. 160, 93-171 (1994).

[15] S. Bieri and J. Fröhlich. Physical principles underlying the quantum Hall effect. Compt. Rend. Phys. 12, 332-346 (2011).

[16] M. Bishop, B. Nachtergaele and A. Young. Spectral gap and edge excitations of $d$-dimensional PVBS models on half-spaces. Preprint 2015, arXiv:1509.07550 [math-ph].

[17] S. Bravyi and M. B. Hastings. A Short Proof of Stability of Topological Order under Local Perturbations. Comm. Math. Phys. 307, 609-627 (2011).

[18] S. Bravyi, M. B. Hastings and S. Michalakis. Topological quantum order: Stability under local perturbations. J. Math. Phys. 51, 093512 (2010).

[19] J. B. Bru and W. A. de S. Pedra. Lieb-Robinson Bounds for Multi-Commutators and Applications to Response Theory. Springer Briefs in Mathematical Physics, in press.

[20] J. B. Bru and W. A. de S. Pedra. Universal Bounds for Large Determinants from NonCommutative Hölder Inequalities in Fermionic Constructive Quantum Field Theory. Preprint mp_arc 16-16.

[21] D. C. Brydges. A short course on cluster expansions. In Phénomènes critiques, systèmes aléatoires, théories de jauge, (Les Houches, 1984), 129-183. North-Holland, Amsterdam (1986).

[22] D. C. Brydges and P. Federbush. A new form of the Mayer expansion in classical statistical mechanics. J. Math. Phys. 19, 2064-2067 (1978).

[23] S. Coleman and B. Hill. No more corrections to the topological mass term in $\mathrm{QED}_{3}$. Phys. Lett. B 159184 (1985).

[24] N. Datta, R. Fernández and J. Fröhlich. Low-temperature phase diagrams of quantum lattice systems. I. Stability for quantum perturbations of classical systems with finitely-many ground states. J. Stat. Phys. 84, 455 (1996).

[25] N. Datta, R. Fernández, J. Fröhlich and L. Rey-Bellet. Low-temperature phase diagrams of quantum lattice systems. II. Convergent perturbation expansions and stability in systems with infinite degeneracy. Helv. Phys. Acta 69, 752 (1996).

[26] J. Fröhlich and T. Kerler. Universality in quantum Hall systems. Nucl. Phys. B 354, 369-417 (1991).

[27] J. Fröhlich and U. M. Studer. Gauge invariance and current algebra in nonrelativistic manybody theory. Rev. Mod. Phys 65, 733 (1993).

[28] J. Fröhlich, U. M. Studer and E. Thiran. Quantum theory of large systems of non-relativistic matter. Les Houches Lectures 1994, Elsevier (1995); arXiv:cond-mat/9508062.

[29] J. Fröhlich and A. Zee. Large scale physics of the quantum Hall fluid. Nucl. Phys. B 364, 
517-540 (1991).

[30] G. Gallavotti. Renormalization group and ultraviolet stability for scalar fields via renormalization group methods. Rev. Mod. Phys. 57, 471-562 (1985).

[31] G. Gallavotti and F. Nicolò Renormalization theory for four dimensional scalar fields, Parts I and II. Comm. Math. Phys. 100, 545-590 (1985) and 101, 471-562 (1985).

[32] G. Gentile and V. Mastropietro. Renormalization group for one-dimensional fermions. A review on mathematical results. Physics Reports 352 (4), 273-437 (2001).

[33] A. Giuliani. The ground state construction of the two-dimensional Hubbard model on the honeycomb lattice, in Quantum Theory from Small to Large Scales, Lecture Notes of the Les Houches Summer School: Volume 95, August 2010.

[34] A. Giuliani and V. Mastropietro. The 2D Hubbard model on the honeycomb lattice. Comm. Math. Phys. 293, 301-346 (2010).

[35] A. Giuliani, V. Mastropietro and M. Porta. Universality of conductivity in interacting graphene. Comm. Math. Phys. 311, 317 - 355 (2012).

[36] A. Giuliani, V. Mastropietro and M. Porta. Absence of interaction corrections in the optical conductivity of graphene. Phys. Rev. B 83, 195401 (2011).

[37] F. D. M. Haldane. Model for a Quantum Hall Effect without Landau Levels: CondensedMatter Realization of the "Parity Anomaly". Phys. Rev. Lett 61, 2015 (1988).

[38] M. B. Hastings and S. Michalakis. Quantization of Hall Conductance for Interacting Electrons on a Torus. Comm. Math. Phys. 334, 433-471 (2015).

[39] D. R. Hofstadter. Energy levels and wavefunctions of Bloch electrons in rational and irrational magnetic fields. Phys. Rev. B 14, 2239-2249 (1976).

[40] K. Ishikawa and T. Matsuyama. Magnetic Field Induced Multi-Component QED3 and Quantum Hall Effect. Z. Phys C 33, 41-45 (1986).

[41] G. Jotzu et al. Experimental realization of the topological Haldane model with ultracold fermions. Nature 515, 237-240 (2014).

[42] H. Katsura and T. Koma. The $\mathbb{Z}_{2}$ Index of Disordered Topological Insulators with Time Reversal Symmetry. arXiv:1508.05485.

[43] K. v. Klitzing G. Dorda and M. Pepper. New Method for High-Accuracy Determination of the Fine-Structure Constant Based on Quantized Hall Resistance. Phys. Rev. Lett. 45, 494 (1980).

[44] R. Kubo. Statistical-mechanical theory of irreversible processes, I. J. Phys. Soc. Japan 12, 570-586 (1957).

[45] E. H. Lieb and D. W. Robinson. The Finite Group Velocity of Quantum Spin Systems. Comm. Math. Phys. 28, 251-257 (1972).

[46] G. D. Mahan. Many-Particle Physics (3rd ed.), Kluwer/Plenum, New York, 2001.

[47] V. Mastropietro. Non-perturbative renormalization. World Scientific (2008).

[48] S. Michalakis and J. P. Zwolak. Stability of Frustration-Free Hamiltonians. Comm. Math. 
Phys. 322, 277-302 (2013).

[49] B. Nachtergaele, Y. Ogata and R. Sims. Propagation of Correlations in Quantum Lattice Systems. J. Stat. Phys. 124, 1-13 (2006).

[50] B. Nachtergaele and R. Sims. Lieb-Robinson Bounds in Quantum Many-Body Physics. Contemporary Mathematics 529, 141-176 (2010).

[51] W. A. de S. Pedra and M. Salmhofer. Determinant Bounds and the Matsubara UV Problem of Many-Fermion Systems. Comm. Math. Phys. 282, 797-818 (2008).

[52] T. Stauber, N. M. R. Peres and A. K. Geim. Optical conductivity of graphene in the visible region of the spectrum. Phys. Rev. B 78, 085432 (2008).

[53] D. J. Thouless, M. Kohmoto, M. P. Nightingale and M. den Nijs. Quantized Hall Conductance in a Two-Dimensional Periodic Potential. Phys. Rev. Lett. 49, 405 (1982).

[54] C. N. Varney, K. Sun, M. Rigol and V. Galitski. Topological phase transitions for interacting finite systems. Phys. Rev. B, 84, 241105 (2011).

[55] X. G. Wen. Chiral Luttinger liquid and the edge excitations in the fractional quantum Hall states. Phys. Rev. B 41, 12838-12844 (1990).

[56] S.-C. Zhang. The Chern-Simons-Landau-Ginzburg theory of the fractional quantum Hall effect. Int. J. Mod. Phys. B 6, 25-58 (1992). 